\title{
A Bayesian Approach to Estimating Background Flows from a Passive Scalar
}

\author{
Justin A. Krometis \\ Dissertation submitted to the Faculty of the \\ Virginia Polytechnic Institute and State University \\ in partial fulfillment of the requirements for the degree of \\ Doctor of Philosophy \\ in \\ Mathematics \\ Jeffrey T. Borggaard, Co-chair \\ Nathan E. Glatt-Holtz, Co-chair \\ Lizette Zietsman \\ Matthias Chung
}

May 1, 2018

Blacksburg, Virginia

Keywords: Bayesian Statistical Inversion, Bayesian Consistency, Markov Chain Monte Carlo (MCMC), Passive Scalars, Fluid Turbulence

Copyright 2018, Justin A. Krometis 


\title{
A Bayesian Approach to Estimating Background Flows from a Passive Scalar
}

\author{
Justin A. Krometis
}

(ABSTRACT)

We consider the statistical inverse problem of estimating a background flow field (e.g., of air or water) from the partial and noisy observation of a passive scalar (e.g., the concentration of a pollutant). Here the unknown is a vector field that is specified by large or infinite number of degrees of freedom. We show that the inverse problem is ill-posed, i.e., there may be many or no background flows that match a given set of observations. We therefore adopt a Bayesian approach, incorporating prior knowledge of background flows and models of the observation error to develop probabilistic estimates of the fluid flow. In doing so, we leverage frameworks developed in recent years for infinite-dimensional Bayesian inference. We provide conditions under which the inference is consistent, i.e., the posterior measure converges to a Dirac measure on the true background flow as the number of observations of the solute concentration grows large. We also define several computationally-efficient algorithms adapted to the problem. One is an adjoint method for computation of the gradient of the log likelihood, a key ingredient in many numerical methods. A second is a particle method that allows direct computation of point observations of the solute concentration, leveraging the structure of the inverse problem to avoid approximation of the full infinite-dimensional scalar field. Finally, we identify two interesting example problems with very different posterior structures, which we use to conduct a large-scale benchmark of the convergence of several Markov Chain Monte Carlo methods that have been developed in recent years for infinite-dimensional settings. 


\title{
A Bayesian Approach to Estimating Background Flows from a Passive Scalar
}

\author{
Justin A. Krometis
}

(GENERAL AUDIENCE ABSTRACT)

We consider the problem of estimating a fluid flow (e.g., of air or water) from partial and noisy observations of the concentration of a solute (e.g., a pollutant) dissolved in the fluid. Because of observational noise, and because there are cases where the fluid flow will not affect the movement of the pollutant, the fluid flow cannot be uniquely determined from the observations. We therefore adopt a statistical (Bayesian) approach, developing probabilistic estimates of the fluid flow using models of observation error and our understanding of the flow before measurements are taken. We provide conditions under which, as the number of observations grows large, the approach is able to identify the fluid flow that generated the observations. We define several efficient algorithms for computing statistics of the fluid flow, one of which involves approximating the movement of individual solute particles to estimate concentrations only where required by the inverse problem. We identify two interesting example problems for which the statistics of the fluid flow are very different. The first case produces an approximately normal distribution. The second example exhibits highly nonGaussian structure, where several different classes of fluid flows match the data very well. We use these examples to test the functionality and efficiency of several numerical (Markov Chain Monte Carlo) methods developed in recent years to compute the solution to similar problems. 


\section{Dedication}

To Leigh-Anne, Anne, and Mom, the three special women who got me here, and to Linus and Leo, the two amazing boys who keep me going 


\section{Acknowledgments}

Any acknowledgements must start with my amazing committee. Jeff and Nathan, my coadvisors, thank you for agreeing to work with me; for your patience with me as we navigated various life, logistical, and mathematical hurdles; for always being willing to go the extra mile (or, more accurately, hour) with me; and for all your assistance and advice in building my career. Lizette, thank you for giving me a chance when we started revamping Vector Geometry all those years ago, for encouraging me when I expressed an interest in pursuing a doctorate, and for all of your advice and support along the way. Thank you Tia for your help with various technical points as the work progressed. I am tremendously grateful to have had you as mentors and, more importantly, as friends.

I owe a special debt of gratitude to Terry Herdman, my supervisor with Advanced Research Computing (ARC), for supporting my efforts to obtain my Ph.D. From the moment that I

proposed the idea to the day of my defense, you have been more patient than I ever could have hoped, and I cannot imagine having been able to complete my degree without your help. Thank you also to my ARC colleagues, especially Bob, Brian, James, and John, who helped me along the way.

Thank you to the many members of the Math Department who made this possible: to Peter for your work in building such supportive department and for your advice and friendship over almost two decades, to Nicole for always being there whenever I had a question, and to the many other members of the department who provided support, advice, and friendship over the years.

This work was supported in part by National Science Foundation grant DMS-1313272 and Simons Foundation grant 515990. I would also like to thank the Mathematical Sciences 
Research Institute, the Tulane University Math Department, and the International Centre for Mathematical Sciences (ICMS) where significant portions of this work were developed and carried out. The Society for Industrial and Applied Mathematics (SIAM) and NSF grant DMS-1613965 supported my attendance of the 2016 Gene Golub SIAM Summer School, where I presented and received feedback on the beginnings of this work. Similarly, NSF grant 1700124 and the Virginia Tech Interdisciplinary Center for Applied Math (ICAM), respectively, provided funds for travel to the 2016 FOMICS Winter School on Uncertainty Quantification and 2017 ICMS workshop Probabilistic Perspectives in Nonlinear Partial Differential Equations, where I learned a lot of important background material and gained insight into ongoing work in related fields.

My advisors and I would like to thank G. Didier, J. Foldes, S. McKinley, C. Pop, G. Richards, G. Simpson, A. Stuart, and J. Whitehead for many useful discussions and thoughtful feedback. We look forward to working with many of you more in the future.

And finally, thank you to my family, without whom this would not have been possible or worth it. Linus, thank you for being interested in my work as it went along, from pointing out the graph that looked like a dragon to listening to my practice defense. Leo, thank you for being an amazing light that entered my life in the middle of this process and for sleeping while I learned Functional Analysis; sorry for the stress about math that I caused you here at the end. Leigh-Anne, thank you for your love, support, and advice throughout this long journey, and for always being there when I needed to travel somewhere or just pull a late night. Hopefully all of this work will at least mean that we get fewer envelopes addressed to "Dr. and Mrs. Krometis." 


\section{Contents}

List of Figures $-x$

List of Tables $\quad$ xiv

1 Introduction $\quad 1$

2 Review of Literature $\quad 5$

2.1 Bayesian Inference and $\mathrm{MCMC} \ldots \ldots \ldots$

2.2 Advection-Diffusion . . . . . . . . . . . . . . . . . . . . . 7

3 Mathematical Framework and Bayesian Inference $\quad 9$

3.1 Representation of Divergence-Free Background Flows . . . . . . . . . . . . . 9

3.2 Mathematical Setting . . . . . . . . . . . . . . . . 11

3.3 Ill-posedness of the Inverse Problem . . . . . . . . . . . . . . . . 15

3.4 Bayesian Inference . . . . . . . . . . . . . . . . . . . 17

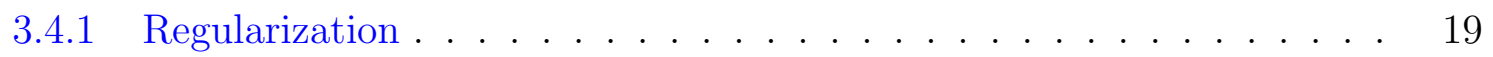

3.4.2 Bayes' Theorem .......................... 20

4 Consistency of the Bayesian Posterior $\quad 23$

4.1 Continuity of Inverse Map . . . . . . . . . . . . . . . . . . . . 24 
4.2 Convergence of Posterior to the True Scalar Field . . . . . . . . . . . . . . . 29

4.3 Convergence of Posterior Measure to the True Vector Field $\mathbf{v}^{\star} \ldots$. . . . . . 41

5 Overview of MCMC Methods 44

5.1 Metropolis-Hastings in Infinite Dimensions . . . . . . . . . . . . . . . . 45

5.2 The Independence Sampler . . . . . . . . . . . . . . . . . . . . . . . . . 52

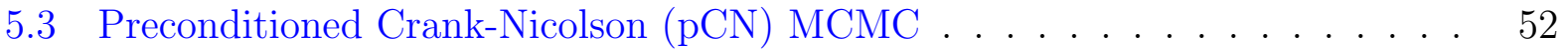

5.4 Metropolis-Adjusted Langevin (MALA) MCMC . . . . . . . . . . . . 54

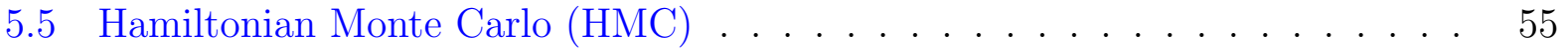

5.6 Summary of MCMC Methods . . . . . . . . . . . . . . . . . 60

6 Practical and Theoretical Implementation Challenges 62

6.1 Evaluation of $\mathcal{G} \ldots \ldots \ldots \ldots$. . . . . . . . . . . . . . . . 62

6.1.1 Computational Challenges ................. 63

6.2 Adjoint Method for Evaluating the Gradient of $\Phi$. . . . . . . . . . . . . 64

7 A Particle Method for Sparse Point Observations $\quad 69$

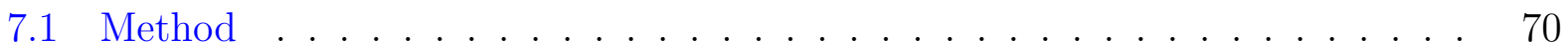

7.2 Parallelization . . . . . . . . . . . . . . . . . . . . . . 71

7.3 Computational Complexity . . . . . . . . . . . . . . . . 72

7.4 Limitations . . . . . . . . . . . . . . . . . . . 75 
8.1 A Prior for Passive Scalar Problems . . . . . . . . . . . . . . . . 77

8.2 Example 1: Single-welled Posterior . . . . . . . . . . . . . . . . 79

8.2.1 Posterior Structure . . . . . . . . . . . . . . . . . . 79

8.2 .2 MCMC Sampling . . . . . . . . . . . . . . . . . . . 83

8.2.3 Convergence of Observables . . . . . . . . . . . . . . 85

8.2.4 Convergence of Measures . . . . . . . . . . . . . . . . . . . . . 87

8.2.5 Equal Runtime Comparison . . . . . . . . . . . . . . . . 91

8.3 Example 2: Multi-welled Posterior . . . . . . . . . . . . . . . . . . . 94

8.3.1 Posterior Structure . . . . . . . . . . . . . . . . . . . 96

8.3.2 Sampling and Selection of Free Parameters . . . . . . . . . . . . . . 97

8.3.3 Convergence of Observables . . . . . . . . . . . . . . . . 103

8.3.4 Convergence of Measures . . . . . . . . . . . . . . . 104

8.3.5 Equal Runtime Comparison . . . . . . . . . . . . . . . 109

8.3.6 Extension to Higher Dimensions via Particle Method . . . . . . . . . 112

8.4 Summary of Results of Numerical Experiments . . . . . . . . . . . . . . 115

9 Conclusions and Future Work $\quad 117$

$\begin{array}{ll}\text { Bibliography } & 119\end{array}$ 


\section{List of Figures}

1.1 Contour plot of $\theta(\mathbf{v})$ for example high-dimensional $\mathbf{v} \ldots \ldots . . . . . .2$

1.2 Histogram of two components of $\mathbf{v}$ for the example problem in Section 8.3. . 4

3.1 Illustration of Consideration 3.13: The addition of noise to perfect measurements $\mathcal{Y}^{\star}$ drives some of the observations $\mathcal{Y}$ outside the range of $\theta_{0}$, violating the maximum principle. . . . . . . . . . . . . . . . . . 17

4.1 Ill-posedness Example \#1: Any $\mathbf{v}=[0, f(x)]$ produces the same $\theta \ldots . . .25$

4.2 Ill-posedness Example \#2: Any multiple of $\mathbf{v}^{\star}$ produces the same $\theta$. . . . . 25

5.1 Particle moving in an example 2D Hamiltonian system (5.26). . . . . . . . . 57

5.2 Diagram of Metroplis-Hastings MCMC . . . . . . . . . . . . . . 61

6.1 Number of Fourier components $\theta_{\mathbf{k}}>0.001$ for small and large $\kappa . \ldots 64$

7.1 Traces of 5 simulated Itô diffusions with the same final position. . . . . . . 70

7.2 GPU thread blocks[67]. . . . . . . . . . . . . . . . . 72

8.1 The Kraichnan energy spectrum $E(k)$ (8.1) in one dimension (compare with Figure 6 from $[18]) \ldots \ldots \ldots \ldots \ldots$

8.2 The Kraichnan energy spectrum $\frac{1}{2 \pi\|\mathbf{k}\|_{2}} E\left(\|\mathbf{k}\|_{2}\right)$ in two dimensions. . . . . . . 78

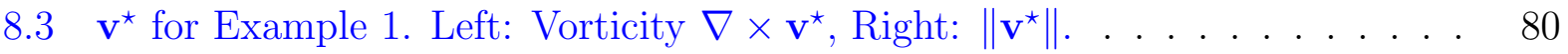


8.4 Prior $\left(\mu_{0}\right)$ and posterior $\left(\mu_{\mathcal{y}}\right)$ marginal distributions for the first eight com-

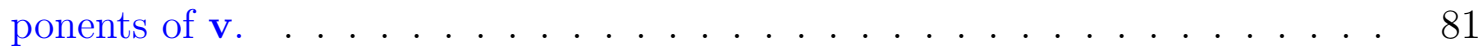

8.5 Posterior two-dimensional histograms for the first eight components of $\mathbf{v}$. . $\quad 82$

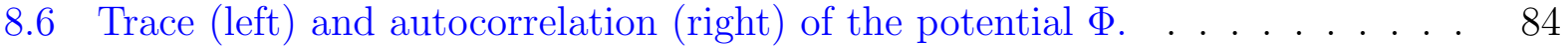

8.7 Relative error for the mean value (cumulative moving average) of observables scalar variance, scalar dissipation rate, enstrophy, and enstrophy dissipation for 10,000 samples, IS (top left), pCN (top right), MALA (bottom left), and HMC (bottom right). . . . . . . . . . . . . . . . . .

8.8 Relative error for the mean value (cumulative moving average) of scalar differences at $t=1$ between the origin and $\left[\frac{1}{2}, \frac{1}{2}\right],\left[\frac{1}{4}, \frac{1}{4}\right],\left[\frac{1}{8}, \frac{1}{8}\right],\left[\frac{1}{16}, \frac{1}{16}\right]$ for 100,000 samples, IS (top left), pCN (top right), MALA (bottom left), and HMC (bottom right). . . . . . . . . . . . . . . . .

8.9 Computed marginal distribution for each of the first eight vector field components for 10,000 samples, IS (top left), pCN (top right), MALA (bottom left), and HMC (bottom right). . . . . . . . . . . . . . . . . .

8.10 Total variation norm between computed and "true" marginal probability density function for $v_{2}, \ldots, v_{9}$ for 10,000 samples, IS (top left), pCN (top right), MALA (bottom left), and HMC (bottom right). . . . . . . . . .

8.11 Relative error for the mean value (cumulative moving average) of observables scalar variance, scalar dissipation rate, enstrophy, and enstrophy dissipation for runtime equivalent to 10,000 HMC samples, IS (top left), pCN (top right), MALA (bottom left), and HMC (bottom right). . . . . . . . . . 
8.12 Total variation norm between computed and "true" marginal probability density function for $v_{2}, \ldots, v_{9}$ for runtime equivalent to 10,000 HMC samples, IS (top left), pCN (top right), MALA (bottom left), and HMC (bottom right). .

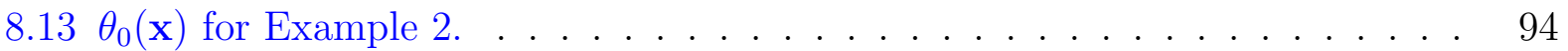

$8.14 \mathbf{v}^{\star}$ for Example 2. . . . . . . . . . . . . . . . . . . . . . . . . . . . 94

8.15 Posterior $\left(\mu_{\mathcal{Y}}\right)$ marginal distributions for the first eight components of $\mathbf{v}$. . 97

8.16 Posterior two-dimensional histograms for the first eight components of $\mathbf{v}$. . 98

8.17 Posterior probability density function for vector field enstrophy $\left(\frac{1}{2}\|\nabla \times \mathbf{v}\|_{L^{2}}^{2}\right) . \quad 99$

8.18 Acceptance ratio by $\mathrm{pCN} \beta \ldots \ldots$. . . . . . . . . . . . . . 100

8.19 Acceptance ratio by MALA h. . . . . . . . . . . . . . . . 100

8.20 Acceptance ratio by HMC $\tau$ and $I=\frac{\tau}{\epsilon} \ldots \ldots \ldots$. . . . . . . . . . . . 101

8.21 Normalized sampling rate by MCMC method (Higher is better, Independence Sampler=1). . . . . . . . . . . . . . . . . . . . . . 101

8.22 Trace (left) and autocorrelation (right) of the potential $\Phi$. . . . . . . . . . 102

8.23 Trace of $v_{2}, \ldots, v_{5}$ by sample number, pCN . . . . . . . . . . . . . 103

8.24 Trace of $v_{2}, \ldots, v_{5}$ by sample number, HMC . . . . . . . . . . . . 103

8.25 Relative error for the mean value of observables scalar variance, scalar dissipation rate, enstrophy, and enstrophy dissipation for 10,000 samples, pCN (left) and HMC (right). . . . . . . . . . . . . . . . . . . 104

8.26 Relative error for scalar differences between various points for 150,000 samples, pCN (left) and HMC (right). . . . . . . . . . . . . . . . . 104 
8.27 Computed marginal distribution for each of the first eight vector field components for 150,000 samples, pCN (left) and HMC (right) . . . . . . . . . . . 105

8.28 Total variation norm between computed (150,000 samples) and "true" marginal probability density function for $v_{2}, \ldots, v_{9}, \mathrm{pCN}$ (left) and HMC (right). . . . 106

8.29 Posterior two-dimensional histograms for the first eight components of $\mathbf{v}, \mathrm{pCN}$ sampler (Single chain) . . . . . . . . . . . . . . . . . 107

8.30 Posterior two-dimensional histograms for the first eight components of $\mathbf{v}$, HMC sampler (Single chain). . . . . . . . . . . . . . . . . . . . . 108

8.31 Trace of $v_{2}, \ldots, v_{5}$ by sample number for runtime equivalent to $150,000 \mathrm{HMC}$ samples, pCN (left) and HMC (right). . . . . . . . . . . . . . . . 109

8.32 Total variation norm between computed and "true" marginal probability density function for $v_{2}, \ldots, v_{9}$ for pCN and HMC, by computational time $(1$ computational unit $=1$ HMC sample) . . . . . . . . . . . . . . . . 110

8.33 Total variation norm between computed and "true" marginal probability density function for $v_{2}, \ldots, v_{9}$ across 100 pCN chains and 1 HMC chain, by computational cost (1 computational unit $=1$ HMC sample on one computer) . . 111

8.34 Posterior marginal distributions for the first eight components of $\mathbf{v}$. . . . . 113

8.35 Posterior two-dimensional histograms for the first eight components of $\mathbf{v} . \quad$. . 114 


\section{List of Tables}

5.1 Comparison of MCMC Methods. . . . . . . . . . . . . . . . . 61

8.1 Problem parameters for Example 1. . . . . . . . . . . . . . 79

8.2 MCMC Parameters Applied to Example 1. . . . . . . . . . . . . . 83

8.3 MCMC Acceptance Rates for Example 1. . . . . . . . . . . . . 83

8.4 Observables. . . . . . . . . . . . . . . . . . 85

8.5 Problem parameters for Example 2. . . . . . . . . . . . . . . 96

8.6 MCMC Parameters Applied to Example 1. . . . . . . . . . . . . . . . 101

8.7 Time to compute $\mathcal{G}$ for 197 basis functions $\left(T_{1}\right)$ vs. 3,209 basis functions $\left(T_{2}\right)$, by Method. . . . . . . . . . . . . . . . . . . . 112 


\section{Chapter 1}

\section{Introduction}

In this work we consider the inverse problem of estimating a background fluid flow from observations of a pollutant or other solute advecting and diffusing within the fluid. The initial condition is assumed to be known, so the problem can be interpreted as a controlled experiment, where the solute (e.g., a dye) is added in known locations and then observed as the system evolves to investigate the structure of the underlying fluid flow.

The physical model considered is the two-dimensional advection-diffusion equation on the $2 \mathrm{D}$ periodic domain $\mathbb{T}^{2}=[0,1]^{2}$ :

$$
\frac{\partial}{\partial t} \theta(t, \mathbf{x})=-\mathbf{v}(\mathbf{x}) \cdot \nabla \theta(t, \mathbf{x})+\kappa \Delta \theta(t, \mathbf{x}) \quad, \quad \theta(0, \mathbf{x})=\theta_{0}(\mathbf{x})
$$

Here

- $\theta: \mathbb{R}^{+} \times \mathbb{T}^{2} \rightarrow \mathbb{R}$ is a passive scalar, typically the concentration of some solute of interest, which is spread by diffusion and by the motion of a (time-stationary) fluid flow $\mathbf{v}$. This solute is "passive" in that it does not affect the motion of the underlying fluid.

- $\mathbf{v}: \mathbb{T}^{2} \rightarrow \mathbb{R}^{2}$ is an incompressible background flow, i.e., $\mathbf{v}$ is constant in time and satisfies $\nabla \cdot \mathbf{v}=0$.

- $\kappa>0$ is the diffusion coefficient, which models the rate at which local concentrations 
of the solute spread out within the solvent in the absence of advection.

We obtain finite observations $\mathcal{Y} \in Y$ (e.g., $\mathbb{R}^{n}$ or $\mathbb{C}^{n}$ ) subject to additive noise $\eta$, i.e.

$$
\mathcal{Y}=\mathcal{G}(\mathbf{v})+\eta \quad, \quad \eta \sim \mathbb{Q}_{0}
$$

for some measure $\mathbb{Q}_{0}$ related to the precision of the observations. Here, $\mathcal{G}: H \rightarrow Y$ is the parameter-to-observable, or forward, map. This $\mathcal{G}$ associates background flow $\mathbf{v}$ sitting in a suitable function space $H$ with a finite collection of measurements (observables) of the resulting $\theta=\theta(\mathbf{v})$. The observations may take a number of forms, such as:

- Point: $\mathcal{Y}_{j}=\theta\left(t_{j}, \mathbf{x}_{j}\right)+\eta_{j}$

- Spectral: $\mathcal{Y}_{j}=\left\langle\theta\left(t_{j}\right), \mathbf{e}_{\mathbf{k}_{j}}\right\rangle_{L^{2}\left(\mathbb{T}^{2}\right)}+\eta_{j}$ for some basis $\left\{\mathbf{e}_{\mathbf{k}}\right\}$

- Other physical quantities of interest from $\theta$, such as variance, dissipation rate, or structure functions

This work will focus on point observations as the most obvious practical implementation.

The behavior of passive scalars has been the subject of considerable research, as for advection-dominated (low- $\kappa$ ) cases $\theta$ can quickly become very complex;[81] see, for example, Figure 1.1. For turbulent flows, passive scalars exhibit regions of plateaus and steep cliffs $[18,78]$ and have been modeled using stochastic vector fields $[50,51]$ that we will use to motivate the selection of some of our parameters in Section 8.1.

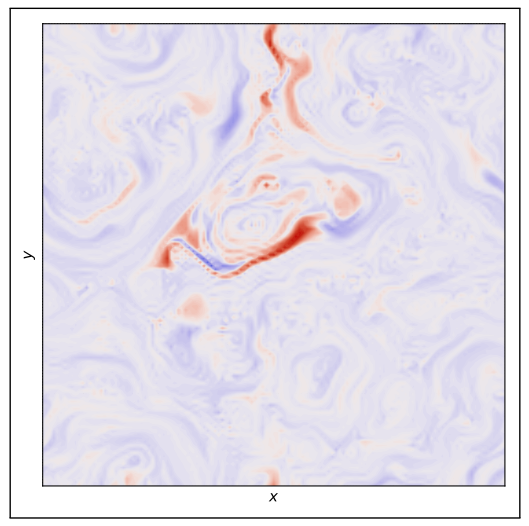

Figure 1.1: Contour plot of $\theta(\mathbf{v})$ for example highdimensional $\mathbf{v}$.

As we illustrate below, the proposed inverse problem is ill-posed, i.e., there may be many 
or no background flows $\mathbf{v}$ that match a given dataset $\mathcal{Y}$. To address this issue, we adopt a Bayesian approach, incorporating prior knowledge of background flows and models of the observation error to develop probabilistic estimates of $\mathbf{v}$. Summaries of the Bayesian approach to inverse problems can be found in [34] and [46]. Moreover, since the target of the inversion, the background flow $\mathbf{v}$, is infinite-dimensional, this work will leverage the considerable amount of recent research in infinite-dimensional Bayesian inference, grounding much of our approach in the overview of the field provided in [24].

In Bayesian inference, the solution to the inverse problem is not a single background flow $\mathbf{v}$ but a probability measure, called the "posterior," on the space of possible background flows. The posterior quantifies our degree of knowledge for $\mathbf{v}$ once we have fixed a description of measurement uncertainty (i.e., the distribution of $\eta$ ) and a "prior" probability measure $\mu_{0}$ on $H$ which reflects knowledge of $\mathbf{v}$ before the incorporation of measurements. The prior may be based on, for example, historical data on or theoretical understanding of the structure of $\mathbf{v}$. To compute observables, such as the mean, variance, or (normalized) histogram of a given quantity on $\mathbf{v}$ or $\theta$, we use Metropolis-Hastings Markov Chain Monte Carlo (MCMC) algorithms that are well-defined in infinite dimensions, in particular those presented in [11] and [21].

The problem setting and methodology reflects several motivations. First, the problem reflects a natural framework to develop and explore statistical estimation techniques applied to solute concentration problems. Second, the setting provides an example that we use to explore the theory underlying the Bayesian framework. Finally, we leverage the application to test, refine, and compare MCMC algorithms recently developed for infinite-dimensional problems. This work makes a number of important contributions. We lay out a Bayesian framework for the estimation of divergence-free background flows. We prove conditions under which the Bayesian inference is consistent, i.e., the noise is averaged out and the posterior measure 
converges uniquely to the true background flow as the number of measurements grows large. We define, prove, and numerically implement an adjoint method for the efficient computation of the gradient of the log likelihood, a key ingredient in higher-order MCMC methods. Furthermore, we introduce a particle method for the computation of point observations of $\theta$ that is both computationally efficient for high-dimensional, sparse-observation applications and easily parallelizable on modern architectures. Finally, we identify two interesting examples for which the resulting posterior measures have very different structures - one fairly simple and one highly complex. For illustration, an example two-component histogram for the latter problem is shown in Figure 1.2. We use these two examples to conduct a systematic, large-scale numerical study to benchmark the convergence of several varieties of MCMC methods for "easy" and "hard" problems.

The structure of the paper is as follows. Chapter 3 describes the mathematical framework of the inverse problem, why it is ill-posed in the traditional sense, and how adopting the Bayesian approach redefines the inverse problem in such a way that the answer is well-defined. In Chapter 4, we prove a theorem on consistency of the Bayesian posterior measure as the number of observations grow large. Chapter 5 describes recently-developed MCMC methods in infinite dimensions. Chapter 6 describes numerical methods for solving the

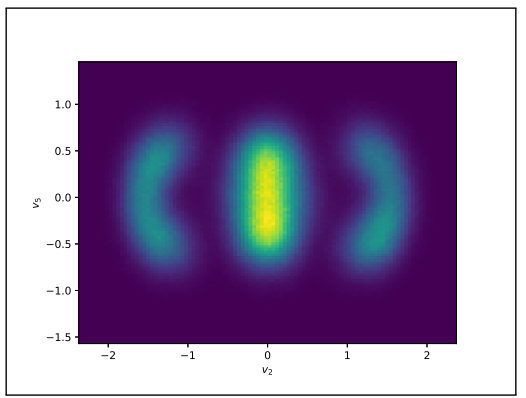

Figure 1.2: Histogram of two components of $\mathbf{v}$ for the example problem in Section 8.3. advection-diffusion equation (1.1) (and some inherent complexities) and defines an adjoint method for computing gradients required for some MCMC methods. Chapter 7 describes a particle method for computation of point observations of $\theta$. Chapter 8 describes the results of the inference as applied to two example problems. Chapter 9 provides a summary of the work and suggests a number of paths for relevant future work. 


\section{Chapter 2}

\section{Review of Literature}

\subsection{Bayesian Inference and MCMC}

Bayesian inference dates to the publication of Bayes' original theorem on conditional and prior probability in 1763.[7] Comprehensive overviews of modern Bayesian techniques, from the basics of probability theory to computational practicalities, can be found in [34] and [46]. The survey article [3] provides a practical summary of how Bayesian inference works as applied to a simple example. The lecture notes [24] summarize recent advances in the theory of Bayesian inference for infinite-dimensional problems (e.g., where the target of the inversion is a function), building on the work in [80]. A number of recent works have explored applications of Bayesian inference to infinite-dimensional problems; to name just two, the paired papers [16] and [69] apply infinite-dimensional Bayesian inference to partial differential equations describing seismic wave propagation and ice sheet flow, respectively.

Consistency of Bayesian estimators has been of interest since at least the 1800s, [53] with rigorous proofs of convergence for some problems appearing in the mid-twentieth century; $[27$, 55] see, e.g., the description in [54]. Section 4.2 and Appendix B of [34] provide a summary of some of the basic ideas and results, including how compactness can be used to extend finitedimensional consistency results to continuous parameter spaces. Rates of convergence to the correct parameter value using upper and lower bounds on the tail and core of the posterior measure, respectively, are provided in [77]. By contrast, there has also been much work 
$[26,33,76]$ describing examples where Bayesian estimators are not consistent, particularly for infinite-dimensional problems. More general results on statistical asymptotics, including cases where Bayesian consistency does and does not hold, are summarized in [54].

Metropolis-Hastings Markov Chain Monte Carlo (MCMC) methods date to the foundational work [60], in which MCMC was used to compute statistical states of a system of molecules. The method was then generalized to more abstract applications in [41]. The application of Langevin diffusions to MCMC methods began to be explored in the 1990s.[37, 72] These methods, generally referred to as Metropolis-adjusted Langevin Algorithms (MALA), incorporate data into the generation of proposal samples, yielding a proposal kernel that is reversible with respect to the target measure. Around the same time, Hamiltonian, or Hybrid, Monte Carlo (HMC) schemes began to be introduced to the statistical community[64], leveraging the use of Hamiltonian dynamics for stochastic problems laid out in [29]. The MALA and HMC methods include parameters that can be tuned to the problem, leading to investigations into the optimal selection of these parameters and the convergence of MCMC methods more generally.[71, 73]

Substantial recent work has gone into extending these MCMC methods to problems in which the space to be sampled is high- or infinite-dimensional, e.g., spaces of functions.[8, 9, 10] The goal of these efforts has been to define kernels that are both well-defined and yield robust convergence even as the number of dimensions to be sampled grows large. The extension of Metropolis-Hastings methods to generalized state spaces was described in [82]. The behavior of the traditional random walk approach as the dimension grows large was investigated in [59] for a large class of target measures. Variations of random walk and MALA algorithms suitable for infinite-dimensional problems were laid out in [21]; optimal choice of the step size parameter in the MALA algorithm was shown in [70]. HMC was similarly extended to infinite-dimensions in [11], work that was later generalized in [12] and 
[36]. Dimension-independent convergence of some of the above methods has been investigated by showing that the kernels have spectral gaps, $[40,83]$ leveraging a generalized version of Harris' Theorem[38, 39] for Markov kernels.

While we did not use them in this work, we note that other approaches exist to quantifying uncertainty. Stochastic finite elements have been used in a number of physical applications with uncertain parameters.[35] The spectral method known as "polynomial chaos" [63, 87, 88] involves expansion of the stochastic quantity in terms of an orthogonal Wiener-Askey chaos. $[4,86]$ This method was leveraged for approximation of Bayesian posterior distributions in $[57]$ and [58].

\subsection{Advection-Diffusion}

To our knowledge, this work is the first to apply Bayesian inference to the problem of estimating a background fluid flow from measurements of a passive scalar. However, a number of works, such as [2], have used inversion techniques to determine a source (forcing) term in advection-diffusion problems. In these problems, the background flow is assumed to be known and the initial condition is assumed to be zero; the goal is to determine the function (in particular the location) from which the pollutant is being dispersed. This source-finding work was extended to ensure robustness to uncertainties in the velocity field in [89]. Another investigation of a similar problem is [30], which used an infotaxis-based search algorithm to determine whether an advecting and diffusing fluid is well-mixed from finite measurements.

A description of the mathematical theory and some numerical approaches for simulating incompressible flows can be found in [56]. Numerical difficulties in modeling the behavior of passive scalars for advection-dominated cases are described in [61] and [81]. Particle methods for simulation of advection-diffusion equations are described in [19] and [25]; these 
approaches motivate the particle method that we introduce in Chapter 7, which also leverages Kolmogorov's backward equation $[48,68]$. The simulation method involves discretization of a stochastic evolution equation as described in, for example, [43] or [47].

While we do not consider the case of time-dependent turbulence in this work, a summary of the phenomena and theory underlying the behavior of passive scalars in turbulent flows is described in [78], [79], and [84]. The quantities of interest on both $\theta$ and $\mathbf{v}$ described therein motivate observables computed in Chapter 8. Passive scalars exhibit similar behavior for turbulent and random flows, so the latter, simpler case may be used to model the former. One such model was introduced by Kraichnan; $[49,50,51]$ we will use the energy spectrum from this model in Section 8.1. The structure of passive scalars for randomly-advected flows is illustrated in the numerical study in [18]. 


\section{Chapter 3}

\section{Mathematical Framework and}

\section{Bayesian Inference}

In this section, we describe the mathematical framework of the inverse problem. We begin by defining the functional analytic setting for the problem, including how we represent divergence-free background flows. We then describe reasons why the inverse problem is illposed, i.e., why a given set of measurements $\mathcal{Y}$ cannot identify a single background flow $\mathbf{v}$ that generated them. We then define the Bayesian approach and describe how it redefines the inverse problem in a way that eliminates the ill-posedness.

\subsection{Representation of Divergence-Free Background Flows}

The target of the inference is a divergence-free background flow $\mathbf{v}$, so we start by describing the space $H$ of such flows that we will consider, and how we will define probability measures on this (infinite-dimensional) space. We begin with a definition of Sobolev spaces on periodic functions, which is taken from [74].

Definition 3.1 (Sobolev Space of Periodic Functions (Robinson, 2001)). The Sobolev space 
of periodic functions $H^{s}$ defined on $\mathbb{T}^{2}=[0,1]^{2}$ is

$$
H^{s}\left(\mathbb{T}^{2}\right)=\left\{u: u=\sum_{\mathbf{k} \in \mathbb{Z}^{2}} c_{\mathbf{k}} e^{2 \pi i \mathbf{k} \cdot \mathbf{x}}, \overline{c_{\mathbf{k}}}=c_{-\mathbf{k}}, \sum_{\mathbf{k} \in \mathbb{Z}^{2}}\|\mathbf{k}\|^{2 s}\left|c_{\mathbf{k}}\right|^{2}<\infty\right\}
$$

We will adapt this definition to our setting of periodic divergence-free background flows by defining the coefficients $c_{\mathbf{k}}$ as

$$
c_{\mathbf{k}}=v_{\mathbf{k}} \frac{\mathbf{k}^{\perp}}{\|\mathbf{k}\|}, \quad \overline{v_{\mathbf{k}}}=-v_{-\mathbf{k}}
$$

where $\mathbf{k}^{\perp}=\left[-k_{y}, k_{x}\right]$ to ensure $\mathbf{k} \cdot \mathbf{k}^{\perp}=0$.

Then we define the Sobolev space of periodic divergence-free background flows that we will use as our problem setting as follows.

Definition 3.2 (Sobolev Space of Periodic Divergence-Free Background Flows, H). The Sobolev space of periodic divergence-free background flows $H$ on $\mathbb{T}^{2}=[0,1]^{2}$ is

$$
H=H^{2+\epsilon}\left(\mathbb{T}^{2}\right)=\left\{\mathbf{v}: \mathbf{v}=\sum_{\mathbf{k} \in \mathbb{Z}^{2}} v_{\mathbf{k}} \frac{\mathbf{k}^{\perp}}{\|\mathbf{k}\|} e^{2 \pi i \mathbf{k} \cdot \mathbf{x}}, \overline{v_{\mathbf{k}}}=-v_{-\mathbf{k}}, \sum_{\mathbf{k} \in \mathbb{Z}^{2}}\|\mathbf{k}\|^{4+2 \epsilon}\left|v_{\mathbf{k}}\right|^{2}<\infty\right\}
$$

The exponent $2+\epsilon$ is chosen so that vector fields in $H$, as well as their corresponding solutions $\theta(\mathbf{v})$, exhibit some important properties of continuity, which are described in Section 3.2.

For brevity, we also write the expansion of $\mathbf{v} \in H$ as simply

$$
\mathbf{v}(\mathbf{x})=\sum_{\mathbf{k} \in \mathbb{Z}^{2}} v_{\mathbf{k}} \mathbf{e}_{\mathbf{k}}
$$


where $\mathbf{e}_{\mathbf{k}}$ is the divergence-free basis function

$$
\mathbf{e}_{\mathbf{k}}(\mathbf{x})=\frac{\mathbf{k}^{\perp}}{\|\mathbf{k}\|} e^{2 \pi i \mathbf{k} \cdot \mathbf{x}} .
$$

To apply Bayes' Theorem to function spaces, we must define probability measures on those spaces. A natural approach, described in detail in Section 2 of [24], is to construct an infinite-dimensional probability measure via probability measures on individual components of a basis for the function space. Thus, probability measures on the space of divergencefree vector fields can be defined by letting $v_{\mathbf{k}}$ be random fields, as long as $v_{\mathbf{k}}$ exhibit the convergence as $\|\mathbf{k}\| \rightarrow \infty$ given in (3.3). An example probability measure on the space of divergence-free vector fields, defined using this approach, is described in Section 8.1.

\subsection{Mathematical Setting}

In this section, we define weak solutions $\theta$ of the advection-diffusion problem and show that for $\mathbf{v} \in H$ and assuming sufficient smoothness of the initial condition $\theta_{0}$, observations of such solutions exhibit some basic continuity properties.

Definition 3.3 (Weak Solution, $\theta$ ). Let $\mathbf{v} \in H$ and $\theta_{0} \in H^{3+\epsilon}$ as defined in Definition 3.2 and Definition 3.1, respectively. Then we say that $\theta \in L^{2}\left([0, T] ; H^{1}\left(\mathbb{T}^{2}\right)\right)$ is a weak solution of (1.1) provided that $\theta(0)=\theta_{0}$ and

$$
\left\langle\frac{\partial}{\partial t} \theta, \phi\right\rangle_{H^{-1}\left(\mathbb{T}^{2}\right) \times H^{1}\left(\mathbb{T}^{2}\right)}+\langle\mathbf{v} \cdot \nabla \theta, \phi\rangle_{L^{2}\left(\mathbb{T}^{2}\right)}+\kappa\langle\nabla \theta, \nabla \phi\rangle_{L^{2}\left(\mathbb{T}^{2}\right)}=0
$$

for all $\phi \in H^{1}\left(\mathbb{T}^{2}\right)$ and almost all time $t \in[0, T]$.

Remark 3.4 (Continuity of $\mathbf{v}, \theta_{0}$ ). Note that by Sobolev embedding (see [74], Theorem 
A.2), $\mathbf{v} \in C^{1}\left(\mathbb{T}^{2}\right)$ and $\theta_{0} \in C^{2}\left(\mathbb{T}^{2}\right)$.

We also note that under the above assumptions on $\mathbf{v}$ and $\theta_{0}$, the solution $\theta$ is continuous.

Remark 3.5 (Continuity of $\theta$ ). Let $\mathbf{v} \in H$ and $\theta_{0} \in H^{3+\epsilon}$. Then

$$
\theta \in L^{\infty}\left(0, T ; H^{2}\left(\mathbb{T}^{2}\right)\right)
$$

(see [31], Section 7.1, Theorem 5). Then by [31], Section 5.9, Theorem 4,

$$
\theta \in C\left(0, T ; H^{1}\left(\mathbb{T}^{2}\right)\right) \subset C\left(0, T ; C\left(\mathbb{T}^{2}\right)\right)
$$

since $H^{1}\left(\mathbb{T}^{2}\right) \subset C\left(\mathbb{T}^{2}\right)$ (see [74], Theorem A.1).

We now introduce some notation.

Definition 3.6 (Solution Operator $\mathcal{S}$, Observation Operator $\mathcal{O}$ ). The forward map $\mathcal{G}$ can be interpreted as the composition of two operators:

$$
\mathcal{G}(\mathbf{v})=\mathcal{O} \circ \mathcal{S}(\mathbf{v})
$$

where:

1. The solution operator $\mathcal{S}: H \rightarrow L^{2}\left([0, T] ; H^{1}\left(\mathbb{T}^{2}\right)\right)$ maps a given $\mathbf{v}$ to its corresponding $\theta$ (the solution of $(1.1))$.

2. The observation operator $\mathcal{O}: L^{2}\left([0, T] ; H^{1}\left(\mathbb{T}^{2}\right)\right) \mapsto Y$ measures some quantities (e.g. point measurements, spectral data, tracers) from $\theta$. 
This paper will focus on point observations $\mathcal{G}_{j}(\mathbf{v})=\theta\left(t_{j}, \mathbf{x}_{j}, \mathbf{v}\right), j=1, \ldots, N$ as the most obvious practical implementation for the advection-diffusion problem; note that by Remark 3.5 such observations are well-defined. We now show that, under the above assumptions on $\mathbf{v}$ and $\theta_{0}$, point observations also vary continuously with $\mathbf{v}$. To do so, we will use a particle interpretation of the advection-diffusion equation (1.1) and Kolmogorov's Backward Equation (see, for example, Section 8.1 of [68]), which describes the relationship between the concentration of a solute $\theta(t, \mathbf{x})$ and the motion of individual solution particles, denoted by $\mathbf{X}_{t}$. The equation uses the Brownian motion denoted by $\mathbf{W}_{t}$.

Theorem 3.7 (Kolmogorov's Backward Equation). Suppose that for all $t>0, \mathbf{x} \in \mathbb{T}^{2}, \theta$ satisfies (1.1) with $\theta_{0} \in C^{2}\left(\mathbb{T}^{2}\right), \mathbf{v} \in C^{1}\left(\mathbb{T}^{2}\right)$. Then $\theta(t, \mathbf{x})$ is given by

$$
\theta(t, \mathbf{x})=\mathbb{E}\left[\theta_{0}\left(\mathbf{X}_{t}\right)\right]
$$

where $\mathbf{X}_{t}$ is the Itô diffusion

$$
d \mathbf{X}_{t}=-\mathbf{v}\left(\mathbf{X}_{t}\right) d t+\sqrt{2 \kappa} d \mathbf{W}_{t}, \quad \mathbf{X}_{0}=\mathbf{x}
$$

Lemma 3.8 (Continuity of Solution Map into $L^{\infty}$ ). Let $\theta_{0} \in H^{3+\epsilon}$ and $\mathbf{v} \in H$, as defined in Definition 3.1 and Definition 3.2, respectively. Then the solution map $\mathcal{S}: H \rightarrow$ $L^{\infty}\left([0, T] \times \mathbb{T}^{2}\right)$ given by $\mathcal{S}: \mathbf{v} \mapsto \theta(\mathbf{v})$ is continuous.

Proof. Let $\mathbf{X}_{t}^{\mathbf{v}}, \mathbf{X}_{t}^{\tilde{\mathbf{v}}}$ respectively satisfy

$$
\begin{array}{ll}
d \mathbf{X}_{t}^{\mathbf{v}}=-\mathbf{v}\left(\mathbf{X}_{t}^{\mathbf{v}}\right) d t+\sigma\left(\mathbf{X}_{t}^{\mathbf{v}}\right) d \mathbf{W}_{t}, & \mathbf{X}_{0}^{\mathbf{v}}=\mathbf{x} \\
d \mathbf{X}_{t}^{\tilde{\mathbf{v}}}=-\tilde{\mathbf{v}}\left(\mathbf{X}_{t}^{\tilde{\mathbf{v}}}\right) d t+\sigma\left(\mathbf{X}_{t}^{\tilde{\mathbf{v}}}\right) d \mathbf{W}_{t}, & \mathbf{X}_{0}^{\tilde{\mathbf{v}}}=\mathbf{x}
\end{array}
$$


Denote $\mathbf{Y}_{t}=\mathbf{X}_{t}^{\mathbf{v}}-\mathbf{X}_{t}^{\tilde{\mathbf{v}}}$. Then

$$
\frac{d}{d t}\left|\mathbf{Y}_{t}\right|=\left|\tilde{\mathbf{v}}\left(\mathbf{X}_{t}^{\tilde{\mathbf{v}}}\right)-\mathbf{v}\left(\mathbf{X}_{t}^{\mathbf{v}}\right)\right|
$$

so that

$$
\begin{aligned}
\frac{1}{2} \frac{d}{d t}\left\|\mathbf{Y}_{t}\right\|^{2} & =\left\langle\mathbf{Y}_{t}, \tilde{\mathbf{v}}\left(\mathbf{X}_{t}^{\tilde{v}}\right)-\mathbf{v}\left(\mathbf{X}_{t}^{\mathbf{v}}\right)\right\rangle \\
& =\left\langle\mathbf{Y}_{t}, \tilde{\mathbf{v}}\left(\mathbf{X}_{t}^{\tilde{\mathbf{v}}}\right)-\tilde{\mathbf{v}}\left(\mathbf{X}_{t}^{\mathbf{v}}\right)\right\rangle+\left\langle\mathbf{Y}_{t}, \tilde{\mathbf{v}}\left(\mathbf{X}_{t}^{\mathbf{v}}\right)-\mathbf{v}\left(\mathbf{X}_{t}^{\mathbf{v}}\right)\right\rangle \\
& \leq\left\|\mathbf{Y}_{t}\right\|\left\|\tilde{\mathbf{v}}\left(\mathbf{X}_{t}^{\tilde{\mathbf{v}}}\right)-\tilde{\mathbf{v}}\left(\mathbf{X}_{t}^{\mathbf{v}}\right)\right\|+\left\|\mathbf{Y}_{t}\right\|\left\|\tilde{\mathbf{v}}\left(\mathbf{X}_{t}^{\mathbf{v}}\right)-\mathbf{v}\left(\mathbf{X}_{t}^{\mathbf{v}}\right)\right\| \\
& \leq\left\|\mathbf{Y}_{t}\right\|\left\|\tilde{\mathbf{v}}\left(\mathbf{X}_{t}^{\tilde{\mathbf{v}}}\right)-\tilde{\mathbf{v}}\left(\mathbf{X}_{t}^{\mathbf{v}}\right)\right\|+\left\|\mathbf{Y}_{t}\right\|\|\tilde{\mathbf{v}}-\mathbf{v}\|_{L^{\infty}} .
\end{aligned}
$$

By the Mean Value Theorem, there exists a $\mathbf{z}$ such that

$$
\tilde{\mathbf{v}}\left(\mathbf{X}_{t}^{\tilde{\mathbf{v}}}\right)-\tilde{\mathbf{v}}\left(\mathbf{X}_{t}^{\mathbf{v}}\right)=\nabla \mathbf{v}(\mathbf{z})\left(\mathbf{X}_{t}^{\tilde{\mathbf{v}}}-\mathbf{X}_{t}^{\mathbf{v}}\right)=\nabla \mathbf{v}(\mathbf{z}) \mathbf{Y}_{t}
$$

Therefore

$$
\frac{d}{d t}\left\|\mathbf{Y}_{t}\right\| \leq\left\|\nabla \mathbf{v}(\mathbf{z})\left(\mathbf{X}_{t}^{\tilde{\mathbf{v}}}-\mathbf{X}_{t}^{\mathbf{v}}\right)\right\|+\|\tilde{\mathbf{v}}-\mathbf{v}\|_{L^{\infty}} \leq\|\nabla \mathbf{v}\|_{L^{\infty}}\left\|\mathbf{Y}_{t}\right\|+\|\tilde{\mathbf{v}}-\mathbf{v}\|_{L^{\infty}}
$$

Integrating in time and using Gronwall's inequality gives

$$
\left\|\mathbf{X}_{T}^{\mathbf{v}}-\mathbf{X}_{T}^{\tilde{\mathbf{v}}}\right\| \leq T e^{T\|\nabla \mathbf{v}\|_{L^{\infty}}}\|\tilde{\mathbf{v}}-\mathbf{v}\|_{L^{\infty}}
$$

Then, applying Kolmogorov's Backward Equation and again using the Mean Value Theorem, 
there exists a $\tilde{\mathbf{z}}$ such that

$$
\begin{aligned}
|\theta(t, \mathbf{x}, \tilde{\mathbf{v}})-\theta(t, \mathbf{x}, \mathbf{v})| & =\left|\mathbb{E} \theta_{0}\left(\mathbf{X}_{t}^{\tilde{\mathbf{v}}}\right)-\theta_{0}\left(\mathbf{X}_{t}^{\mathbf{v}}\right)\right| \\
& =\left|\mathbb{E} \nabla \theta_{0}(\tilde{\mathbf{z}})\left(\mathbf{X}_{t}^{\tilde{\mathbf{v}}}-\mathbf{X}_{t}^{\mathbf{v}}\right)\right| \\
& \leq\left\|\nabla \theta_{0}\right\|_{L^{\infty}} T e^{T\|\nabla \mathbf{v}\|_{L^{\infty}}}\|\tilde{\mathbf{v}}-\mathbf{v}\|_{L^{\infty}} . \\
& \leq C_{a} C_{c}\left\|\theta_{0}\right\|_{H^{3+\epsilon}} T e^{C_{b} T\|\mathbf{v}\|_{H}}\|\tilde{\mathbf{v}}-\mathbf{v}\|_{H}
\end{aligned}
$$

where

$$
\left\|\nabla \theta_{0}\right\|_{L^{\infty}} \leq C_{a}\left\|\theta_{0}\right\|_{H^{3+\epsilon}}, \quad\|\nabla \mathbf{v}\|_{L^{\infty}} \leq C_{b}\|\mathbf{v}\|_{H}, \quad\|\tilde{\mathbf{v}}-\mathbf{v}\|_{L^{\infty}} \leq C_{c}\|\tilde{\mathbf{v}}-\mathbf{v}\|_{H}
$$

for some constants $C_{a}, C_{b}, C_{c}$ (see [74], Corollary A.2).

Corollary 3.9 (Continuity of Solution Map into $L^{2}$ ). Let $\theta_{0} \in H^{3+\epsilon}$ and $\mathbf{v} \in H$, as defined in Definition 3.1 and Definition 3.2, respectively. Then the solution map $\mathcal{S}: H \rightarrow$ $L^{2}\left([0, T] \times \mathbb{T}^{2}\right)$ given by $\mathcal{S}: \mathbf{v} \mapsto \theta(\mathbf{v})$ is continuous.

Corollary 3.10 (Continuity of Point Observations). Let $\theta_{0} \in H^{3+\epsilon}$ and $\mathbf{v} \in H$, as defined in Definition 3.1 and Definition 3.2, respectively. Then the map $\mathcal{G}_{j}: H \rightarrow \mathbb{R}$ given by $\mathcal{G}_{j}: \mathbf{v} \mapsto \theta\left(t_{j}, \mathbf{x}_{j}, \mathbf{v}\right)$ is continuous

\subsection{Ill-posedness of the Inverse Problem}

In this section, we will briefly describe why the inverse problem is ill-posed, i.e., in general, we cannot determine the background flow that generated the data, represented by $\mathbf{v}^{\star}$ here and in the remainder of the dissertation.

Definition 3.11 (True background flow, $\mathbf{v}^{\star}$ ). We denote the "true" background flow by $\mathbf{v}^{\star}$, 
so that

$$
\mathcal{Y}=\mathcal{G}\left(\mathbf{v}^{\star}\right)+\eta
$$

(though $\mathbf{v}^{\star}$ is not necessarily the only $\mathbf{v}$ that satisfies this relationship).

In an ideal setting, where we could take infinite, noise-free observations that uniquely define the background flow, we would simply invert the forward model $\mathcal{G}$ to obtain $\mathbf{v}^{\star}$. (The behavior of Bayesian inference for a large number of observations is investigated further in Chapter 4.) However, in practice the desired inference is ill-posed in a number of ways, as we will describe here.

First, note that the observation operator $\mathcal{O}$ maps an infinite-dimensional space to a finitedimensional one. As a result, $\mathcal{G}$ will not in general be invertible. This points to a key consideration in the inversion:

Consideration 3.12. Even in the absence of noise $(\eta=0)$, there may be many background flows $\mathbf{v}$ that produce data $\mathcal{Y}$.

Section 8.3 provides one such example, where symmetries in the problem make it impossible for observations to discern between two background flows. Moreover, in some cases, the map $\mathbf{v} \mapsto \theta$ will not be invertible even for infinite observations; two such examples are provided in Section 4.1.

Secondly, observation noise $\eta$ will in general be non-zero. This leads to another consideration:

Consideration 3.13. Given observation noise $(\eta \neq 0)$, there may be no background flows $\mathbf{v}$ that produce data $\mathcal{Y}$. That is, there may exist $\mathbf{v}^{\star}, \eta$ such that $\mathcal{Y}=\mathcal{G}\left(\mathbf{v}^{\star}\right)+\eta \notin \operatorname{Ran}(\mathcal{G})$.

For an example of such a case, consider $\mathcal{O}$ consisting of point observations $\theta\left(t_{j}, \mathbf{x}_{j}\right)$. We may find that, due to observational noise, we have a point measurement that exceeds the 
maximum value (or is less than the minimum value) of $\theta_{0}$. However, because $\nabla \cdot \mathbf{v}=0,(1.1)$ is a parabolic PDE that is subject to a maximum principle - in the absence of sources or sinks in the background flow, pollutants do not spontaneously collect in a given location so that $\|\theta(t)\|_{L^{\infty}\left(\mathbb{T}^{2}\right)} \leq\left\|\theta_{0}\right\|_{L^{\infty}\left(\mathbb{T}^{2}\right)}$ for all $t>0$. Thus there would be no such $\mathbf{v}$ such that $\mathcal{G}(\mathbf{v})=\mathcal{Y}$. This is illustrated in Figure 3.1.

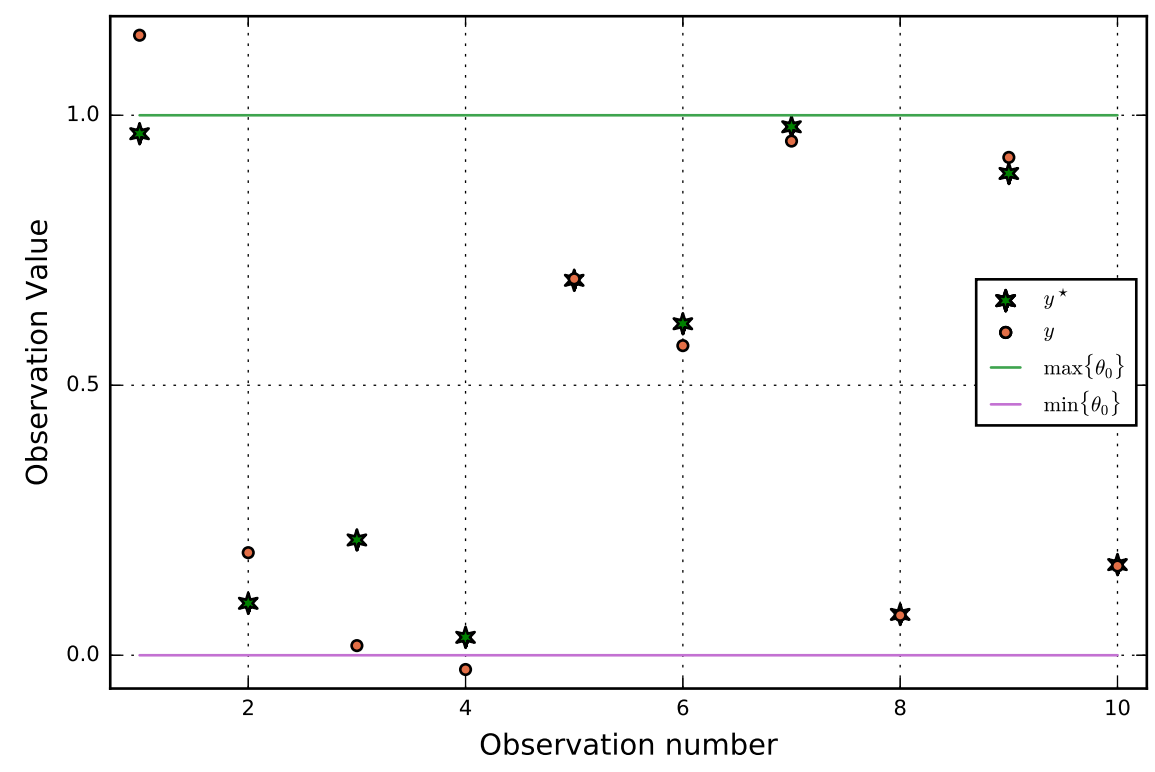

Figure 3.1: Illustration of Consideration 3.13: The addition of noise to perfect measurements $\mathcal{Y}^{\star}$ drives some of the observations $\mathcal{Y}$ outside the range of $\theta_{0}$, violating the maximum principle.

These two considerations are typical of ill-posed inverse problems (see, e.g. Section 2 of [46]). The next section describes the Bayesian approach, and how it helps regularize the problem.

\subsection{Bayesian Inference}

As described in Section 3.3, the inverse problem is, in general, ill-posed: There may be many $\mathbf{v}$ such that $\mathcal{G}(\mathbf{v})=\mathcal{Y}$, or none at all. In this section, we describe the Bayesian approach to inverse problems, and how it addresses the considerations laid out in the previous section. 
We refer the reader to [46] as a reference on statistical inverse problems and to [24, 80] for a thorough discussion of such problems in infinite dimensions.

In practice, when inverse problems are ill-posed, the data are interpreted in context - the practitioner will need to understand (i) what is and is not known about the solution in the absence of any measurements, and also (ii) what the data does and not imply about the solution. Since noisy measurements do not uniquely specify a solution, the "right" answer to the inverse problem incorporates both (i) and (ii). This is the process that Bayesian inference quantifies. For (i), we introduce a measure $\mu_{0}$, called the prior, on $H$ that represents our best information of the value of $\mathbf{v}$ before data are incorporated into the model. This may be based on past/historical data for the system or using "expert" opinion. For (ii), we define the answer to the inverse problem as not a single $\mathbf{v}$, but rather a posterior measure $\mu_{\mathcal{Y}}$ (the measure associated with the random variable $\mathbf{v} \mid \mathcal{Y}$ ) on $H$ that represents the probability that the background flow $\mathbf{v}$ lies in a given subset of $H$, incorporating both the prior (expert opinion) and the measured data. This requires a probabilistic model for the noise $\eta$.

If the data are not very informative - that is, if they are sparse or very noisy - the expert judgement may not be significantly influenced by the observations and the posterior may be very similar to the prior. If the data are very informative - high-dimensional and measured with high-precision equipment - then the posterior may be substantially different from the prior. In fact, in the informative case, the problem approaches a traditional inverse problem, up to the constraints posed by trying to infer high-dimensional $\mathbf{v}$ from lower-dimensional $\mathcal{Y}$. This large observation limit is investigated in more detail in Chapter 4.

If a single answer is desired at the end of a Bayesian inference, any of a number of methods can be used to extract one, a few of the most prominent being:

- The mean value according to the posterior measure. 
- Maximum a posteriori (MAP) estimator [28]: The value that maximizes probability under the posterior measure. In the advection-diffusion problem, this would be the $\mathbf{v}$ that is most likely given both the prior and the measured data.

- Maximum likelihood estimator (MLE) [75]: The value that maximizes the likelihood. In the advection-diffusion problem, this is the $\mathbf{v}$ that best matches the data.

\subsubsection{Regularization}

In this section, we describe qualitatively how the Bayesian approach regularizes the inverse problem - that is, addresses the ill-posedness identified in Consideration 3.12 and Consideration 3.13.

The first is straightforward: Consideration 3.12 notes that multiple solutions $\mathbf{v}$ may match the data $\mathcal{Y}$ equally well. In the Bayesian framework, the prior provides a means of determining the relative probability of such solutions. Roughly, if the data does not distinguish between two values, then their relative probability given the data will be unchanged from before the data were collected. If both the prior and the data match two solutions equally well, then those solutions will be equally likely according to the posterior; see Section 8.3 for one such example. A more detailed discussion of the affect of the prior on Bayesian inference is provided in Section 2 of [80].

Consideration 3.13 is addressed by taking a probabilistic interpretation of the noise $\eta$. Let $\mathbb{Q}_{0}$ denote the measure associated with the probability distribution of the noise $\eta$, i.e. $\eta \sim \mathbb{Q}_{0}$ and assume $\eta$ is independent of $\mathcal{Y}$. That is, $\mathbb{Q}_{0}$ describes the accuracy of the measurements - a "narrow" $\mathbb{Q}_{0}$ means smaller noise and more accurate measurements. Then we have $\mathcal{Y}-\mathcal{G}(\mathbf{v}) \sim \mathbb{Q}_{0}$ - that is, we can evaluate the likelihood that the background flow takes a given value $\mathbf{v}$ by evaluating the probability of $\mathcal{Y}-\mathcal{G}(\mathbf{v})$ according to $\mathbb{Q}_{0}$. Then instead of seeking 
$\mathbf{v}$ such that $\mathcal{G}(\mathbf{v})=\mathcal{Y}$, we weight $\mathbf{v}$ in our posterior by the probability of $\mathcal{Y}-\mathcal{G}(\mathbf{v})$ according to $\mathbb{Q}_{0}$. Thus, we need not have $\mathcal{Y}=\mathcal{G}(\mathbf{v})+\eta \in \operatorname{Ran}(\mathcal{G})$, addressing Consideration 3.13.

An equivalent approach is to shift $\mathbb{Q}_{0}$ by $\mathcal{G}(\mathbf{v})$, which we will define next.

Definition 3.14 (Shifted Noise Measure $\mathbb{Q}_{\mathbf{v}}$ ). Given background flow $\mathbf{v} \in H$ and observation noise $\eta \sim \mathbb{Q}_{0}$, define a shifted noise measure $\mathbb{Q}_{\mathbf{v}}$ that satisfies

$$
\mathcal{G}(\mathbf{v})+\eta \sim \mathbb{Q}_{\mathbf{v}}
$$

We can then evaluate $\mathbb{Q}_{\mathbf{v}}$ at $\mathcal{Y}$ to yield the distribution of $\mathcal{Y} \mid \mathbf{v}$. This approach will be used in the derivation of Bayes' Theorem in the next section.

\subsubsection{Bayes' Theorem}

In this section, we lay out an infinite-dimensional version of the classical Bayes' Theorem, following closely the derivation in [24]. We begin by making the following assumption:

Assumption 3.15. The shifted noise measure $\mathbb{Q}_{\mathbf{v}}$ (see Definition 3.14) is absolutely continuous with respect to the unshifted noise measure $\mathbb{Q}_{0}$, for $\mathbf{v} \mu_{0}-$ a.s..

This implies that the Radon-Nikodym derivative $\frac{d \mathbb{Q}_{v}}{d \mathbb{Q}_{0}}$ exists. We note that this assumption holds when $\mathbb{Q}_{0}$ is any measure, such as a (non-degenerate) Gaussian, that has the whole space $Y$ as its support. Then we define:

Definition 3.16 (Potential, $\Phi)$. When Assumption 3.15 holds, the potential or negative 
$\log$-likelihood $\Phi:(H \times Y) \rightarrow \mathbb{R}$ is defined as

$$
\frac{d \mathbb{Q}_{\mathbf{v}}}{d \mathbb{Q}_{0}}(\mathcal{Y})=\exp (-\Phi(\mathbf{v} ; \mathcal{Y}))
$$

$\Phi$ is defined with an exponential here because of an analogy with potential energy that will become more clear in later sections; see, e.g., the discussions of the MALA or HMC methods in, respectively, Section 5.4 and Section 5.5. Also, in practice logarithms of probabilities are often computed to avoid numerical errors resulting from working with values of the probability that are very small.

Then we define the following product measures:

$$
\nu_{\mathbf{v}}(d \mathbf{v}, d \mathcal{Y})=\mu_{0}(d \mathbf{v}) \mathbb{Q}_{\mathbf{v}}(d \mathcal{Y}), \quad \nu_{0}(d \mathbf{v}, d \mathcal{Y})=\mu_{0}(d \mathbf{v}) \mathbb{Q}_{0}(d \mathcal{Y})
$$

We further assume:

Assumption 3.17. $\Phi$ is $\nu_{0}$-measurable, and

$$
Z=\int \exp (-\Phi(\mathbf{v} ; \mathcal{Y})) \mu_{0}(d \mathbf{v})>0 \quad \text { for } \mathcal{Y} \mathbb{Q}_{0}-\text { a.s. }
$$

Note that $\nu_{\mathbf{v}}$ describes the distribution of the random variable $(\mathbf{v}, \mathcal{Y})$. By Assumption 3.15, $\nu_{\mathbf{v}}$ is absolutely continuous with respect to $\nu_{0}$ and, under Assumption 3.17, their RadonNikodym derivative is given by $\frac{d \mathbb{Q}_{v}}{d \mathbb{Q}_{0}}=\exp (-\Phi)$.

Theorem 3.18 (Bayes' Theorem, [24]). Let $\mathbb{Q}_{\mathbf{v}}$ and $\Phi$ be defined as in Definition 3.14 and Definition 3.16, respectively, and satisfy Assumption 3.15 and Assumption 3.1\%. 
Then the measure $\mu_{\mathcal{Y}}$ associated with the random variable $\mathbf{v} \mid \mathcal{Y}$ exists under $\nu_{\mathbf{v}}$, and $\mu_{\mathcal{Y}}$ is absolutely continuous with respect to $\mu_{0} \nu_{\mathbf{v}}$ a.s., with Radon-Nikodym derivative

$$
\frac{d \mu \mathcal{Y}}{d \mu_{0}}(\mathbf{v})=\frac{1}{Z} \exp (-\Phi(\mathbf{v} ; \mathcal{Y}))
$$

where the normalization factor $Z$ is as defined in (3.24).

Example 3.19 (Gaussian Noise). If we assume that the observation noise is a centered Gaussian, i.e. $\mathbb{Q}_{0}=N\left(0, \mathcal{C}_{\eta}\right)$ then we have $\mathbb{Q}_{\mathbf{v}}=N\left(\mathcal{G}(\mathbf{v}), \mathcal{C}_{\eta}\right)$, so

$$
\Phi(\mathbf{v} ; \mathcal{Y})=\frac{1}{2}\left\|\mathcal{C}_{\eta}^{-1 / 2}(\mathcal{Y}-\mathcal{G}(\mathbf{v}))\right\|^{2}-\frac{1}{2}\left\|\mathcal{C}_{\eta}^{-1 / 2} \mathcal{Y}\right\|^{2}
$$

Moreover, because it is finite $\mathbb{Q}_{0}$-a.s., the second term above can be absorbed into the normalization factor $Z$, yielding simply

$$
\Phi(\mathbf{v} ; \mathcal{Y})=\frac{1}{2}\left\|\mathcal{C}_{\eta}^{-1 / 2}(\mathcal{Y}-\mathcal{G}(\mathbf{v}))\right\|^{2} .
$$

Example 3.20 (Gaussian Prior and Noise). For Gaussian prior $\mu_{0}=N\left(0, \mathcal{C}_{0}\right)$ and Gaussian observation noise $\eta \sim N\left(0, \mathcal{C}_{\eta}\right)$ the point of maximum probability under $\mu_{\mathcal{Y}}$ can be found by solving

$$
\underset{\mathbf{v}}{\arg \min }\left[\frac{1}{2}\left\|\mathcal{C}_{\eta}^{-1 / 2}(\mathcal{Y}-\mathcal{G}(\mathbf{v}))\right\|^{2}+\frac{1}{2}\left\|\mathcal{C}_{0}^{-1 / 2} \mathbf{v}\right\|^{2}\right]
$$

Thus, finding the point of maximum probability under the posterior (maximum a posteriori, or MAP, point) is equivalent to solving the Tikhonov regularization problem (3.28).[24] 


\section{Chapter 4}

\section{Consistency of the Bayesian Posterior}

In this section, we show conditions under which the Bayesian posterior converges weakly to a Dirac measure centered on the true vector field $\mathbf{v}^{\star}$ (see Definition 3.11) as the number of observations goes to infinity. That is, under these conditions, the observation error is averaged away, the effect of the prior on the posterior is eliminated, and the true vector field is uniquely identified by the data. The key result is shown in Theorem 4.22.

We begin by defining an additional space that will be used in the proof; in particular, this space will be used to restrict $\mathbf{v}$ to a compact set to ensure both continuity of the inverse map $\theta \mapsto \mathbf{v}$ and convergence of the log-likelihood that is uniform across $\mathbf{v}$. Both of these properties will be required to show weak convergence of the posterior to a Dirac measure.

Definition $4.1\left(V, B_{V}^{r}\right)$. Define the space

$$
V=\left\{\mathbf{v} \in H^{3+\epsilon}\left(\mathbb{T}^{2}\right)^{2}: \nabla \cdot \mathbf{v}=0\right\}
$$

and denote the ball about $\mathbf{v}_{0} \in V$ of radius $r>0$ by

$$
B_{V}^{r}\left(\mathbf{v}_{0}\right)=\left\{\mathbf{v} \in V:\left\|\mathbf{v}-\mathbf{v}_{0}\right\|_{V} \leq r\right\} .
$$

Lemma 4.2. Let $r>0$ and $\mathbf{v}_{0} \in V$. Then $B_{V}^{r}\left(\mathbf{v}_{0}\right)$ is compact in $H$ (see Definition 3.2). 
Proof. This is an application the Rellich-Kondrachov Theorem; see, e.g., Corollary A.5 of $[74]$.

In what follows, we will also assume that the true background flow $\mathbf{v}^{\star}$ has additional regularity.

Assumption 4.3 (Regularity of $\mathbf{v}^{\star}$ ). The true background flow $\mathbf{v}^{\star}$ (Definition 3.11) satisfies $\mathbf{v}^{\star} \in V$.

\subsection{Continuity of Inverse Map}

In this section, we lay out conditions under which the inverse solution map $\theta \mapsto \mathbf{v}$ is continuous. First, we provide two examples to illustrate potential issues with the inverse problem that result in the assumptions that we make in the proof.

Since the background flow $\mathbf{v}$ enters (1.1) through the $\mathbf{v} \cdot \nabla \theta$ term, the inverse problem can be ill-posed when this term is small. The most basic such case is where $\mathbf{v} \cdot \nabla \theta$ is zero everywhere, in which case the vector field does not have any effect on $\theta$. Figure 4.1 and Figure 4.2 show two such examples, in which any multiple of $\mathbf{v}^{\star}$ will produce the same scalar behavior. In these cases, the observations have no way to discriminate between a range of background flows, and so the posterior cannot identify $\mathbf{v}^{\star}$ for any number of observations (except for very particular priors).

With these examples in mind, in Assumption 4.4 and Definition 4.5 we construct data that consists of observations with two initial conditions $\theta_{0}^{(1)}, \theta_{0}^{(2)}$ such that $\mathbf{v} \cdot \nabla \theta_{0}^{(i)} \neq 0$ for $i=1$ and/or 2 wherever $\mathbf{v} \neq 0$ (up to a set of measure zero). The multiple initial conditions can be interpreted as an experiment where a contaminant is injected into a system multiple times to observe its behavior. 

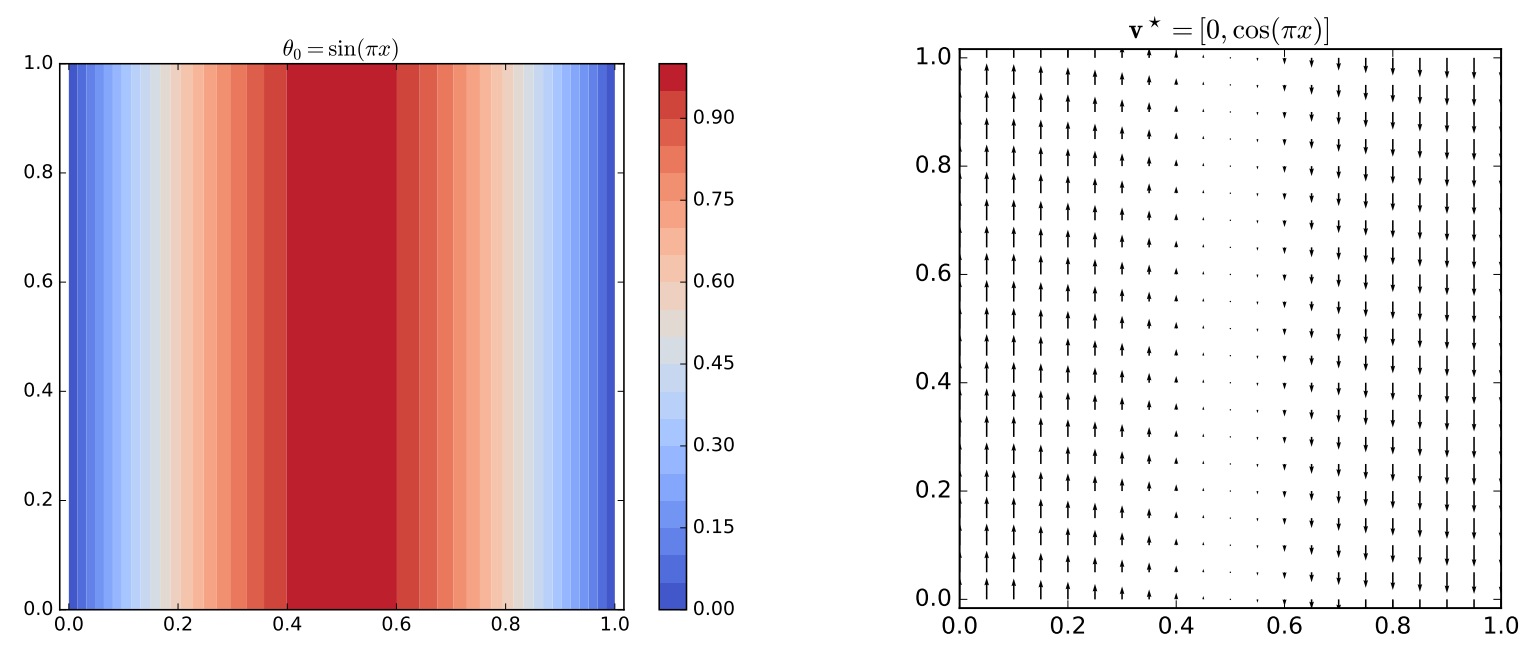

Figure 4.1: Ill-posedness Example \#1: Any $\mathbf{v}=[0, f(x)]$ produces the same $\theta$.
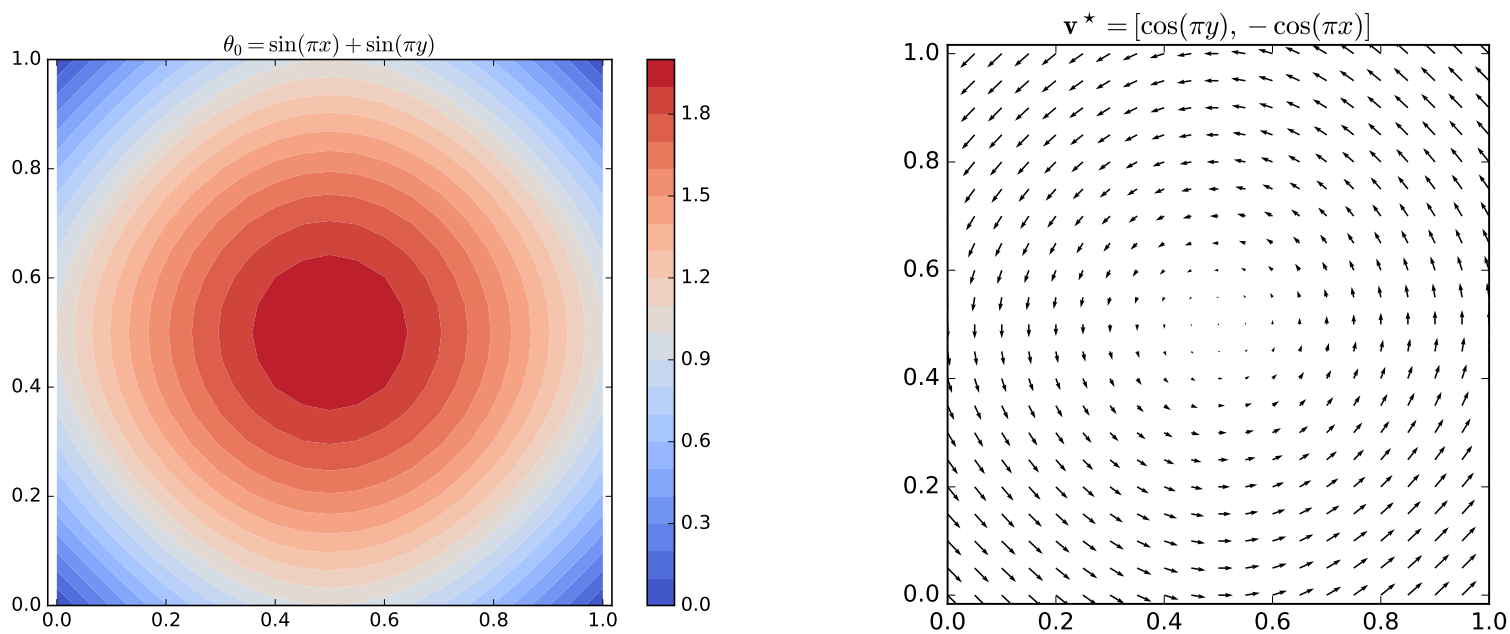

Figure 4.2: Ill-posedness Example \#2: Any multiple of $\mathbf{v}^{\star}$ produces the same $\theta$. 
Assumption 4.4 (Two initial conditions). Let $\theta^{(1)}, \theta^{(2)}$ be the solutions to the advectiondiffusion equation (1.1) with the initial conditions $\theta_{0}^{(1)}(\mathbf{x})$ and $\theta_{0}^{(2)}(\mathbf{x})$ such that for almost all $\mathbf{x} \in \mathbb{T}^{2}$,

$$
\nabla \theta_{0}^{(1)}(\mathbf{x}) \times \nabla \theta_{0}^{(2)}(\mathbf{x}) \neq 0
$$

Definition 4.5 (Point observations for multiple initial conditions, $\mathcal{G}_{j}$ ). Let $\theta^{(1)}, \theta^{(2)}$ be as defined in Assumption 4.4. Let $\left\{\left(t_{j}, \mathbf{x}_{j}\right)\right\}_{j=1}^{N}$ be a sequence of observation points. Define the parameter-to-observable (forward) map $\mathcal{G}_{j}$ for point observations from multiple initial conditions by

$$
\begin{array}{r}
\mathcal{G}_{2 j-1}(\mathbf{v})=\theta^{(1)}\left(t_{j}, \mathbf{x}_{j}, \mathbf{v}\right) \\
\mathcal{G}_{2 j}(\mathbf{v})=\theta^{(2)}\left(t_{j}, \mathbf{x}_{j}, \mathbf{v}\right)
\end{array}
$$

for $j=1, \ldots, N$.

We now define the solution map associated with the solution of (1.1) for the multiple initial conditions given in Assumption 4.4.

Definition 4.6 (Paired solution map $\tilde{\mathcal{S}})$. Let $\tilde{\mathcal{S}}: H \rightarrow L^{2}\left([0, T] \times \mathbb{T}^{2}\right)^{2}$ be the map $\tilde{\mathcal{S}}: \mathbf{v} \mapsto$ $\left(\theta^{(1)}(\mathbf{v}), \theta^{(2)}(\mathbf{v})\right)$, where $\theta^{(i)}$ for $i=1,2$ is given by the solution to the advection-diffusion equation (1.1) with initial condition $\theta_{0}^{(i)}$ :

$$
\frac{\partial}{\partial t} \theta^{(i)}=-\mathbf{v} \cdot \nabla \theta^{(i)}+\kappa \Delta \theta^{(i)} \quad, \quad \theta(0)=\theta_{0}^{(i)}
$$

We now show that the the paired solution map $\tilde{\mathcal{S}}$ is continuous (Corollary 4.7) and 1-to-1 (Lemma 4.8), and therefore the inverse of $\tilde{\mathcal{S}}$ is continuous (Corollary 4.10). 
Corollary $4.7(\tilde{\mathcal{S}}$ continuous $)$. The paired solution map $\tilde{\mathcal{S}}: H \rightarrow L^{2}\left([0, T] \times \mathbb{T}^{2}\right)^{2}($ see Definition 4.6) is continuous.

Proof. The solution map $\mathcal{S}: H \rightarrow L^{2}\left([0, T] \times \mathbb{T}^{2}\right)$ given by $\mathbf{v} \mapsto \theta(\mathbf{v})$ is continuous by Corollary 3.9. So the map $\tilde{\mathcal{S}}: \mathbf{v} \mapsto\left(\theta^{(1)}(\mathbf{v}), \theta^{(2)}(\mathbf{v})\right)$ is also continuous.

Lemma 4.8 ( $\tilde{\mathcal{S}}$ injective). Let $\tilde{\mathcal{S}}$ be the paired solution map given in Definition 4.6 with initial conditions satisfying Assumption 4.4. Suppose that $\mathbf{v}, \tilde{\mathbf{v}} \in H$ such that

$$
\|\tilde{\mathcal{S}}(\mathbf{v})-\tilde{\mathcal{S}}(\tilde{\mathbf{v}})\|_{L^{2}\left([0, T] \times \mathbb{T}^{2}\right)^{2}}=0
$$

Then $\|\mathbf{v}-\tilde{\mathbf{v}}\|_{H}=0$.

Proof. Let $\mathbf{v}, \tilde{\mathbf{v}} \in H$ satisfy (4.6), i.e.,

$$
\left\|\theta^{(i)}(\cdot, \mathbf{v})-\theta^{(i)}(\cdot, \tilde{\mathbf{v}})\right\|_{L^{2}\left([0, T] \times \mathbb{T}^{2}\right)}=0, \quad i=1,2 .
$$

Then $\theta^{(i)}(t, \mathbf{x}, \mathbf{v})=\theta^{(i)}(t, \mathbf{x}, \tilde{\mathbf{v}})$ for almost all $t, \mathbf{x}$ and $i=1,2$. However, since both solutions are continuous (see Remark 3.5), this implies that $\theta^{(i)}(t, \mathbf{x}, \mathbf{v})=\theta^{(i)}(t, \mathbf{x}, \tilde{\mathbf{v}})$ for all $t, \mathbf{x}$ and $i=1,2$. Denote $\theta^{(i)}(t, \mathbf{x}):=\theta^{(i)}(t, \mathbf{x}, \mathbf{v})=\theta^{(i)}(t, \mathbf{x}, \tilde{\mathbf{v}})$. Then $\theta(t, \mathbf{x})$ solves both

$$
\begin{array}{ll}
\frac{\partial}{\partial t} \theta^{(i)}(t, \mathbf{x})=-\mathbf{v}(\mathbf{x}) \cdot \nabla \theta^{(i)}(t, \mathbf{x})+\kappa \Delta \theta^{(i)}(t, \mathbf{x}) & , \quad \theta^{(i)}(0, \mathbf{x})=\theta_{0}^{(i)}(\mathbf{x}) \\
\frac{\partial}{\partial t} \theta^{(i)}(t, \mathbf{x})=-\tilde{\mathbf{v}}(\mathbf{x}) \cdot \nabla \theta^{(i)}(t, \mathbf{x})+\kappa \Delta \theta^{(i)}(t, \mathbf{x}) \quad, & \theta^{(i)}(0, \mathbf{x})=\theta_{0}^{(i)}(\mathbf{x})
\end{array}
$$

for $i=1,2$ and all $t, \mathbf{x}$. Subtraction yields

$$
0=(\tilde{\mathbf{v}}(\mathbf{x})-\mathbf{v}(\mathbf{x})) \cdot \nabla \theta^{(i)}(t, \mathbf{x})
$$


for $i=1,2$ and all $t, \mathbf{x}$. In particular,

$$
0=(\tilde{\mathbf{v}}(\mathbf{x})-\mathbf{v}(\mathbf{x})) \cdot \nabla \theta_{0}^{(i)}(\mathbf{x})
$$

for $i=1,2$ and all $\mathbf{x}$. However, by Assumption 4.4, $\nabla \theta_{0}^{(1)}(\mathbf{x}), \nabla \theta_{0}^{(2)}(\mathbf{x})$ span $\mathbb{R}^{2}$ at almost all $\mathbf{x}$. Therefore $\tilde{\mathbf{v}}(\mathbf{x})=\mathbf{v}(\mathbf{x})$ for almost all $\mathbf{x}$. Therefore $\|\mathbf{v}-\tilde{\mathbf{v}}\|_{H}=0$.

Lemma 4.9. Let $Y, Z$ be metric spaces. Suppose that $B \subset Y$ is compact. Let $f: Y \rightarrow Z$ be injective and continuous. Then $f^{-1}: f(B) \rightarrow Y$ is continuous. (Here, we denote $f(B):=$ $\{z \in Z: \exists y \in B$ such thatf $(y)=z\}$.)

Proof. Let $z_{n}, z \in f(B)$ such that $z_{n} \rightarrow z$. Define $y_{n}, y \in B$ such that $y_{n}=f^{-1}\left(z_{n}\right)$ and $y=f^{-1}(z)$. Since $B$ compact, there exists a subsequence $y_{n^{\prime}}$ that converges in $B$; denote this limit $\tilde{y} \in B$. Then since $f$ continuous, $f\left(y_{n^{\prime}}\right) \rightarrow f(\tilde{y})$. But by definition, we also have $f\left(y_{n^{\prime}}\right)=z_{n^{\prime}} \rightarrow z=f(y)$. So $f(\tilde{y})=f(y)$. Since $f$ injective, $y=\tilde{y}$, i.e., $f^{-1}\left(z_{n}\right) \rightarrow f^{-1}(z)$. So $f^{-1}: f(B) \rightarrow Y$ is continuous.

Corollary $4.10\left(\tilde{\mathcal{S}}^{-1}\right.$ continuous $)$. Let $\tilde{\mathcal{S}}: H \rightarrow L^{2}\left([0, T] \times \mathbb{T}^{2}\right)^{2}$ be the paired solution map given in Definition 4.6 with initial conditions meeting Assumption 4.4. Then for any $r>0$ and $\mathbf{v}_{0} \in V, \tilde{\mathcal{S}}^{-1}: \tilde{\mathcal{S}}\left(B_{V}^{r}\left(\mathbf{v}_{0}\right)\right) \rightarrow H$ is continuous.

Proof. We have $\tilde{\mathcal{S}}: H \rightarrow L^{2}\left([0, T] \times \mathbb{T}^{2}\right)^{2}$ continuous by Corollary 4.7 and injective by Lemma 4.8. We also have $B_{V}^{r}\left(\mathbf{v}_{0}\right)$ compact in $H$ by Lemma 4.2. Therefore, $\tilde{\mathcal{S}}^{-1}: \tilde{\mathcal{S}}\left(B_{V}^{r}\left(\mathbf{v}_{0}\right)\right) \rightarrow$ $H$ is continuous by Lemma 4.9 .

Corollary 4.11. Let $r>0$. For all $\epsilon>0$, there exists a $\delta>0$ such that

$$
\left\{\mathbf{v} \in H:\left\|\tilde{\mathcal{S}}(\mathbf{v})-\tilde{\mathcal{S}}\left(\mathbf{v}^{\star}\right)\right\|_{L^{2}\left([0, T] \times \mathbb{T}^{2}\right)^{2}}<\delta\right\} \cap B_{V}^{r}\left(\mathbf{v}^{\star}\right) \subset B_{H}^{\epsilon}\left(\mathbf{v}^{\star}\right)
$$




\subsection{Convergence of Posterior to the True Scalar Field}

In this section, we show that the Bayesian posterior measure for $N$ point observations $\mu_{\mathcal{Y}}^{N}$ converges to background flows that match the true scalar field $\theta\left(\mathbf{v}^{\star}\right)$. We proceed via a series of lemmas. In Lemma 4.12, we construct a useful decomposition of the log-likelihood, which we then extend to the multiple-initial condition case in Lemma 4.13. Convergence is then shown to be uniform in probability in Corollary 4.15. In Lemma 4.21, we then use this decomposition to show that, as the number of observations grows, the observation noise is averaged out, the effect of the prior diminishes, and the Bayesian posterior focuses on background flows for which $\theta(\mathbf{v})=\theta\left(\mathbf{v}^{\star}\right)$. In proving this result, we apply Assumption 4.17 and Assumption 4.18 to the prior measure $\mu_{0}$.

We begin by showing that for large $N$, the growth in the log-likelihood for a vector field $\mathbf{v}$ (normalized by $\frac{1}{N}$ ) can be written in terms of the observation error and the difference between $\mathbf{v}$ and $\mathbf{v}^{\star}$.

Lemma 4.12. Let $\theta(t, \mathbf{x}, \mathbf{v})$ denote the solution of the advection-diffusion equation (1.1):

$$
\frac{\partial}{\partial t} \theta(t, \mathbf{x}, \mathbf{v})=-\mathbf{v}(\mathbf{x}) \cdot \nabla \theta(t, \mathbf{x}, \mathbf{v})+\kappa \Delta \theta(t, \mathbf{x}, \mathbf{v}) \quad, \quad \theta(0, \mathbf{x}, \mathbf{v})=\theta_{0}(\mathbf{x})
$$

Let $\left\{\left(t_{j}, \mathbf{x}_{j}\right)\right\}_{j=1}^{\infty}$ be a sequence of observation points uniformly distributed in $[0, T] \times \mathbb{T}^{2}$. Denote the associated data by $\left\{\mathcal{Y}_{j}\right\}_{j=1}^{\infty}$, where

$$
\mathcal{Y}_{j}=\theta\left(t_{j}, \mathbf{x}_{j}, \mathbf{v}^{\star}\right)+\eta_{j}
$$

for i.i.d. observational noise $\eta_{j} \sim N\left(0, \sigma_{\eta}^{2}\right)$ and some true vector field $\mathbf{v}^{\star}$. 
Then for any background flow $\mathbf{v}$,

$$
\lim _{N \rightarrow \infty} \frac{1}{N} \sum_{j=1}^{N}\left(\mathcal{Y}_{j}-\theta\left(t_{j}, \mathbf{x}_{j}, \mathbf{v}\right)\right)^{2}=\sigma_{\eta}^{2}+\frac{1}{T}\left\|\theta\left(\mathbf{v}^{\star}\right)-\theta(\mathbf{v})\right\|_{L^{2}\left([0, T] \times \mathbb{T}^{2}\right)}^{2} \quad \text { a.s. }
$$

Proof. Denote $\theta_{j}=\theta\left(t_{j}, \mathbf{x}_{j}, \mathbf{v}\right)$ and $\theta_{j}^{\star}=\theta\left(t_{j}, \mathbf{x}_{j}, \mathbf{v}^{\star}\right)$.

$$
\begin{aligned}
\lim _{N \rightarrow \infty} \frac{1}{N} \sum_{j=1}^{N}\left(\mathcal{Y}_{j}-\theta_{j}\right)^{2} & =\lim _{N \rightarrow \infty} \frac{1}{N} \sum_{j=1}^{N}\left(\eta_{j}+\theta_{j}^{\star}-\theta_{j}\right)^{2} \\
& =\lim _{N \rightarrow \infty} \frac{1}{N} \sum_{j=1}^{N} \eta_{j}^{2}+2 \lim _{N \rightarrow \infty} \frac{1}{N} \sum_{j=1}^{N} \eta_{j}\left(\theta_{j}^{\star}-\theta_{j}\right)+\lim _{N \rightarrow \infty} \frac{1}{N} \sum_{j=1}^{N}\left(\theta_{j}^{\star}-\theta_{j}\right)^{2} \\
& \equiv T_{1}+T_{2}+T_{3}
\end{aligned}
$$

We will now focus on each of three terms on the right hand side. For the first term, the law of large numbers yields

$$
T_{1}=\lim _{N \rightarrow \infty} \frac{1}{N} \sum_{j=1}^{N} \eta_{j}^{2}=\mathbb{E} \eta_{j}^{2}=\sigma_{\eta}^{2} \quad \text { a.s. }
$$

For the second term, since $\left\{\eta_{j}\right\}$ and $\left\{t_{j}, \mathbf{x}_{j}\right\}$ are independent, so are $\left\{\eta_{j}\right\}$ and $\left\{\theta\left(t_{j}, \mathbf{x}_{j}, \mathbf{v}^{\star}\right)-\theta\left(t_{j}, \mathbf{x}_{j}, \mathbf{v}\right)\right\}$. Then, again using the law of large numbers,

$$
T_{2}=\lim _{N \rightarrow \infty} \frac{1}{N} \sum_{j=1}^{N} \eta_{j}\left(\theta_{j}^{\star}-\theta_{j}\right)=\mathbb{E}\left[\eta_{j}\left(\theta_{j}^{\star}-\theta_{j}\right)\right]=\mathbb{E} \eta_{j} \mathbb{E}\left(\theta_{j}^{\star}-\theta_{j}\right)=0 \quad \text { a.s. }
$$

since $\mathbb{E} \eta_{j}=0$.

Finally, for the third term, Monte Carlo integration yields

$$
T_{3}=\lim _{N \rightarrow \infty} \frac{1}{N} \sum_{j=1}^{N}\left(\theta_{j}^{\star}-\theta_{j}\right)^{2}=\frac{1}{T} \int_{0}^{T} \int_{\mathbb{T}^{2}}\left(\theta^{\star}-\theta\right)^{2}=\frac{1}{T}\left\|\theta^{\star}-\theta\right\|_{L^{2}\left([0, T] \times \mathbb{T}^{2}\right)}^{2} \quad \text { a.s. }
$$


Assembling (4.16), (4.17), and (4.18) yields the desired result (4.14).

In the next lemma, we extend Lemma 4.12 to observations for multiple initial conditions outlined in Definition 4.5.

Lemma 4.13. Let $\left\{\left(t_{j}, \mathbf{x}_{j}\right)\right\}_{j=1}^{\infty}$ be a sequence of observation points uniformly distributed in $[0, T] \times \mathbb{T}^{2}$. Denote the associated data by $\left\{\mathcal{Y}_{j}\right\}_{j=1}^{\infty}$, where

$$
\mathcal{Y}_{j}=\mathcal{G}_{j}\left(\mathbf{v}^{\star}\right)+\eta_{j}
$$

for i.i.d. observational noise $\eta_{j} \sim N\left(0, \sigma_{\eta}^{2}\right)$, some true vector field $\mathbf{v}^{\star}$, and parameter-toobservable (forward) map $\mathcal{G}_{j}$ given by Definition 4.5 .

Then

$$
\lim _{N \rightarrow \infty} \frac{1}{N} \sum_{j=1}^{N}\left(\mathcal{Y}_{j}-\mathcal{G}_{j}(\mathbf{v})\right)^{2}=\sigma_{\eta}^{2}+\frac{1}{2 T}\left\|\tilde{\mathcal{S}}\left(\mathbf{v}^{\star}\right)-\tilde{\mathcal{S}}(\mathbf{v})\right\|_{L^{2}\left([0, T] \times \mathbb{T}^{2}\right)^{2}}^{2} \quad \text { a.s. }
$$

where $\tilde{\mathcal{S}}$ is the paired solution operator defined in Definition 4.6. 
Proof. By Lemma 4.12

$$
\begin{aligned}
& \lim _{N \rightarrow \infty} \frac{1}{N} \sum_{j=1}^{N}\left(\mathcal{Y}_{j}-\mathcal{G}_{j}(\mathbf{v})\right)^{2}=\lim _{N \rightarrow \infty} \frac{1}{2 N} \sum_{j=1}^{2 N}\left(\mathcal{Y}_{j}-\mathcal{G}_{j}(\mathbf{v})\right)^{2} \\
&=\lim _{N \rightarrow \infty} \frac{1}{2 N}\left[\sum_{j=1}^{N}\left(\mathcal{Y}_{2 j-1}-\mathcal{G}_{2 j-1}(\mathbf{v})\right)^{2}+\sum_{j=1}^{N}\left(\mathcal{Y}_{2 j}-\mathcal{G}_{2 j}(\mathbf{v})\right)^{2}\right] \\
&=\frac{1}{2} \lim _{N \rightarrow \infty} \frac{1}{N} \sum_{j=1}^{N}\left(\mathcal{Y}_{j}-\theta^{(1)}\left(t_{j}, \mathbf{x}_{j}, \mathbf{v}\right)\right)^{2}+\frac{1}{2} \lim _{N \rightarrow \infty} \frac{1}{N} \sum_{j=1}^{N}\left(\mathcal{Y}_{j}-\theta^{(2)}\left(t_{j}, \mathbf{x}_{j}, \mathbf{v}\right)\right)^{2} \\
&=\frac{1}{2}\left(\sigma_{\eta}^{2}+\frac{1}{T}\left\|\theta^{(1)}\left(\mathbf{v}^{\star}\right)-\theta^{(1)}(\mathbf{v})\right\|_{L^{2}\left([0, T] \times \mathbb{T}^{2}\right)}^{2}\right) \\
&+\frac{1}{2}\left(\sigma_{\eta}^{2}+\frac{1}{T}\left\|\theta^{(2)}\left(\mathbf{v}^{\star}\right)-\theta^{(2)}(\mathbf{v})\right\|_{L^{2}\left([0, T] \times \mathbb{T}^{2}\right)}^{2}\right) \\
&= \sigma_{\eta}^{2}+\frac{1}{2 T}\left\|\tilde{\mathcal{S}}\left(\mathbf{v}^{\star}\right)-\tilde{\mathcal{S}}(\mathbf{v})\right\|_{L^{2}\left([0, T] \times \mathbb{T}^{2}\right)^{2}}^{2}
\end{aligned}
$$

a.s., giving (4.20).

It turns out that the convergence in (4.14) and (4.20) is uniform in $\mathbf{v}$ for $\mathbf{v} \in B_{V}^{r}\left(\mathbf{v}^{\star}\right)$. To show this, we will need a version of the uniform law of large numbers (see also [66]), which we now present.

Lemma 4.14 (Uniform Law of Large Numbers). Let $X$ be a metric space with $B \subset X$ compact and $f: \mathbb{R}^{n} \times X \rightarrow \mathbb{R}$ measurable. Let $\left\{Z_{j}\right\}_{j=1}^{\infty} \in \mathbb{R}^{n}$ be an i.i.d. sequence of random variables. Assume that $\mathbb{E} f(Z, x)^{2}<\infty$ for all $x \in B$ and that there exists a deterministic function $d: \mathbb{R}^{n} \rightarrow \mathbb{R}^{+}$with $\mathbb{E} d(Z)^{2}<\infty$ such that for all $\epsilon>0$ and $x \in B$, there exists a $\delta>0$ such that

$$
\|x-\tilde{x}\|<\delta(x) \Longrightarrow|f(Z, x)-f(Z, \tilde{x})| \leq d(Z) \epsilon
$$

for all Z. Then

$$
\lim _{N \rightarrow \infty} \sup _{x \in B}\left|\frac{1}{N} \sum_{j=1}^{N} f\left(Z_{j}, x\right)-\mathbb{E} f(Z, x)\right|=0 \quad \text { a.s. }
$$


Proof. Note that since $d$ is positive, $\mathbb{E} d(Z)=0$ implies that $d(Z)$ is almost surely 0 , in which case $f$ is almost surely constant and the result is trivial. We therefore focus on the case where $\mathbb{E} d(Z) \neq 0$.

Define $g(Z, x):=f(Z, x)-\mathbb{E} f(Z, x)$. Then by assumptions on $f, \mathbb{E} g(\cdot, x)^{2}<\infty$. Note also that

$$
|f(Z, x)-f(Z, \tilde{x})| \leq d(Z) \epsilon \Longrightarrow|g(Z, x)-g(Z, \tilde{x})| \leq[d(Z)+\mathbb{E} d(Z)] \epsilon
$$

Let $\epsilon>0$. Then by (4.22) and (4.24), for each $x$ there exists a $\delta(x)>0$ such that $\|\tilde{x}-x\|_{X}<$ $\delta(x)$ implies $|g(Z, \tilde{x})-g(Z, x)|<\frac{d(Z)+\mathbb{E} d(Z)}{2 \mathbb{E} d} \epsilon$. Let $B^{\delta(x)}(x)=\left\{\tilde{x}:\|\tilde{x}-x\|_{X}<\delta(x)\right\}$ and note that $\cup_{x \in B} B^{\delta(x)}(x)=B$. Then since $B$ is compact, there exists a finite subcovering $\left\{B^{\delta_{i}}\left(x_{i}\right)\right\}_{i=1}^{m}$ such that

$$
\cup_{i=1}^{m} B^{\delta_{i}}\left(x_{i}\right)=B
$$

Let $x \in B$ and let $i$ be the index such that $x \in B^{\delta_{i}}\left(x_{i}\right)$. Then

$$
\begin{aligned}
\left|\frac{1}{N} \sum_{j=1}^{N} g\left(Z_{j}, x\right)\right| & =\left|\frac{1}{N} \sum_{j=1}^{N} g\left(Z_{j}, x\right)-g\left(Z_{j}, x_{i}\right)+g\left(Z_{j}, x_{i}\right)\right| \\
& \leq\left|\frac{1}{N} \sum_{j=1}^{N} g\left(Z_{j}, x\right)-g\left(Z_{j}, x_{i}\right)\right|+\left|\frac{1}{N} \sum_{j=1}^{N} g\left(Z_{j}, x_{i}\right)\right| \\
& \leq \frac{\epsilon}{2 \mathbb{E} d}\left|\frac{1}{N} \sum_{j=1}^{N} d\left(Z_{j}\right)+\mathbb{E} d(Z)\right|+\left|\frac{1}{N} \sum_{j=1}^{N} g\left(Z_{j}, x_{i}\right)\right|
\end{aligned}
$$


Taking the supremum over $x$ and using the subcovering yields

$$
\begin{aligned}
\sup _{x \in B} \mid & \left|\frac{1}{N} \sum_{j=1}^{N} g\left(Z_{j}, x\right)\right| \\
& \leq \max _{i=1, \ldots, m} \sup _{x \in B^{\delta_{i}\left(x_{i}\right)}}\left[\frac{\epsilon}{2 \mathbb{E} d}\left|\frac{1}{N} \sum_{j=1}^{N} d\left(Z_{j}\right)+\mathbb{E} d(Z)\right|+\max _{i=1, \ldots, m}\left|\frac{1}{N} \sum_{j=1}^{N} g\left(Z_{j}, x_{i}\right)\right|\right] \\
& \leq \frac{\epsilon}{2 \mathbb{E} d}\left|\frac{1}{N} \sum_{j=1}^{N} d\left(Z_{j}\right)+\mathbb{E} d(Z)\right|+\max _{i=1, \ldots, m}\left|\frac{1}{N} \sum_{j=1}^{N} g\left(Z_{j}, x_{i}\right)\right| .
\end{aligned}
$$

Then the Strong Law of Large Numbers gives

$$
\begin{aligned}
\limsup _{N \rightarrow \infty} & {\left[\sup _{x \in B}\left|\frac{1}{N} \sum_{j=1}^{N} g\left(Z_{j}, x\right)\right|\right] } \\
& \leq \limsup _{N \rightarrow \infty} \frac{\epsilon}{2 \mathbb{E} d}\left|\frac{1}{N} \sum_{j=1}^{N} d\left(Z_{j}\right)+\mathbb{E} d(Z)\right|+\max _{i=1, \ldots, m}\left|\frac{1}{N} \sum_{j=1}^{N} g\left(Z_{j}, x_{i}\right)\right| \\
& \leq \epsilon \frac{2 \mathbb{E} d}{2 \mathbb{E} d}+\max _{i=1, \ldots, m} \mathbb{E} g\left(Z, x_{i}\right)=\epsilon \quad \text { a.s. }
\end{aligned}
$$

Thus, we have

$$
\Omega_{\epsilon}:=\left\{\lim _{N \rightarrow \infty} \sup _{x \in B}\left|\frac{1}{N} \sum_{j=1}^{N} g\left(Z_{j}, x\right)\right|<\epsilon\right\}
$$

has probability 1 for all $\epsilon>0$. Then taking $\Omega_{0}=\cap_{k=1}^{\infty} \Omega_{\frac{1}{k}}$ and invoking the continuity of measures,

$$
\mathbb{P}\left\{\Omega_{0}\right\}=\mathbb{P}\left\{\lim _{N \rightarrow \infty} \sup _{x \in B}\left|\frac{1}{N} \sum_{j=1}^{N} g\left(Z_{j}, x\right)\right|=0\right\}=\lim _{K \rightarrow \infty} \mathbb{P}\left\{\cap_{k=1}^{K} \Omega_{\frac{1}{k}}\right\}=1
$$

Corollary 4.15. Let $r>0$. The convergence in (4.20) is uniform for $\mathbf{v} \in B_{V}^{r}\left(\mathbf{v}^{\star}\right)$. That is,

$$
\sup _{\mathbf{v} \in B_{V}^{r}\left(\mathbf{v}^{\star}\right)}\left|\frac{1}{N} \sum_{j=1}^{N}\left(\mathcal{Y}_{j}-\mathcal{G}_{j}(\mathbf{v})\right)^{2}-\left(\sigma_{\eta}^{2}+\frac{1}{2 T}\left\|\tilde{\mathcal{S}}\left(\mathbf{v}^{\star}\right)-\tilde{\mathcal{S}}(\mathbf{v})\right\|_{L^{2}\left([0, T] \times \mathbb{T}^{2}\right)^{2}}^{2}\right)\right| \rightarrow 0
$$


Proof. From the proof of Lemma 4.12, we have the decomposition

$$
\begin{aligned}
\lim _{N \rightarrow \infty} \frac{1}{N} \sum_{j=1}^{N}\left(\mathcal{Y}_{j}-\theta_{j}\right)^{2} & =\lim _{N \rightarrow \infty} \frac{1}{N} \sum_{j=1}^{N} \eta_{j}^{2}+2 \lim _{N \rightarrow \infty} \frac{1}{N} \sum_{j=1}^{N} \eta_{j}\left(\theta_{j}^{\star}-\theta_{j}\right)+\lim _{N \rightarrow \infty} \frac{1}{N} \sum_{j=1}^{N}\left(\theta_{j}^{\star}-\theta_{j}\right)^{2} \\
& =T_{1}+T_{2}(\mathbf{v})+T_{3}(\mathbf{v})
\end{aligned}
$$

a.s., where we use the notation $\theta_{j}=\theta\left(t_{j}, \mathbf{x}_{j}, \mathbf{v}\right)$ and $\theta_{j}^{\star}=\theta\left(t_{j}, \mathbf{x}_{j}, \mathbf{v}^{\star}\right)$.

$T_{1}$ is independent of, and therefore uniform in, $\mathbf{v}$. So we focus on the terms $T_{2}$ and $T_{3}$.

$\operatorname{Term} \mathrm{T}_{2}(\mathbf{v})$ :

Denote $Z=[\eta, t, \mathbf{x}] \in \mathbb{R}^{3}$ and define

$$
f(Z, \mathbf{v})=\eta\left(\theta\left(t, \mathbf{x}, \mathbf{v}^{\star}\right)-\theta(t, \mathbf{x}, \mathbf{v})\right)
$$

Note that by assumption on $\eta, \mathbb{E} \eta^{2}=\sigma_{\eta}^{2}<\infty$. So by the maximum principle, $\mathbb{E} f(\cdot, \mathbf{v})^{2}<$ $\left\|\theta_{0}\right\|_{L^{\infty}}^{2} \mathbb{E}|\eta|^{2}<\infty$. And finally, by Corollary 3.10, for all $\epsilon>0$ there exists a $\delta$ such that

$$
\|\mathbf{v}-\tilde{\mathbf{v}}\|<\delta(\mathbf{v}) \Longrightarrow|f(Z, \mathbf{v})-f(Z, \tilde{\mathbf{v}})| \leq|\eta||\theta(t, \mathbf{x}, \mathbf{v})-\theta(t, \mathbf{x}, \tilde{\mathbf{v}})| \leq \eta \epsilon
$$

Since $B_{V}^{r}\left(\mathbf{v}^{\star}\right)$ is compact in $H$ by Lemma 4.2, Lemma 4.14 above yields

$$
\sup _{\mathbf{v} \in B_{V}^{r}\left(\mathbf{v}^{\star}\right)}\left|T_{2}(\mathbf{v})-\mathbb{E} T_{2}(\mathbf{v})\right|=\sup _{\mathbf{v} \in B_{V}^{r}\left(\mathbf{v}^{\star}\right)}\left|\frac{1}{N} \sum_{j=1}^{N} \eta_{j}\left(\theta_{j}^{\star}-\theta_{j}\right)\right| \rightarrow 0 \quad \text { a.s. }
$$

$\operatorname{Term~} \mathrm{T}_{3}(\mathbf{v})$ : 
Denote $Z=[\eta, t, \mathbf{x}] \in \mathbb{R}^{3}$ and define

$$
f(Z, \mathbf{v})=\left(\theta\left(t, \mathbf{x}, \mathbf{v}^{\star}\right)-\theta(t, \mathbf{x}, \mathbf{v})\right)^{2} .
$$

Note that by the maximum principle, $\mathbb{E} f(\cdot, \mathbf{v})^{2}<\left\|\theta_{0}\right\|_{L^{\infty}}^{4}<\infty$. Also, by Corollary 3.10, for all $\epsilon>0$ there exists a $\delta$ such that

$$
\|\mathbf{v}-\tilde{\mathbf{v}}\|<\delta(\mathbf{v}) \Longrightarrow|f(Z, \mathbf{v})-f(Z, \tilde{\mathbf{v}})|=|\theta(t, \mathbf{x}, \mathbf{v})-\theta(t, \mathbf{x}, \tilde{\mathbf{v}})|^{2} \leq \epsilon
$$

Since $B_{V}^{r}\left(\mathbf{v}^{\star}\right)$ is compact in $H$ by Lemma 4.2, Lemma 4.14 gives uniform convergence:

$$
\sup _{\mathbf{v} \in B_{V}^{r}\left(\mathbf{v}^{\star}\right)}\left|T_{3}(\mathbf{v})-\mathbb{E} T_{3}(\mathbf{v})\right|=\sup _{\mathbf{v} \in B_{V}^{r}\left(\mathbf{v}^{\star}\right)}\left|\frac{1}{N} \sum_{j=1}^{N}\left(\theta_{j}^{\star}-\theta_{j}\right)^{2}-\frac{1}{T}\left\|\theta^{\star}-\theta\right\|_{L^{2}\left([0, T] \times \mathbb{T}^{2}\right)}^{2}\right| \rightarrow 0 \quad \text { a.s. }
$$

Assembling the results for $\theta_{0}^{(1)}, \theta_{0}^{(2)}$ (as in the proof of Lemma 4.13) yields the desired result.

We now turn our attention to showing that the posterior converges to a Dirac measure. In what follows, it is useful to define the set of $\mathbf{v}$ such that $\theta(\mathbf{v})$ is close to $\theta\left(\mathbf{v}^{\star}\right)$ :

Definition $4.16\left(H_{\delta}\right)$. Denote the set $H_{\delta}$ by:

$$
H_{\delta}=\left\{\mathbf{v} \in H:\left\|\tilde{\mathcal{S}}\left(\mathbf{v}^{\star}\right)-\tilde{\mathcal{S}}(\mathbf{v})\right\|_{L^{2}\left([0, T] \times \mathbb{T}^{2}\right)^{2}}<\delta\right\}
$$

We now make two assumptions about the prior measure, $\mu_{0}$. The first is that it cannot "rule out" the true background flow $\mathbf{v}^{\star}$. The second is that it cannot place a large weight on background flows with low regularity. 
Assumption 4.17 (Support Condition on $\mu_{0}$ ). For any $r>0, \mu_{0}\left(B_{V}^{r}\left(\mathbf{v}^{\star}\right)\right)>0$.

Assumption 4.18 (Tail Condition on $\mu_{0}$ ). The prior measure $\mu_{0}$ satisfies one of

a) There exists an $r>0$ such that $\mu_{0}\left(B_{V}^{r}(\mathbf{0})\right)=1$

b) $\mu_{0}$ is such that there exists an $f: \mathbb{R}^{+} \rightarrow \mathbb{R}^{+}$such that $f$ is monotone increasing with $\lim _{r \rightarrow \infty} f(r)=\infty$ and

$$
\sup _{N} \int_{H} f\left(\|\mathbf{v}\|_{V}\right) \mu_{\mathcal{Y}}^{N}(d \mathbf{v})<\infty \quad \text { a.s. }
$$

Remark 4.19. Note that Assumption 4.18(a) implies Assumption 4.18(b). The general implications of Assumption 4.18(b) for $\mu_{0}$ are not immediately clear and will be investigated in more detail in future work. However, letting $f\left(\|\mathbf{v}\|_{V}\right)=\|\mathbf{v}\|_{V}$ we can show that this assumption will be satisfied by any combination of prior measure $\mu_{0}$ and data $\mathcal{Y}$ such that the expected value of the energy in $\mathbf{v}$ (measured in $V$ ), according to the posterior measure, is finite. This should be true for most observations and a wide class of prior measures with tails that converge sufficiently fast.

Lemma 4.20. Let $\mathbf{v}^{\star}$ satisfy Assumption 4.3 and $\mu_{0}$ satisfy Assumption 4.17 and Assumption 4.18. Then for any $\delta, \epsilon>0$, there exists an $R>0$ so that both

$$
\mu_{0}\left(H_{\delta} \cap B_{V}^{R}\left(\mathbf{v}^{\star}\right)\right)>0 \quad \text { and } \quad \mu_{\mathcal{Y}}^{N}\left(\left(B_{V}^{R}\left(\mathbf{v}^{\star}\right)\right)^{c}\right)<\epsilon .
$$

Proof. Let $\delta>0$ and $\epsilon>0$.

By Corollary 4.7, there exists an $r>0$ such that $B_{V}^{r}\left(\mathbf{v}^{\star}\right) \subset H_{\delta}$. Then for any $R>r$, $B_{V}^{r}\left(\mathbf{v}^{\star}\right) \subset H_{\delta} \cap B_{V}^{R}\left(\mathbf{v}^{\star}\right)$. By Assumption 4.17, $\mu_{0}\left(H_{\delta} \cap B_{V}^{R}\left(\mathbf{v}^{\star}\right)\right)>0$. 
If $\mu_{0}$ satisfies Assumption $4.18(\mathrm{a})$, let $R>r$ such that $\mu_{0}\left(B_{V}^{R}(\mathbf{0})\right)=1$, i.e., $\mu_{\mathcal{Y}}^{N}\left(\left(B_{V}^{R}(\mathbf{0})\right)^{c}\right)=$ 0.

If $\mu_{0}$ satisfies Assumption 4.18(b), let $R>r$ such that

$$
\frac{1}{f(R)} \sup _{N} \int_{H} f\left(\|\mathbf{v}\|_{V}\right) \mu_{\mathcal{Y}}^{N}(d \mathbf{v})<\epsilon .
$$

Then

$$
\begin{aligned}
\mu_{\mathcal{Y}}^{N}\left(\left(B_{V}^{R}(\mathbf{0})\right)^{c}\right) & =\int_{\left(B_{V}^{R}(\mathbf{0})\right)^{c}} \mu_{\mathcal{Y}}^{N}(d \mathbf{v})=\int_{\left(B_{V}^{R}(\mathbf{0})\right)^{c}} \frac{f\left(\|\mathbf{v}\|_{V}\right)}{f\left(\|\mathbf{v}\|_{V}\right)} \mu_{\mathcal{Y}}^{N}(d \mathbf{v}) \leq \int_{\left(B_{V}^{R}(\mathbf{0})\right)^{c}} \frac{f\left(\|\mathbf{v}\|_{V}\right)}{f(R)} \mu_{\mathcal{Y}}^{N}(d \mathbf{v}) \\
& \leq \frac{1}{f(R)} \int_{\left(B_{V}^{R}(\mathbf{0})\right)^{c}} f\left(\|\mathbf{v}\|_{V}\right) \mu_{\mathcal{Y}}^{N}(d \mathbf{v}) \leq \frac{1}{f(R)} \int_{H} f\left(\|\mathbf{v}\|_{V}\right) \mu_{\mathcal{Y}}^{N}(d \mathbf{v})<\epsilon
\end{aligned}
$$

Thus, for either of the conditions in Assumption 4.18, $\mu_{\mathcal{Y}}^{N}\left(\left(B_{V}^{R}(\mathbf{0})\right)^{c}\right)<\epsilon$. Selecting $\tilde{R}>$ $R+\left\|\mathbf{v}^{\star}\right\|_{V}$ ensures that $\left(B_{V}^{\tilde{R}}\left(\mathbf{v}^{\star}\right)\right)^{c} \subset\left(B_{V}^{R}(\mathbf{0})\right)^{c}$, and so $\mu_{\mathcal{Y}}^{N}\left(\left(B_{V}^{\tilde{R}}\left(\mathbf{v}^{\star}\right)\right)^{c}\right)<\epsilon$.

We now show that as the number of observations increases, the Bayesian posterior converges on the vector fields for which $\theta(\mathbf{v})$ is close to $\theta\left(\mathbf{v}^{\star}\right)$.

Lemma 4.21 (Identification of true $\theta)$. Let $\left\{\left(t_{j}, \mathbf{x}_{j}\right)\right\}, \mathcal{G}_{j}$, and $\left\{\mathcal{Y}_{j}\right\}$ be observation points, forward map, and observations, respectively, satisfying the conditions of Lemma 4.13. Let $\mu_{\mathcal{Y}}^{N}$ be the posterior measure for $N$ observations, given by

$$
\mu_{\mathcal{Y}}^{N}(d \mathbf{v})=\frac{1}{Z} \exp \left[-\frac{1}{2 \sigma_{\eta}^{2}} \sum_{j=1}^{N}\left(\mathcal{Y}_{j}-\mathcal{G}_{j}(\mathbf{v})\right)^{2}\right] \mu_{0}(d \mathbf{v})
$$

where $Z$ is the normalization

$$
Z=\int_{H} \exp \left[-\frac{1}{2 \sigma_{\eta}^{2}} \sum_{j=1}^{N}\left(\mathcal{Y}_{j}-\mathcal{G}_{j}(\mathbf{v})\right)^{2}\right] \mu_{0}(d \mathbf{v})
$$


Suppose that the true background flow $\mathbf{v}^{\star}$ satisfies Assumption 4.3 and the prior measure $\mu_{0}$ satisfies Assumption 4.17 and Assumption 4.18. Then for any $\delta>0$,

$$
\mu_{\mathcal{Y}}^{N}\left(H_{\delta}\right) \rightarrow 1 \quad \text { a.s. }
$$

Proof. Let $\delta, \epsilon>0$. As in Lemma 4.20, select $R>0$ so that both

$$
\mu_{0}\left(H_{\delta} \cap B_{V}^{R}\left(\mathbf{v}^{\star}\right)\right)>0 \quad \text { and } \quad \mu_{\mathcal{Y}}^{N}\left(\left(B_{V}^{R}\left(\mathbf{v}^{\star}\right)\right)^{c}\right)<\frac{\epsilon}{2} .
$$

By Corollary 4.15, there exists an $N_{1}$ such that for all $N>N_{1}$ and $\mathbf{v} \in B_{V}^{R}\left(\mathbf{v}^{\star}\right)$,

$$
\left|\frac{1}{N} \sum_{j=1}^{N}\left(\mathcal{Y}_{j}-\mathcal{G}_{j}(\mathbf{v})\right)^{2}-\left(\sigma_{\eta}^{2}+\frac{1}{2 T}\left\|\tilde{\mathcal{S}}\left(\mathbf{v}^{\star}\right)-\tilde{\mathcal{S}}(\mathbf{v})\right\|_{L^{2}\left([0, T] \times \mathbb{T}^{2}\right)^{2}}^{2}\right)\right|<\frac{\delta^{2}}{8 T} \quad \text { a.s. }
$$

Then for $\mathbf{v} \in H_{\frac{\delta}{2}} \cap B_{V}^{R}\left(\mathbf{v}^{\star}\right)$ and $N>N_{1}$, we have

$$
\frac{1}{N} \sum_{j=1}^{N}\left(\mathcal{Y}_{j}-\mathcal{G}_{j}(\mathbf{v})\right)^{2}<\sigma_{\eta}^{2}+\frac{1}{2 T}\left\|\tilde{\mathcal{S}}\left(\mathbf{v}^{\star}\right)-\tilde{\mathcal{S}}(\mathbf{v})\right\|_{L^{2}\left([0, T] \times \mathbb{T}^{2}\right)^{2}}^{2}+\frac{\delta^{2}}{8 T}<\sigma_{\eta}^{2}+\frac{\delta^{2}}{4 T} \quad \text { a.s. }
$$

Similarly, for $\mathbf{v} \in H_{\delta}^{c} \cap B_{V}^{R}\left(\mathbf{v}^{\star}\right)$ and $N>N_{1}$, we have

$$
\frac{1}{N} \sum_{j=1}^{N}\left(\mathcal{Y}_{j}-\mathcal{G}_{j}(\mathbf{v})\right)^{2}>\sigma_{\eta}^{2}+\frac{1}{2 T}\left\|\tilde{\mathcal{S}}\left(\mathbf{v}^{\star}\right)-\tilde{\mathcal{S}}(\mathbf{v})\right\|_{L^{2}\left([0, T] \times \mathbb{T}^{2}\right)^{2}}^{2}-\frac{\delta^{2}}{8 T} \geq \sigma_{\eta}^{2}+\frac{3 \delta^{2}}{8 T} \quad \text { a.s. }
$$

Now, leveraging $\mu_{0}\left(H_{\frac{\delta}{2}} \cap B_{V}^{R}\left(\mathbf{v}^{\star}\right)\right)>0$, we choose $N_{2}$ such that

$$
\frac{1}{\mu_{0}\left(H_{\frac{\delta}{2}} \cap B_{V}^{R}\left(\mathbf{v}^{\star}\right)\right)} \exp \left[-\frac{\delta^{2}}{16 T \sigma_{\eta}^{2}} N_{2}\right]<\frac{\epsilon}{2}
$$


Then for all $N>N_{1}, N_{2}$, we have

$$
\begin{aligned}
\mu_{\mathcal{Y}}^{N}\left(H_{\delta}^{c} \cap B_{V}^{R}\left(\mathbf{v}^{\star}\right)\right)= & \frac{\int_{H_{\delta}^{c} \cap B_{V}^{R}\left(\mathbf{v}^{\star}\right)} \exp \left[-\frac{1}{2 \sigma_{\eta}^{2}} \sum_{j=1}^{N}\left(\mathcal{Y}_{j}-\mathcal{G}_{j}(\mathbf{v})\right)^{2}\right] \mu_{0}(d \mathbf{v})}{\int_{H \cap B_{V}^{R}\left(\mathbf{v}^{\star}\right)} \exp \left[-\frac{1}{2 \sigma_{\eta}^{2}} \sum_{j=1}^{N}\left(\mathcal{Y}_{j}-\mathcal{G}_{j}(\mathbf{v})\right)^{2}\right] \mu_{0}(d \mathbf{v})} \\
\leq & \frac{\int_{H_{\delta}^{c} \cap B_{V}^{R}\left(\mathbf{v}^{\star}\right)} \exp \left[-\frac{1}{2 \sigma_{\eta}^{2}} \sum_{j=1}^{N}\left(\mathcal{Y}_{j}-\mathcal{G}_{j}(\mathbf{v})\right)^{2}\right] \mu_{0}(d \mathbf{v})}{\int_{H_{\frac{\delta}{2}} \cap B_{V}^{R}\left(\mathbf{v}^{\star}\right)} \exp \left[-\frac{1}{2 \sigma_{\eta}^{2}} \sum_{j=1}^{N}\left(\mathcal{Y}_{j}-\mathcal{G}_{j}(\mathbf{v})\right)^{2}\right] \mu_{0}(d \mathbf{v})} \\
& <\frac{\int_{H_{\delta}^{c} \cap B_{V}^{R}\left(\mathbf{v}^{\star}\right)} \exp \left[-\frac{1}{2 \sigma_{\eta}^{2}} N\left(\sigma_{\eta}^{2}+\frac{3 \delta^{2}}{8 T}\right)\right] \mu_{0}(d \mathbf{v})}{\int_{H_{\frac{\delta}{2}} \cap B_{V}^{R}\left(\mathbf{v}^{\star}\right)} \exp \left[-\frac{1}{2 \sigma_{\eta}^{2}} N\left(\sigma_{\eta}^{2}+\frac{\delta^{2}}{4 T}\right)\right] \mu_{0}(d \mathbf{v})} \\
& =\frac{\exp \left[-\frac{1}{2 \sigma_{\eta}^{2}} N\left(\sigma_{\eta}^{2}+\frac{3 \delta^{2}}{8 T}\right)\right] \int_{H_{\delta}^{c} \cap B_{V}^{R}\left(\mathbf{v}^{\star}\right)} \mu_{0}(d \mathbf{v})}{\exp \left[-\frac{1}{2 \sigma_{\eta}^{2}} N\left(\sigma_{\eta}^{2}+\frac{\delta^{2}}{4 T}\right)\right] \int_{H_{\delta}} \cap B_{V}^{R}\left(\mathbf{v}^{\star}\right)} \mu_{0}(d \mathbf{v}) \\
= & \exp \left[-\frac{N \delta^{2}}{16 T \sigma_{\eta}^{2}}\right] \frac{\mu_{0}\left(H_{\delta}^{c} \cap B_{V}^{R}\left(\mathbf{v}^{\star}\right)\right)}{\mu_{0}\left(H_{\frac{\delta}{2}} \cap B_{V}^{R}\left(\mathbf{v}^{\star}\right)\right)} \\
\leq & \exp \left[-\frac{N \delta^{2}}{16 T \sigma_{\eta}^{2}}\right] \frac{1}{\mu_{0}\left(H_{\frac{\delta}{2}} \cap B_{V}^{R}\left(\mathbf{v}^{\star}\right)\right)} \\
< & \frac{\epsilon}{2} a . s .
\end{aligned}
$$

Then

$$
\mu_{\mathcal{Y}}^{N}\left(H_{\delta}^{c}\right) \leq \mu_{\mathcal{Y}}^{N}\left(H_{\delta}^{c} \cap B_{V}^{R}\left(\mathbf{v}^{\star}\right)\right)+\mu_{\mathcal{Y}}^{N}\left(\left(B_{V}^{R}\left(\mathbf{v}^{\star}\right)\right)^{c}\right)<\frac{\epsilon}{2}+\frac{\epsilon}{2}=\epsilon \quad \text { a.s. }
$$

Thus, for any $\delta>0, \mu_{\mathcal{Y}}^{N}\left(H_{\delta}\right) \rightarrow 1$ a.s.

Lemma 4.21 shows that the Bayesian posterior focuses on background flows with $\theta(\mathbf{v})=$ $\theta\left(\mathbf{v}^{\star}\right)$. Combined with the continuity of the $\theta \mapsto \mathbf{v}$ map shown earlier, we now show convergence of the posterior measure to a Dirac centered on the true vector field, $\mathbf{v}^{\star}$. 


\subsection{Convergence of Posterior Measure to the True Vec- tor Field $\mathbf{v}^{\star}$}

In this section, we combine the continuity of the inverse map (Corollary 4.10) and the convergence of the posterior measure to $\theta\left(\mathbf{v}^{\star}\right)$ (Lemma 4.21) to show that, as the number of observations goes to infinity, the posterior converges weakly to a Dirac measure centered at $\mathbf{V}^{\star}$

Theorem 4.22 (Convergence of Posterior to a Dirac). Let $\left\{\left(t_{j}, \mathbf{x}_{j}\right)\right\}_{j=1}^{\infty}$ be a sequence of observation points uniformly distributed in $[0, T] \times \mathbb{T}^{2}$ and denote the associated data by $\left\{\mathcal{Y}_{j}\right\}_{j=1}^{\infty}$, where

$$
\mathcal{Y}_{j}=\mathcal{G}_{j}\left(\mathbf{v}^{\star}\right)+\eta_{j}
$$

for i.i.d. observational noise $\eta_{j} \sim N\left(0, \sigma_{\eta}^{2}\right)$, some true vector field $\mathbf{v}^{\star}$, and parameter-toobservable (forward) map $\mathcal{G}_{j}$ given by Definition 4.5 .

Furthermore, assume that the true background flow $\mathbf{v}^{\star}$ satisfies Assumption 4.3 and the prior measure $\mu_{0}$ satisfies Assumption 4.17 and Assumption 4.18.

Let $\mu_{\mathcal{Y}}^{N}$ be the Bayesian posterior measure on $H$ for $N$ observations, given by

$$
\mu_{\mathcal{Y}}^{N}(d \mathbf{v})=\frac{1}{Z} \exp \left[-\frac{1}{2 \sigma_{\eta}^{2}} \sum_{j=1}^{N}\left(\mathcal{Y}_{j}-\mathcal{G}_{j}(\mathbf{v})\right)^{2}\right] \mu_{0}(d \mathbf{v})
$$

where $Z$ is the normalization

$$
Z=\int_{H} \exp \left[-\frac{1}{2 \sigma_{\eta}^{2}} \sum_{j=1}^{N}\left(\mathcal{Y}_{j}-\mathcal{G}_{j}(\mathbf{v})\right)^{2}\right] \mu_{0}(d \mathbf{v})
$$

Then $\mu_{\mathcal{Y}}^{N} \rightarrow \delta_{\mathbf{v}^{\star}}$ a.s. 
Proof. Let $\epsilon>0$. Let $A$ be an open subset of $H$. To show weak convergence, we need to show

$$
\liminf _{N \rightarrow \infty} \mu_{\mathcal{Y}}^{N}(A) \geq \delta_{\mathbf{v}^{\star}}(A) .
$$

If $\mathbf{v}^{\star} \notin A$, then $\delta_{\mathbf{v}^{\star}}(A)=0$ so the result is trivial.

Now consider $\mathbf{v}^{\star} \in A$. As guaranteed Lemma 4.20, we choose $R>0$ so that

$$
\mu_{\mathcal{Y}}^{N}\left(\left(B_{V}^{R}\left(\mathbf{v}^{\star}\right)\right)^{c}\right)<\frac{\epsilon}{2}
$$

Since $A$ is open there exists an $\gamma>0$ such that $B_{H}^{\gamma}\left(\mathbf{v}^{\star}\right) \subset A$. Then by Corollary 4.11 there exists a $\delta>0$ such that $H_{\delta} \cap B_{V}^{R}\left(\mathbf{v}^{\star}\right) \subset B_{H}^{\gamma}\left(\mathbf{v}^{\star}\right) \subset A$. Then

$$
\mu_{\mathcal{Y}}^{N}(A) \geq \mu_{\mathcal{Y}}^{N}\left(B_{H}^{\gamma}\left(\mathbf{v}^{\star}\right)\right) \geq \mu_{\mathcal{Y}}^{N}\left(H_{\delta} \cap B_{V}^{R}\left(\mathbf{v}^{\star}\right)\right) \geq \mu_{\mathcal{Y}}^{N}\left(H_{\delta}\right)-\mu_{\mathcal{Y}}^{N}\left(\left(B_{V}^{R}\left(\mathbf{v}^{\star}\right)\right)^{c}\right) \geq \mu_{\mathcal{Y}}^{N}\left(H_{\delta}\right)-\frac{\epsilon}{2} .
$$

However, Lemma 4.21 states that there exists an $N^{\star}$ such that for all $N>N^{\star}$,

$$
\mu_{\mathcal{Y}}^{N}\left(H_{\delta}\right)>1-\frac{\epsilon}{2} \quad \text { a.s. }
$$

Therefore for all $N>N^{\star}$,

$$
\mu_{\mathcal{Y}}^{N}(A) \geq \mu_{\mathcal{Y}}^{N}\left(H_{\delta}\right)-\frac{\epsilon}{2}>1-\epsilon=\delta_{\mathbf{v}^{\star}}(A)-\epsilon \quad \text { a.s. }
$$

Thus, $\liminf \operatorname{in}_{N \rightarrow \infty} \mu_{\mathcal{Y}}^{N}(A) \geq \delta_{\mathbf{v}^{\star}}(A)$ with probability 1 .

In the above section, we have shown conditions under which the posterior measure converges to a Dirac measure concentrated at the true background flow $\mathbf{v}^{\star}$ - that is, the effects of the 
observational error and the prior measure both are eliminated - as the number of observations grows large. The key requirements were that the support of the prior measure contain compact balls around $\mathbf{v}^{\star}$ (Assumption 4.17), that the prior not place too great a weight on background flows of low regularity (Assumption 4.18), that observations be taken for two different initial conditions (Assumption 4.4), and that the observation noise be i.i.d. Gaussian.

In the next chapter, we turn our attention to computing observables from the posterior $\mu_{\mathcal{Y}}$. 


\section{Chapter 5}

\section{Overview of MCMC Methods}

Markov Chain Monte Carlo (MCMC) methods are used to generate random samples from a target distribution with measure $\mu$. For Bayesian problems, $\mu$ will be the posterior measure. The methods do this by creating a Markov chain with a probability transition kernel $\mathcal{P}$ for which $\mu$ is an invariant measure. This chapter will describe key concepts in Markov chains, derive the Metropolis-Hastings algorithm - an important class of MCMC methods in infinite dimensions, and describe a few examples of these algorithms.

We start with the definition of a Markov kernel:

Definition 5.1 (Markov Kernel). Let $(H, \mathcal{B})$ be a measurable space. A Markov kernel $\mathcal{P}:(H \times \mathcal{B}) \rightarrow[0,1]$ is a map such that

1. For any $B \in \mathcal{B}, \mathbf{v} \mapsto \mathcal{P}(\mathbf{v}, B)$ is a measurable function

2. For any $\mathbf{v} \in H, B \mapsto P(\mathbf{v}, B)$ is a probability measure

The Markov kernel describes roughly the probability that the chain migrates from point $\mathbf{v}$ to somewhere in the set $B$. Then we have the following two definitions that are essential to the theory of MCMC methods:

Definition 5.2 (Invariant Measure). Let $(H, \mathcal{B})$ be a measurable space. The probability 
measure $\mu$ is an invariant measure of Markov kernel $\mathcal{P}$ if

$$
\mu \mathcal{P}=\mu
$$

i.e. for any $B \in \mathcal{B}$,

$$
\mu(B)=\int_{H} \mathcal{P}(\mathbf{v}, B) \mu(d \mathbf{v}) .
$$

Definition 5.3 (Detailed Balance, Reversible). A Markov kernel $\mathcal{P}$ satisfies detailed balance with respect to probability measure $\mu$ if

$$
\mu(d \mathbf{v}) \mathcal{P}(\mathbf{v}, d \tilde{\mathbf{v}})=\mu(d \tilde{\mathbf{v}}) \mathcal{P}(\tilde{\mathbf{v}}, d \mathbf{v})
$$

for any $\mathbf{v}, \tilde{\mathbf{v}} \in H$. The associated Markov chain is then said to be reversible with respect to $\mu$.

Note that by integrating across $\tilde{\mathbf{v}}$ in (5.3) and using Fubini's theorem, we see that a Markov kernel satisfying detailed balance with respect to $\mu$ also has $\mu$ as an invariant measure.

\subsection{Metropolis-Hastings in Infinite Dimensions}

While known methods exist for generating pseudo-random samples from certain well-understood distributions, such as the uniform distribution or normal distribution, in many cases the goal of MCMC methods is to sample from distributions that may be much more complicated, or may only be understood in relation to other distributions. The Metropolis-Hastings algorithm ([41],[60]), which is the basis of many of the most commonly-used MCMC algorithms, provides a two-step framework for sampling from complicated measures:

1. Sample from a well-understood distribution (e.g., a Gaussian). This sample is called 
the proposal.

2. Accept the proposal with probability $\alpha$, a function of the previous sample and the proposal. If the proposal is rejected, set the next sample to be equal to the current sample.

As long as $\alpha$ is chosen correctly, the resulting Markov chain will have $\mu$ as an invariant measure.

Here we derive the Metropolis-Hastings kernel (i.e., the acceptance ratio $\alpha$ for given proposal kernel) in infinite dimensions. The derivation in this case is more technical than in $\mathbb{R}^{n}$ since Lebesgue measure is not defined in infinite dimensions. For a derivation in finite dimensions, we refer the reader to [46]. The extension of the derivation from finite dimensions to general state spaces originates from [82]. A detailed discussion of infinite-dimensional sampling is provided in Section 5 of [24]. A discussion of several of the algorithms mentioned below and their application to infinite-dimensional problems is provided in [12].

Definition 5.4 (Metropolis-Hastings Kernel). Suppose $\mathcal{Q}$ is a Markov kernel on a Polish space $H$ and $\mu$ is a Borel probability measure on $H$. Then the Metropolis-Hastings kernel $\mathcal{P}$ for proposal kernel $\mathcal{Q}$ and acceptance probability $\alpha$ is

$$
\mathcal{P}(\mathbf{v}, d \tilde{\mathbf{v}})=\alpha(\mathbf{v}, \tilde{\mathbf{v}}) \mathcal{Q}(\mathbf{v}, d \tilde{\mathbf{v}})+\delta_{\mathbf{v}}(d \tilde{\mathbf{v}}) \int_{H}(1-\alpha(\mathbf{v}, \mathbf{w})) \mathcal{Q}(\mathbf{v}, d \mathbf{w})
$$

where $\mathbf{v}, \tilde{\mathbf{v}} \in H$ and $\delta_{\mathbf{v}}$ is the Dirac measure concentrated at $\mathbf{v}$.

The first term represents acceptance of the proposal (with probability $\alpha(\mathbf{v}, \tilde{\mathbf{v}})$ ). The second term sums across all possible rejections, where $\mathbf{w} \in H$ is proposed and rejected with probability $1-\alpha(\mathbf{v}, \mathbf{w})$, so that $\mathbf{v}$ is retained.

Algorithmically, the Metropolis-Hastings kernel Definition 5.4 is given by Algorithm 1 . 


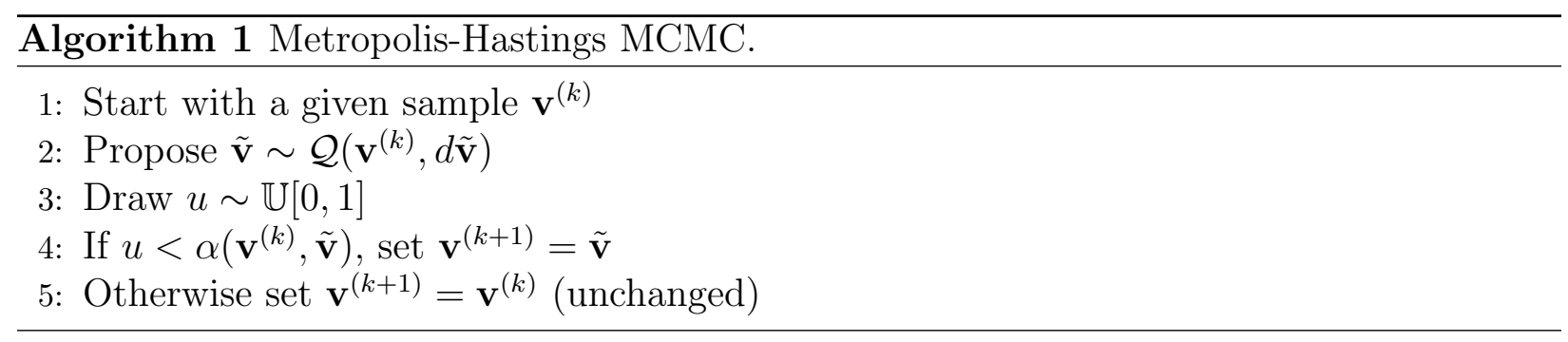

Note that Definition 5.4 and Algorithm 1 are equivalent, since for arbitrary $g \in C_{b}(H)$

$$
\begin{aligned}
\mathbb{E}_{\tilde{\mathbf{v}}} g & \left(\tilde{\mathbf{v}} \mathbb{1}_{u<\alpha(\mathbf{v}, \tilde{\mathbf{v}})}+\mathbf{v} \mathbb{1}_{u \geq \alpha(\mathbf{v}, \tilde{\mathbf{v}})}\right) \\
& =\mathbb{E}_{\mathbf{w}} \mathbb{E}_{\tilde{\mathbf{v}}}\left[g\left(\tilde{\mathbf{v}} \mathbb{1}_{u<\alpha(\mathbf{v}, \tilde{\mathbf{v}})}+\mathbf{v} \mathbb{1}_{u \geq \alpha(\mathbf{v}, \tilde{\mathbf{v}})} \mid \mathbf{w}\right)\right] \\
& =\mathbb{E}_{\mathbf{w}} g(\mathbf{w}) \alpha(\mathbf{v}, \mathbf{w})+g(\mathbf{v})(1-\alpha(\mathbf{v}, \mathbf{w})) \\
& =\int g(\mathbf{w}) \alpha(\mathbf{v}, \mathbf{w}) \mathcal{Q}(\mathbf{v}, d \mathbf{w})+g(\mathbf{v}) \int(1-\alpha(\mathbf{v}, \mathbf{w})) \mathcal{Q}(\mathbf{v}, d \mathbf{w}) \\
& =\int g(\tilde{\mathbf{v}}) \alpha(\mathbf{v}, \tilde{\mathbf{v}}) \mathcal{Q}(\mathbf{v}, d \tilde{\mathbf{v}})+\int g(\tilde{\mathbf{v}}) \delta_{\mathbf{v}}(d \tilde{\mathbf{v}}) \int(1-\alpha(\mathbf{v}, \mathbf{w})) \mathcal{Q}(\mathbf{v}, d \mathbf{w}) \\
& =\int g(\tilde{\mathbf{v}}) \mathcal{P}(\mathbf{v}, d \tilde{\mathbf{v}}) .
\end{aligned}
$$

We now show how the acceptance probability $\alpha$ can be chosen so that the the target measure is invariant with respect to the Metropolis-Hastings kernel. This derivation is taken largely from [24].

Theorem 5.5 (Metropolis-Hastings Acceptance Probability). Let $\mathcal{P}$ be the Metropolis-Hastings kernel for a proposal kernel $\mathcal{Q}$ and acceptance probability $\alpha$, given by (5.4). Let $\nu, \nu^{\perp}$ be the product measures

$$
\begin{aligned}
\nu(d \mathbf{v}, d \tilde{\mathbf{v}}) & =\mu(d \mathbf{v}) \mathcal{Q}(\mathbf{v}, d \tilde{\mathbf{v}}) \\
\nu^{\perp}(d \mathbf{v}, d \tilde{\mathbf{v}}) & =\mu(d \tilde{\mathbf{v}}) \mathcal{Q}(\tilde{\mathbf{v}}, d \mathbf{v})
\end{aligned}
$$

and assume that $\nu, \nu^{\perp}$ are mutually absolutely continuous. 
Then $\mathcal{P}$ will have $\mu$ as an invariant measure if

$$
\alpha(\mathbf{v}, \tilde{\mathbf{v}})=1 \wedge \frac{d \nu^{\perp}}{d \nu}(\mathbf{v}, \tilde{\mathbf{v}})
$$

Proof. Note that by definition

$$
\nu^{\perp}(d \mathbf{v}, d \tilde{\mathbf{v}})=\nu(d \tilde{\mathbf{v}}, d \mathbf{v})
$$

Also, since

$$
\begin{aligned}
\nu(d \mathbf{v}, d \tilde{\mathbf{v}}) & =\nu^{\perp}(d \tilde{\mathbf{v}}, d \mathbf{v})=\frac{d \nu^{\perp}}{d \nu}(\tilde{\mathbf{v}}, \mathbf{v}) \nu(d \tilde{\mathbf{v}}, d \mathbf{v})=\frac{d \nu^{\perp}}{d \nu}(\tilde{\mathbf{v}}, \mathbf{v}) \nu^{\perp}(d \mathbf{v}, d \tilde{\mathbf{v}}) \\
& =\frac{d \nu^{\perp}}{d \nu}(\tilde{\mathbf{v}}, \mathbf{v}) \frac{d \nu^{\perp}}{d \nu}(\mathbf{v}, \tilde{\mathbf{v}}) \nu(d \mathbf{v}, d \tilde{\mathbf{v}})
\end{aligned}
$$

we have

$$
\frac{d \nu^{\perp}}{d \nu}(\tilde{\mathbf{v}}, \mathbf{v}) \frac{d \nu^{\perp}}{d \nu}(\mathbf{v}, \tilde{\mathbf{v}})=1
$$

Let $\phi \in C_{b}(H \times H)$ be an arbitrary test function. Let $A=\left\{(\mathbf{v}, \tilde{\mathbf{v}}): \frac{d \nu^{\perp}}{d \nu}(\mathbf{v}, \tilde{\mathbf{v}})<1\right\}$. Note that for $(\mathbf{v}, \tilde{\mathbf{v}}) \in A^{c}, \frac{d \nu^{\perp}}{d \nu}(\tilde{\mathbf{v}}, \mathbf{v}) \leq 1$ by (5.9). Then, applying (5.7) and the definition (5.6) of 
$\alpha$ yields

$$
\begin{aligned}
\int_{H} \int_{H} \phi(\mathbf{v}, \tilde{\mathbf{v}}) & \mu(d \mathbf{v}) \alpha(\mathbf{v}, \tilde{\mathbf{v}}) \mathcal{Q}(\mathbf{v}, d \tilde{\mathbf{v}}) \\
& =\int_{H} \int_{H} \phi(\mathbf{v}, \tilde{\mathbf{v}}) \nu(d \mathbf{v}, d \tilde{\mathbf{v}})\left[\frac{d \nu^{\perp}}{d \nu}(\mathbf{v}, \tilde{\mathbf{v}}) \mathbb{1}_{A}(\mathbf{v}, \tilde{\mathbf{v}})+\mathbb{1}_{A^{c}}(\mathbf{v}, \tilde{\mathbf{v}})\right] \\
& =\int_{H} \int_{H} \phi(\mathbf{v}, \tilde{\mathbf{v}})\left[\nu^{\perp}(d \mathbf{v}, d \tilde{\mathbf{v}}) \mathbb{1}_{A}(\mathbf{v}, \tilde{\mathbf{v}})+\nu(d \mathbf{v}, d \tilde{\mathbf{v}}) \mathbb{1}_{A^{c}}(\mathbf{v}, \tilde{\mathbf{v}})\right] \\
& =\int_{H} \int_{H} \phi(\mathbf{v}, \tilde{\mathbf{v}})\left[\nu(d \tilde{\mathbf{v}}, d \mathbf{v}) \mathbb{1}_{A}(\mathbf{v}, \tilde{\mathbf{v}})+\nu^{\perp}(d \tilde{\mathbf{v}}, d \mathbf{v}) \mathbb{1}_{A^{c}}(\mathbf{v}, \tilde{\mathbf{v}})\right] \\
& =\int_{H} \int_{H} \phi(\mathbf{v}, \tilde{\mathbf{v}})\left[\nu(d \tilde{\mathbf{v}}, d \mathbf{v}) \mathbb{1}_{A}(\mathbf{v}, \tilde{\mathbf{v}})+\frac{d \nu^{\perp}}{d \nu}(\tilde{\mathbf{v}}, \mathbf{v}) \nu(d \tilde{\mathbf{v}}, d \mathbf{v}) \mathbb{1}_{A^{c}}(\mathbf{v}, \tilde{\mathbf{v}})\right] \\
= & \int_{H} \int_{H} \phi(\mathbf{v}, \tilde{\mathbf{v}}) \nu(d \tilde{\mathbf{v}}, d \mathbf{v}) \alpha(\tilde{\mathbf{v}}, \mathbf{v}) .
\end{aligned}
$$

Also

$$
\begin{aligned}
\int_{H} \int_{H} \phi(\mathbf{v}, \tilde{\mathbf{v}}) \mu(d \mathbf{v}) \delta_{\mathbf{v}}(d \tilde{\mathbf{v}}) & \int_{H}(1-\alpha(\mathbf{v}, \mathbf{w})) \mathcal{Q}(\mathbf{v}, d \mathbf{w}) \\
& =\int_{H} \phi(\mathbf{v}, \mathbf{v}) \mu(d \mathbf{v}) \int_{H}(1-\alpha(\mathbf{v}, \mathbf{w})) \mathcal{Q}(\mathbf{v}, d \mathbf{w}) \\
& =\int_{H} \phi(\tilde{\mathbf{v}}, \tilde{\mathbf{v}}) \mu(d \tilde{\mathbf{v}}) \int_{H}(1-\alpha(\tilde{\mathbf{v}}, \mathbf{w})) \mathcal{Q}(\tilde{\mathbf{v}}, d \mathbf{w}) \\
& =\int_{H} \int_{H} \phi(\mathbf{v}, \tilde{\mathbf{v}}) \mu(d \tilde{\mathbf{v}}) \delta_{\tilde{\mathbf{v}}}(d \mathbf{v}) \int_{H}(1-\alpha(\tilde{\mathbf{v}}, \mathbf{w})) \mathcal{Q}(\tilde{\mathbf{v}}, d \mathbf{w}) .
\end{aligned}
$$


Assembling the last two results and using the definition (5.4) of $\mathcal{P}$ yields

$$
\begin{aligned}
\int_{H} \int_{H} \phi(\mathbf{v}, \tilde{\mathbf{v}}) \mu & (d \mathbf{v}) \mathcal{P}(\mathbf{v}, d \tilde{\mathbf{v}}) \\
= & \int_{H} \int_{H} \phi(\mathbf{v}, \tilde{\mathbf{v}}) \mu(d \mathbf{v})\left[\alpha(\mathbf{v}, \tilde{\mathbf{v}}) \mathcal{Q}(\mathbf{v}, d \tilde{\mathbf{v}})+\delta_{\mathbf{v}}(d \tilde{\mathbf{v}}) \int_{H}(1-\alpha(\mathbf{v}, \mathbf{w})) \mathcal{Q}(\mathbf{v}, d \mathbf{w})\right] \\
= & \int_{H} \int_{H} \phi(\mathbf{v}, \tilde{\mathbf{v}}) \nu(d \tilde{\mathbf{v}}, d \mathbf{v}) \alpha(\tilde{\mathbf{v}}, \mathbf{v}) \\
& +\int_{H} \int_{H} \phi(\mathbf{v}, \tilde{\mathbf{v}}) \mu(d \tilde{\mathbf{v}}) \delta_{\tilde{\mathbf{v}}}(d \mathbf{v}) \int_{H}(1-\alpha(\tilde{\mathbf{v}}, \mathbf{w})) \mathcal{Q}(\tilde{\mathbf{v}}, d \mathbf{w}) \\
= & \int_{H} \int_{H} \phi(\mathbf{v}, \tilde{\mathbf{v}}) \mu(d \tilde{\mathbf{v}})\left[\mathcal{P}(\tilde{\mathbf{v}}, d \mathbf{v}) \alpha(\tilde{\mathbf{v}}, \mathbf{v})+\delta_{\tilde{\mathbf{v}}}(d \mathbf{v}) \int_{H}(1-\alpha(\tilde{\mathbf{v}}, \mathbf{w})) \mathcal{Q}(\tilde{\mathbf{v}}, d \mathbf{w})\right] \\
= & \int_{H} \int_{H} \phi(\mathbf{v}, \tilde{\mathbf{v}}) \mu(d \tilde{\mathbf{v}}) \mathcal{P}(\tilde{\mathbf{v}}, d \mathbf{v}) .
\end{aligned}
$$

Therefore $\mu(d \mathbf{v}) \mathcal{P}(\mathbf{v}, d \tilde{\mathbf{v}})=\mu(d \tilde{\mathbf{v}}) \mathcal{P}(\tilde{\mathbf{v}}, d \mathbf{v})$, so $\mathcal{P}$ is reversible with respect to $\mu$. Therefore $\mu$ is an invariant measure for $\mathcal{P}$.

The following corollary addresses the case where we do not know the target measure $\mu$ directly, but rather, as in the Bayesian case, know it in terms of a density with respect to the prior $\mu_{0}$.

Corollary 5.6 (Acceptance Probability in terms of $\left.\mu_{0}\right)$. Let $\mathcal{P}$ be the Metropolis-Hastings kernel for a proposal kernel $\mathcal{Q}$ and acceptance probability $\alpha$, given by (5.4). Suppose that a measure $\mu$ is given by its density with respect to another measure $\mu_{0}$ :

$$
\mu(d \mathbf{v})=\frac{1}{Z} \exp [-\Phi(\mathbf{v})] \mu_{0}(d \mathbf{v})
$$

Then $\mathcal{P}$ will have $\mu$ as an invariant measure if

$$
\alpha(\mathbf{v}, \tilde{\mathbf{v}})=1 \wedge \exp [\Phi(\mathbf{v})-\Phi(\tilde{\mathbf{v}})] \frac{d \nu_{0}^{\perp}}{d \nu_{0}}(\mathbf{v}, \tilde{\mathbf{v}})
$$


where $\nu_{0}, \nu_{0}^{\perp}$ are the product measures

$$
\begin{aligned}
\nu_{0}(d \mathbf{v}, d \tilde{\mathbf{v}}) & =\mu_{0}(d \mathbf{v}) \mathcal{Q}(\mathbf{v}, d \tilde{\mathbf{v}}) \\
\nu_{0}^{\perp}(d \mathbf{v}, d \tilde{\mathbf{v}}) & =\mu_{0}(d \tilde{\mathbf{v}}) \mathcal{Q}(\tilde{\mathbf{v}}, d \mathbf{v}) .
\end{aligned}
$$

Proof. The product measures $\nu$ and $\nu^{\perp}$ from (5.5) can be written as

$$
\begin{aligned}
\nu(d \mathbf{v}, d \tilde{\mathbf{v}}) & =\mu(d \mathbf{v}) \mathcal{Q}(\mathbf{v}, d \tilde{\mathbf{v}})=\frac{1}{Z} \exp [-\Phi(\mathbf{v})] \mu_{0}(d \mathbf{v}) \mathcal{Q}(\mathbf{v}, d \tilde{\mathbf{v}})=\frac{1}{Z} \exp [-\Phi(\mathbf{v})] \nu_{0}(d \mathbf{v}, d \tilde{\mathbf{v}}) \\
\nu^{\perp}(d \mathbf{v}, d \tilde{\mathbf{v}})=\mu(d \tilde{\mathbf{v}}) \mathcal{Q}(\tilde{\mathbf{v}}, d \mathbf{v}) & =\frac{1}{Z} \exp [-\Phi(\tilde{\mathbf{v}})] \mu_{0}(d \tilde{\mathbf{v}}) \mathcal{Q}(\tilde{\mathbf{v}}, d \mathbf{v})=\frac{1}{Z} \exp [-\Phi(\tilde{\mathbf{v}})] \nu_{0}^{\perp}(d \mathbf{v}, d \tilde{\mathbf{v}})
\end{aligned}
$$

Then by Theorem 5.5 the acceptance ratio

$$
\alpha(\mathbf{v}, \tilde{\mathbf{v}})=1 \wedge \frac{d \nu^{\perp}}{d \nu}(\mathbf{v}, \tilde{\mathbf{v}})=1 \wedge \exp [\Phi(\mathbf{v})-\Phi(\tilde{\mathbf{v}})] \frac{d \nu_{0}^{\perp}}{d \nu_{0}}(\mathbf{v}, \tilde{\mathbf{v}})
$$

ensures that $\mu$ is an invariant measure for $\mathcal{P}$.

In the methods that follow below, the proposal kernel $\mathcal{Q}$ is often reversible with respect to the prior, i.e.

$$
\mu_{0}(d \mathbf{v}) \mathcal{Q}(\mathbf{v}, d \tilde{\mathbf{v}})=\mu_{0}(d \tilde{\mathbf{v}}) \mathcal{Q}(\tilde{\mathbf{v}}, d \mathbf{v})
$$

In this case, $\frac{d \nu_{0}^{\perp}}{d \nu_{0}}=1$ and the acceptance ratio (5.11) reduces to simply

$$
\alpha(\mathbf{v}, \tilde{\mathbf{v}})=1 \wedge \exp [\Phi(\mathbf{v})-\Phi(\tilde{\mathbf{v}})]
$$




\subsection{The Independence Sampler}

If we choose $\mathcal{Q}(\mathbf{v}, d \tilde{\mathbf{v}})$ independent of $\mathbf{v}$, then each proposal is drawn at random from the same distribution, independent of the previous sample. This is the independence sampler. In particular, if we choose $\mathcal{Q}(\mathbf{v}, d \tilde{\mathbf{v}})=\mu_{0}(d \tilde{\mathbf{v}})$ so that $\tilde{\mathbf{v}} \sim \mu_{0}$, then prior reversibility $(5.13)$ is satisfied since

$$
\nu_{0}(d \mathbf{v}, d \tilde{\mathbf{v}})=\mu_{0}(d \mathbf{v}) \mu_{0}(d \tilde{\mathbf{v}})=\nu_{0}^{\perp}(d \mathbf{v}, d \tilde{\mathbf{v}})
$$

Then the acceptance ratio for the independence sampler is given by (5.14).

Algorithm 2 describes the resulting Independence Sampler (see also Algorithm 5.9 of [24]).

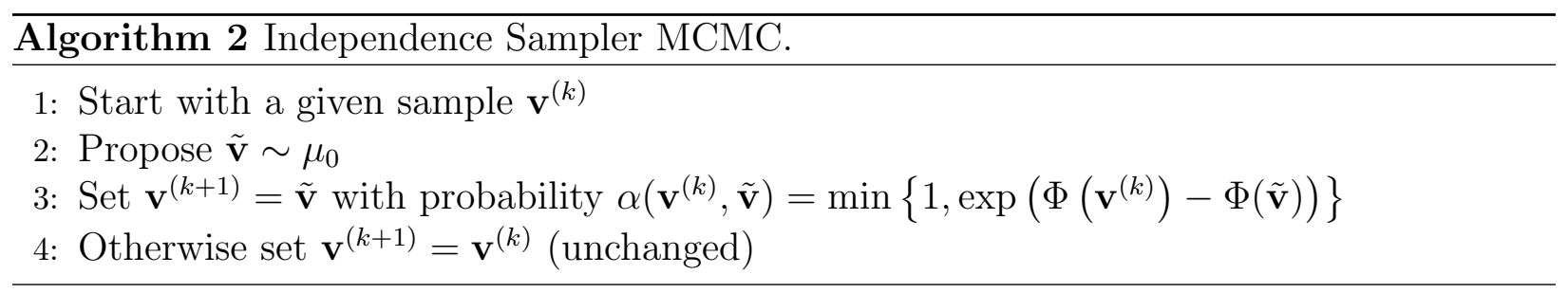

\subsection{Preconditioned Crank-Nicolson (pCN) MCMC}

The preconditioned Crank-Nicolson ( $\mathrm{pCN}$ ) method was introduced in [21] and provides a method for efficient sampling from mean-zero Gaussian measures. Consider a Gaussian measure on Hilbert space $H$ with covariance $\mathcal{C}$.[23] Then the stochastic evolution equation

$$
d \mathbf{v}_{t}=-\mathbf{v} d t+\sqrt{2 \mathcal{C}} d W_{t}
$$

defines an Ornstein-Uhlenbeck process with invariant measure $N(0, \mathcal{C})$. This equation can be discretized to yield samples that are distributed (approximately) like $N(0, \mathcal{C})$. One such 
discretization is the preconditioned Crank-Nicolson (pCN):

$$
\mathbf{v}_{j+1}-\mathbf{v}_{j}=-\frac{1}{2}\left(\mathbf{v}_{j+1}+\mathbf{v}_{j}\right) \Delta t+\sqrt{2 \Delta t} \xi_{j}
$$

where $\xi_{j} \sim N(0, \mathcal{C})$. Defining $\beta=\frac{2 \sqrt{2 \Delta t}}{2+\Delta t}$ and solving for $\mathbf{v}_{j+1}$ yields

$$
\mathbf{v}_{j+1}=\sqrt{1-\beta^{2}} \mathbf{v}_{j}+\beta \xi_{j} .
$$

This discretization can be used as a Metropolis-Hastings proposal. For Gaussian prior $N(0, \mathcal{C})$, the proposal kernel is reversible with respect to the prior, so the acceptance ratio is given by (5.14).

The proposal (5.17) and acceptance ratio form an MCMC algorithm for Gaussian priors $\mu_{0}=N(0, \mathcal{C})$, which is summarized in Algorithm 3.

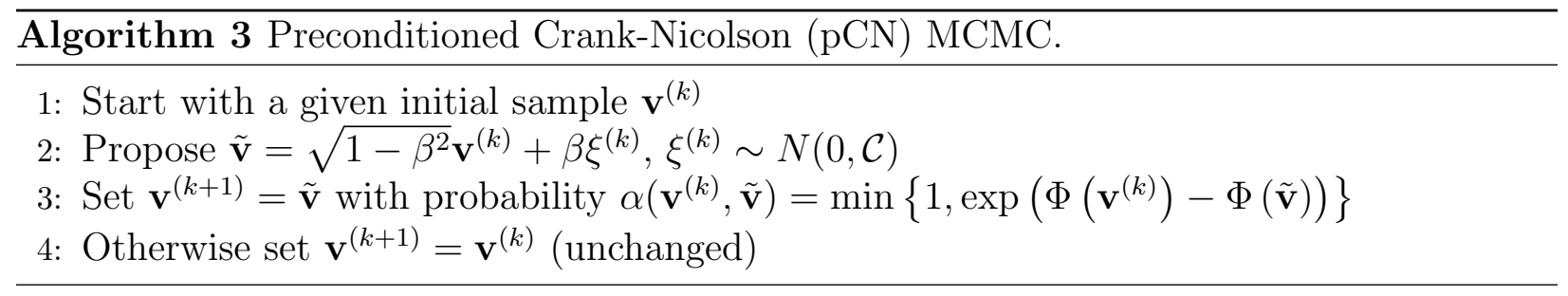

The method can be extended to uncentered Gaussian priors by shifting the mean of the OU process (5.15). However, it does not apply to non-Gaussian priors.

The free parameter $\beta \in(0,1]$ can be tuned to the problem. Smaller values of $\beta$ keep proposals close to the current sample. Larger values of $\beta$ bias proposals toward the origin (i.e., toward the center of the prior - in fact, $\beta=1$ is the independence sampler) and can produce larger jumps between samples but also potentially comes with the costs associated with smaller acceptance rates. See, e.g., Section 5.2.5 of [21] or Section 8.3.2 for more detailed discussion. 


\subsection{Metropolis-Adjusted Langevin (MALA) MCMC}

The Metropolis-adjusted Langevin (MALA) MCMC method, which leverages Langevin diffusions to provided data-informed proposals, was first investigated in the mid-1990s.[37, 72] Like the pCN method, the MALA method was extended to infinite dimensions in [21] and provides a method for efficient sampling from posteriors for mean-zero Gaussian priors. Unlike $\mathrm{pCN}$, in which proposals are sampled from the prior, MALA incorporates the data in the form of the Fréchet derivative of the potential $\Phi$. In fact, MALA's proposals are sampled directly from the posterior, up to discretization error.

As in Section 5.3, consider a Gaussian measure on Hilbert space $H$ with covariance $\mathcal{C}$. [23] Then the following stochastic evolution equation

$$
d \mathbf{v}_{t}=-(\mathbf{v}+\mathcal{C} D \Phi(\mathbf{v})) d t+\sqrt{2 \mathcal{C}} d W_{t}
$$

defines a Langevin diffusion that has as its invariant measure the Bayesian posterior $\mu$

given by $(3.25)$ with $\mu_{0}=N(0, \mathcal{C})$. Then discretizing this SDE should yield samples that are distributed like $\mu$, up to discretization error. One such discretization is semi-implicit scheme:

$$
\begin{aligned}
\mathbf{v}_{j+1}-\mathbf{v}_{j} & =-\frac{1}{2}\left(\mathbf{v}_{j+1}+\mathbf{v}_{j}+\mathcal{C} D \Phi\left(\mathbf{v}_{j+1}\right)+\mathcal{C} D \Phi\left(\mathbf{v}_{j}\right)\right) h+\sqrt{2 \mathcal{C} h} \xi_{j} \\
& \approx-\frac{1}{2}\left(\mathbf{v}_{j+1}+\mathbf{v}_{j}\right) h+\mathcal{C} D \Phi\left(\mathbf{v}_{j}\right) h+\sqrt{2 h} \xi_{j}
\end{aligned}
$$

where $\xi_{j} \sim N(0, \mathcal{C})$. Solving for $\mathbf{v}_{j+1}$ yields

$$
\mathbf{v}_{j+1}=\frac{2-h}{2+h} \mathbf{v}_{j}-\frac{2 h}{2+h} \mathcal{C} D \Phi\left(\mathbf{v}_{j}\right)+\frac{\sqrt{8 h}}{2+h} \xi_{j} .
$$


The acceptance ratio, given in [21], is

$$
\alpha(\mathbf{v}, \tilde{\mathbf{v}})=1 \wedge \exp (\rho(\mathbf{v}, \tilde{\mathbf{v}})-\rho(\tilde{\mathbf{v}}, \mathbf{v}))
$$

where

$$
\rho(\mathbf{v}, \tilde{\mathbf{v}})=\Phi(\mathbf{v})+\frac{1}{2}\langle\tilde{\mathbf{v}}-\mathbf{v}, D \Phi(\mathbf{v})\rangle+\frac{h}{4}\langle\mathbf{v}+\tilde{\mathbf{v}}, D \Phi(\mathbf{v})\rangle+\frac{h}{4}\left\|\mathcal{C}^{\frac{1}{2}} D \Phi(\mathbf{v})\right\|^{2} .
$$

The resulting MALA MCMC algorithm is outlined in Algorithm 4. Note that the algorithm allows the practitioner a choice of the step size $h$. As $h \rightarrow 0$, it can be show that proposals will be distributed like the posterior $\mu$ and the acceptance probability converges to 1.[14] Larger $h$ can produce larger jumps, reducing the correlation between samples, but potentially also reducing the acceptance rate.

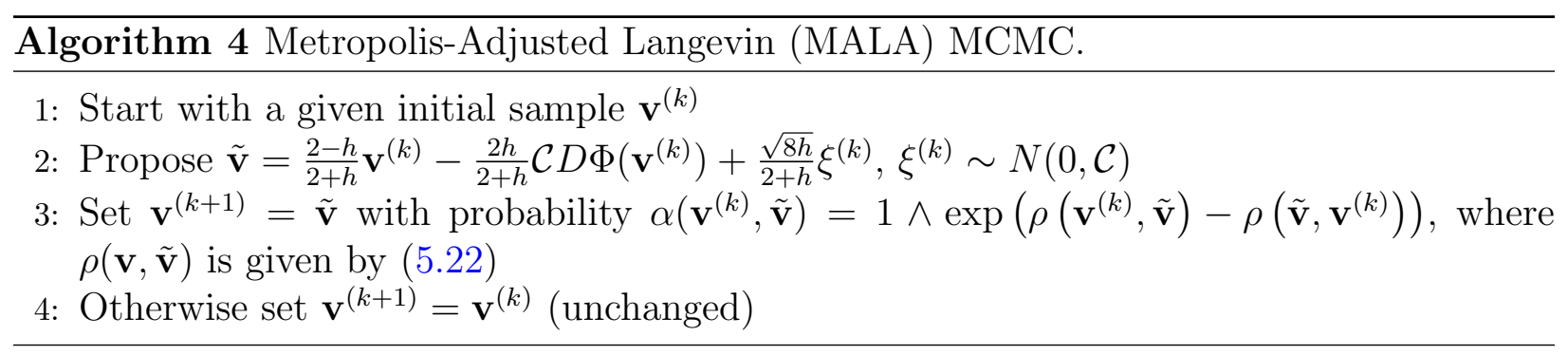

\subsection{Hamiltonian Monte Carlo (HMC)}

Hamiltonian, or Hybrid, Monte Carlo (HMC) uses Hamiltonian mechanics to generate MetropolisHastings proposals from the (approximate) posterior distribution. In the HMC method, the MCMC samples are treated as generalized position coordinates, which we will denote $\mathbf{q}$, in an appropriately chosen Hamiltonian dynamical system. The samples are then augmented by a generalized momentum $\mathbf{p}$ drawn at random. This can be thought of as a "particle" at 
position q "excited" with kinetic energy. The particle is then allowed to move in an energy landscape given by the prior $\mu_{0}$ and potential $\Phi$ according to Hamilton's laws of motion. The excitation allows the method to generate large jumps between samples, mitigating the "random walk" behavior seen in the pCN or MALA methods, while the use of Hamiltonian dynamics keeps the acceptance probability high. The method was introduced in [29]. An extensive description of both theoretical underpinnings and practical implementation is provided in [65]. It was extended to infinite dimensions in [11], with the restriction that the prior $\mu_{0}$ be Gaussian, and expanded upon in [12] to incorporate local preconditioning in the sampling.

\section{Hamiltonian Mechanics}

Hamiltonian mechanics describes the interaction of generalized position $\mathbf{q}$ and momentum p coordinates which conserve the Hamiltonian $\mathcal{H}(\mathbf{q}, \mathbf{p})$, which is typically the total energy of the system and equal to the sum of the potential energy $\mathcal{U}(\mathbf{q})$ and kinetic energy $\mathcal{K}(\mathbf{p})$ :

$$
\mathcal{H}(\mathbf{q}, \mathbf{p})=\mathcal{U}(\mathbf{q})+\mathcal{K}(\mathbf{p})
$$

The first equation relates the change in position to the velocity, while the second relates the change in momentum to the change in potential energy (the force).

Because the dynamics preserve $\mathcal{H}$, they also preserve probability under the Gibbs measure

$$
\frac{1}{Z} \exp [-\mathcal{H}(\mathbf{q}, \mathbf{p})]=\frac{1}{Z} \exp [-\mathcal{U}(\mathbf{q})-\mathcal{K}(\mathbf{p})]=\frac{1}{Z} \exp [-\mathcal{U}(\mathbf{q})] \exp [-\mathcal{K}(\mathbf{p})]
$$

Then by proper choice of $\mathcal{U}$ and $\mathcal{K}$, the dynamics can be chosen to preserve a desired target

measure. In practice, this typically means selecting the potential energy to be sum of the 
potential $\Phi$ and the negative log of the prior density:

$$
\mathcal{U}(\mathbf{q})=\Phi(\mathbf{q})-\log \mu_{0}(d \mathbf{q})
$$

The kinetic energy can in principle be chosen freely. However, in infinite dimensions, some restrictions and modifications of the above derivation are necessary.[11, 12] First, the current theory requires that the prior be Gaussian. Second, it is desirable to use the velocity $\mathbf{w}=\mathcal{C} \mathbf{p}$ rather than momentum $\mathbf{p}$ as the auxiliary variable, where $\mathcal{C}$ is the covariance on the prior. Note that we have used $\mathcal{C}^{-1}$ as, effectively, a "mass matrix"; this ensures that w will be small in the tail of the distribution, where the covariances on the prior are going to zero. This yields the Hamiltonian

$$
\mathcal{H}(\mathbf{q}, \mathbf{p})=\Phi(\mathbf{q})+\frac{1}{2}\left\langle\mathbf{q}, \mathcal{C}^{-1} \mathbf{q}\right\rangle+\frac{1}{2}\left\langle\mathbf{w}, \mathcal{C}^{-1} \mathbf{w}\right\rangle
$$

Figure 5.1 displays the position of a particle moving in an example two-dimensional Hamiltonian system. Note that the augmented velocity allows the particle to jump between potential energy wells.

\section{The Leapfrog Step}

Here we describe how to numerically integrate the Hamiltonian dynamics to preserve the energy. Consider the infinite-dimensional Hamiltonian (5.26).

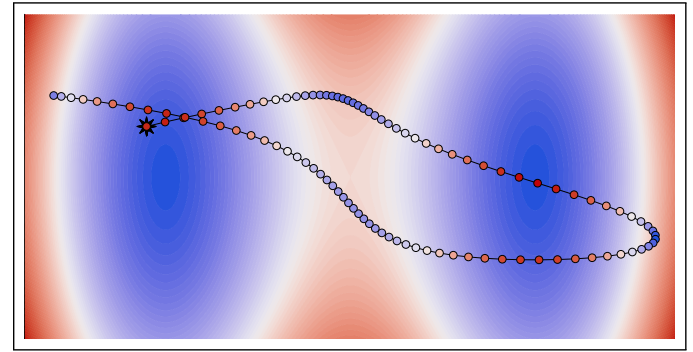

Figure 5.1: Particle moving in an example 2D Hamiltonian system (5.26).

Then the time evolution of the system is given by Hamilton's equations of motion:

$$
\begin{aligned}
\frac{d}{d t} \mathbf{q} & =\frac{\partial \mathcal{H}}{\partial \mathbf{p}}=\mathcal{C} \mathbf{p}=\mathbf{w} \\
\frac{d}{d t} \mathbf{w} & =\mathcal{C} \frac{d}{d t} \mathbf{p}=-\mathcal{C} \frac{\partial \mathcal{H}}{\partial \mathbf{q}}=-\mathbf{q}-\mathcal{C} D \Phi(\mathbf{q})
\end{aligned}
$$


In practice, these equations are typically integrated via the following system of equations

$$
\begin{aligned}
\mathbf{w}_{-} & =\mathbf{w}_{0}-\frac{\epsilon}{2} \mathcal{C} D \Phi\left(\mathbf{q}_{0}\right) \\
\mathbf{q}_{\epsilon} & =(\cos \epsilon) \mathbf{q}_{0}+(\sin \epsilon) \mathbf{w}_{-} \\
\mathbf{w}_{+} & =-(\sin \epsilon) \mathbf{q}_{0}+(\cos \epsilon) \mathbf{w}_{-} \\
\mathbf{w}_{\epsilon} & =\mathbf{w}_{+}-\frac{\epsilon}{2} \mathcal{C} D \Phi\left(\mathbf{q}_{\epsilon}\right) .
\end{aligned}
$$

This integration is reversible in time and therefore can be shown to better approximate the invariance of $\mathcal{H}$ (see [65]). The map given by (5.28) is called the "leapfrog" map.

\section{Acceptance Probability}

If the Hamiltonian differential equations could be solved analytically, the Hamiltonian would be preserved and therefore the method would be reversible with respect to the Gibbs measure (5.24). However, in general numerical integration must be used (as described above) and a Metropolis-Hastings accept-reject step is required to correct for any errors resulting from the time discretization. In particular, the acceptance ratio

$$
\alpha\left(\left(\mathbf{q}_{1}, \mathbf{p}_{1}\right),\left(\mathbf{q}_{2}, \mathbf{p}_{2}\right)\right)=1 \wedge \exp \left[\mathcal{H}\left(\mathbf{q}_{1}, \mathbf{p}_{1}\right)-\mathcal{H}\left(\mathbf{q}_{2}, \mathbf{p}_{2}\right)\right]=1 \wedge \exp [-\Delta \mathcal{H}]
$$

can be shown to maintain detailed balance [65].

However, in infinite dimensions the Hamiltonian is almost surely infinite, rendering the direct computation of $\Delta \mathcal{H}$ impossible. However, it is shown in [12] that the following definition is 
equivalent and well-defined:

$$
\begin{aligned}
\Delta \mathcal{H}= & \mathcal{H}\left(\mathbf{q}_{2}, \mathbf{p}_{2}\right)-\mathcal{H}\left(\mathbf{q}_{1}, \mathbf{p}_{1}\right) \\
= & \Phi\left(\mathbf{q}_{2}\right)-\Phi\left(\mathbf{q}_{1}\right)-\frac{\epsilon^{2}}{8}\left\{\left\|\mathcal{C}^{\frac{1}{2}} D \Phi\left(\mathbf{q}_{2}\right)\right\|^{2}-\left\|\mathcal{C}^{\frac{1}{2}} D \Phi\left(\mathbf{q}_{1}\right)\right\|^{2}\right\} \\
& -\frac{\epsilon}{2} \sum_{i=0}^{L-1}\left[\left\langle\mathbf{w}_{i}, D \Phi\left(\mathbf{q}_{i}\right)\right\rangle+\left\langle\mathbf{w}_{i+1}, D \Phi\left(\mathbf{q}_{i+1}\right)\right\rangle\right] .
\end{aligned}
$$

\section{Free Parameters}

The leapfrog integration scheme described above includes two parameters that can be chosen by the practitioner:

- $\epsilon$ is the integration step size. If we could integrate the dynamics perfectly, we would have $\Delta \mathcal{H}=0$, so the acceptance probability would be 1 . However, as $\epsilon$ increases, the integration will become less accurate and the computed Hamiltonian will deviate more from $\mathcal{H}\left(\mathbf{q}_{0}, \mathbf{w}_{0}\right)$, increasing $\Delta \mathcal{H}$ and reducing the acceptance ratio (5.29). At the same time, decreasing $\epsilon$ means more integration steps, and therefore more evaluations of $\Phi$ and $D \Phi$, per MCMC step, increasing computation time. Thus, based on our experience (see Section 8.3.2), the practitioner must pick an $\epsilon$ that is small enough to allow accurate integration, but not so small as to unnecessarily increase computational cost.

- $\tau$ is the integration end time. Longer integration time allows the Hamiltonian system to evolve more, so the final value of $\mathbf{q}$ may differ more from the initial condition. As such, larger $\tau$ will tend to make the MCMC samples less correlated with one another. However, the number of integration steps $L=\frac{\tau}{\epsilon}$ will increase linearly with $\tau$, increasing the number of evaluations of $\Phi$ and $D \Phi$ required, and thereby increasing the computational cost associated with each sample. 


\section{The Full Algorithm}

The full HMC algorithm combining the dynamics, numerical integration, and accept-reject steps described above, is summarized in Algorithm 5.

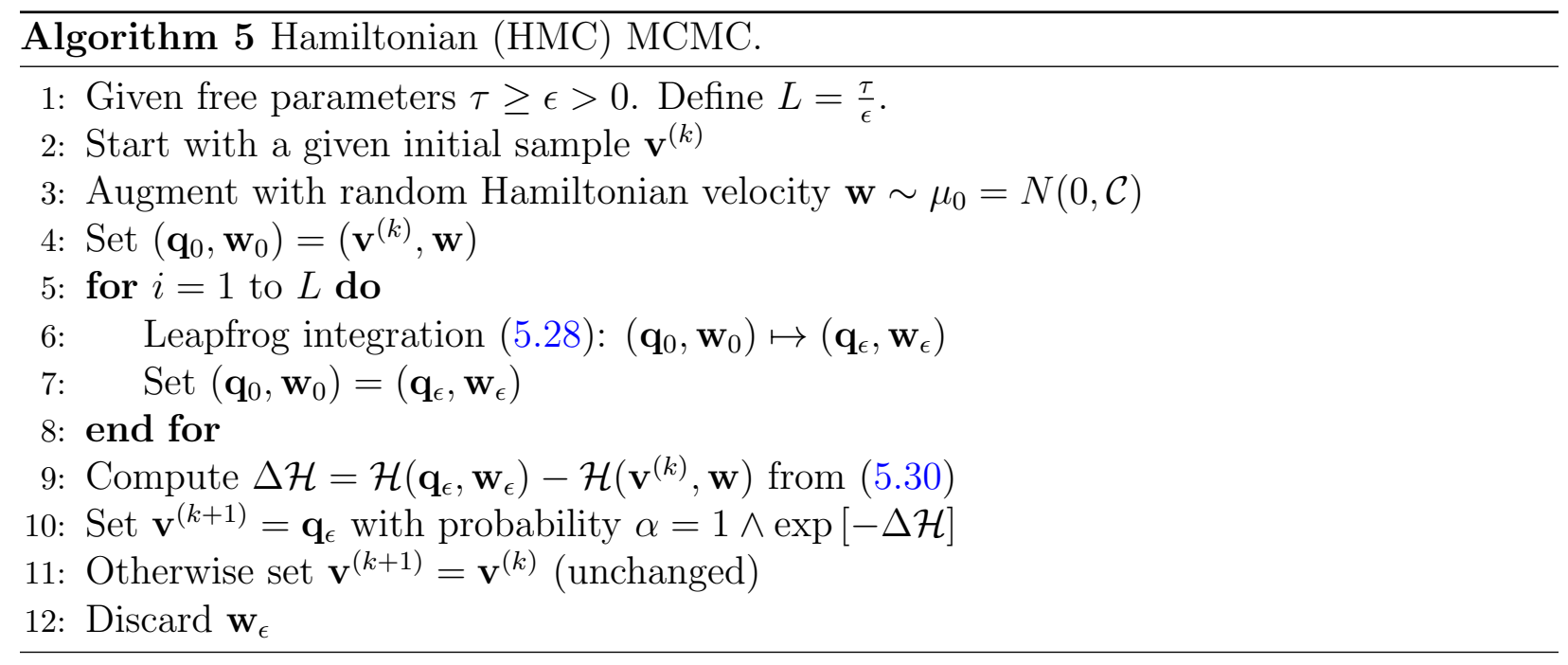

\subsection{Summary of MCMC Methods}

Table 5.1 summarizes the differences between the infinite-dimensional MCMC methods described above. A detailed investigation of their performance when applied to two different example problems is provided in Chapter 8.

Figure 5.2 diagrams the basic steps in a typical Metropolis-Hastings sampler. Note that HMC is somewhat more complicated, however, involving potentially many simulations of the PDE and computation of $\Phi$ and $D \Phi$ before the accept/reject step. The computation of the Fréchet derivative $D \Phi$ can be performed efficiently by solving an adjoint equation (see Section 6.2) or using automatic differentiation.[17, 32] 


\begin{tabular}{|l|l|l|l|l|}
\hline & IS & pCN & MALA & HMC \\
\hline \hline Applies to & Any Prior & Gaussian Prior & Gaussian Prior & Gaussian Prior \\
\hline Free Parameters & None & $\beta \in(0,1]$ & $h>0$ & $\epsilon>0, \tau>0$ \\
\hline $\begin{array}{l}\text { Proposals } \\
\text { Sampled From }\end{array}$ & Prior & Prior & Posterior & Posterior \\
\hline $\begin{array}{l}\text { Accept/Reject } \\
\text { Corrects for }\end{array}$ & $\begin{array}{l}\text { Prior to Poste- } \\
\text { rior }\end{array}$ & $\begin{array}{l}\text { Prior to Poste- } \\
\text { rior }\end{array}$ & $\begin{array}{l}\text { SDE Discretiza- } \\
\text { tion Errors }\end{array}$ & $\begin{array}{l}\text { PDE Discretiza- } \\
\text { tion Errors }\end{array}$ \\
\hline $\begin{array}{l}\text { Computational } \\
\text { Cost Per } \\
\text { MCMC Step }\end{array}$ & 1 PDE Solve & 1 PDE Solve & $\begin{array}{l}\text { PDE Solve +1 } \\
\text { Adjoint Solve }\end{array}$ & $\begin{array}{l}\frac{\tau}{\epsilon} \text { PDE Solves }+ \\
\frac{\tau}{\epsilon} \text { Adjoint Solves }\end{array}$ \\
\hline
\end{tabular}

Table 5.1: Comparison of MCMC Methods.

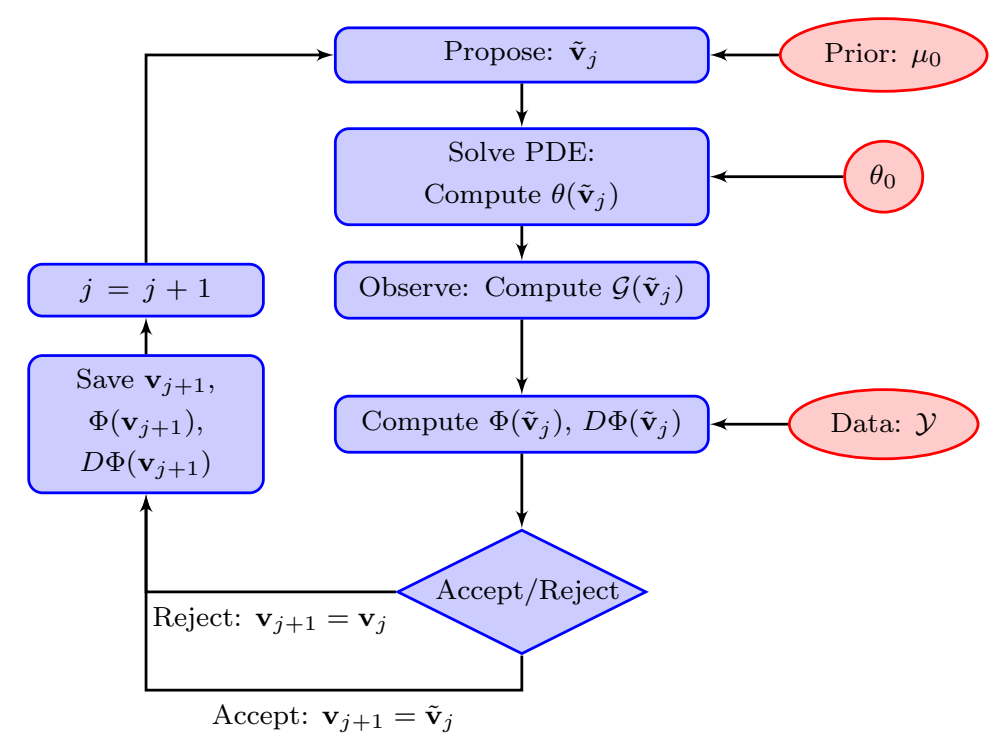

Figure 5.2: Diagram of Metroplis-Hastings MCMC. 


\section{Chapter 6}

\section{Practical and Theoretical}

\section{Implementation Challenges}

In this section, we will describe the numerical methods used to compute the quantities required by the MCMC algorithms for the advection-diffusion problem. In particular, we

outline the approach used to approximate the solution operator $\mathcal{S}$ (i.e., to compute $\theta$ from v) which, composed with the observation operator is used to approximate the forward map $\mathcal{G}$ (see Definition 3.6), and ultimately the potential $\Phi$ (see Definition 3.16). We also outline some significant computational challenges that arise in computing $\mathcal{S}$ for the advectiondiffusion problem for small $\kappa$, motivating the method outlined in Chapter 7 . We then define an adjoint method for efficient computation of the gradient of $\Phi$, a key ingredient in higherorder MCMC methods like MALA and HMC.

\subsection{Evaluation of $\mathcal{G}$}

Implementing the MCMC methods described in Chapter 5 requires evaluating the potential $\Phi$, which in turn requires evaluating $\mathcal{G}(\mathbf{v})$, i.e., computing the observations (e.g., point or spectral measurements) associated with a given vector field. This requires numerically solving (1.1) using a PDE solver, such as finite element or discontinuous Galerkin methods. In the numerical examples provided in Chapter 8 we use a Fourier solver, in which $\mathbf{v}$ is 
expanded in terms of $\mathbf{e}_{\mathbf{k}}$ as in (3.4) and $\theta$ is expanded similarly as $\theta(t, \mathbf{x})=\sum_{\mathbf{k}} \theta_{\mathbf{k}}(t) e^{2 \pi i \mathbf{k} \cdot \mathbf{x}}$. The coefficients are then written as system of ODEs

$$
\frac{d}{d t} \vec{\theta}(t)=A \vec{\theta}(t)
$$

and integrated using an implicit midpoint method to approximate $\vec{\theta}(t)$.

\subsubsection{Computational Challenges}

For any non-zero diffusion parameter $\kappa>0$, the long-time limit of the advection-diffusion equation is always the constant function $\theta(t, \mathbf{x})=\overline{\theta_{0}}$. However, the computational complexity involved in computing $\theta(t, \mathbf{x})$ (evaluating $\mathcal{G}$ ) can vary greatly with $\kappa$, which can range from $\mathcal{O}\left(10^{-1}\right)$ to $\mathcal{O}\left(10^{-5}\right)$ for chemicals diffusing in air and water, respectively [22]. When $\kappa$ is large, the diffusion quickly dominates, driving $\theta$ to the constant function. However, when $\kappa$ is small, the advection term quickly increases the number of non-zero components. In this case, accurately simulating $\theta$ can require including a substantial number of components, driving up computational time. To illustrate this point, Figure 6.1 shows the computed number of Fourier components $\theta_{\mathbf{k}}>0.001$ as a function of time for an example advectiondiffusion problem with a simple initial condition, for both $\kappa=3 \times 10^{-5}$ (chemical diffusing in water) and $\kappa=0.282$ (water molecule diffusing in air). The former case quickly consumes all available components in the simulated system $(\|\mathbf{k}\| \leq 32)$, while the latter quickly converges to a constant function. Accurate simulation of the low- $\kappa$ case is an active area of research $[20,45,61,62,81]$. Since MCMC methods can take many thousands of PDE solves to resolve some features of the posterior, this can present a substantial computational challenge. In computational examples provided in Chapter 8 , we will use either large $\kappa$ or short time intervals to keep the computational cost low enough to compute the many samples required 


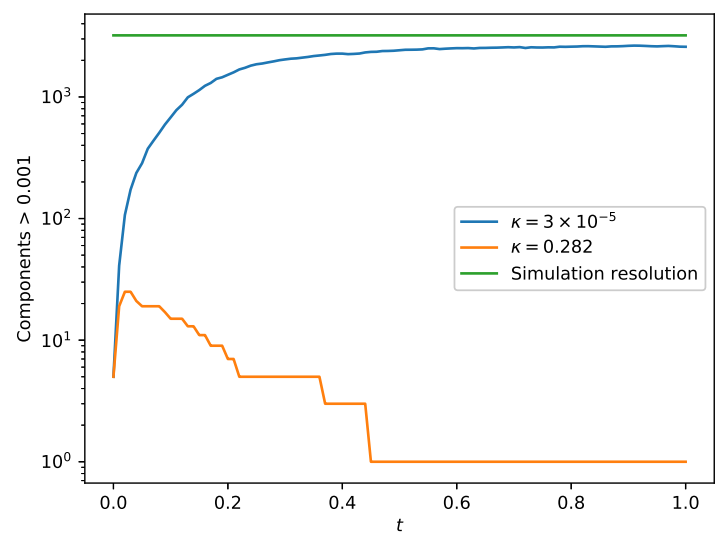

Figure 6.1: Number of Fourier components $\theta_{\mathbf{k}}>0.001$ for small and large $\kappa$.

to, for example, show histograms of the posterior.

In Chapter 7 we introduce a particle method for efficient computation of $\mathcal{G}(\mathbf{v})$. This will allow computation of large numbers of samples for more complex problems, such as low- $\kappa$ or high-turbulence vector fields.

\subsection{Adjoint Method for Evaluating the Gradient of $\Phi$}

Several MCMC algorithms, such as MALA (Section 5.4) or Hamiltonian (Section 5.5) Monte Carlo, require evaluating the Fréchet derivative of the potential $\Phi$ (see Definition 3.16) with respect to changes in $\mathbf{v}$ :

$$
D_{\tilde{\mathbf{v}}} \Phi(\mathbf{v}):=\lim _{\epsilon \rightarrow 0} \frac{1}{\epsilon}[\Phi(\mathbf{v}+\epsilon \tilde{\mathbf{v}})-\Phi(\mathbf{v})]
$$

Theorem 6.1 below describes an adjoint solution for evaluation of this derivative, which requires only (a forced version of) the same solver used to solve (1.1). For a detailed discussion of adjoint methods, we refer the reader to [44]. A similar adjoint equation was derived for a 
different application in [2] and a similar example is provided in [15].

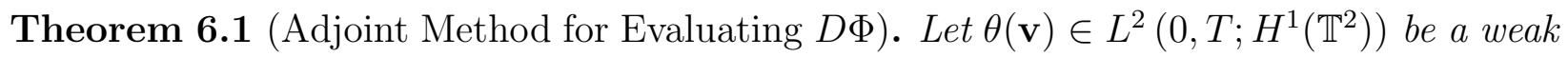
solution (see Definition 3.3) of the advection-diffusion equation (1.1) for background flow $\mathbf{v}$. Let $\mathcal{G}$ be the forward map and $\mathcal{O}$ be the observation operator (see Definition 3.6), and assume that $\mathcal{O}$ is linear and bounded. Suppose there exists a $\rho \in L^{2}\left([0, T] ; H^{1}\left(\mathbb{T}^{2}\right)\right)$ with $\rho(0)=0$ that solves the forced adjoint equation

$$
\int_{0}^{T}\left\langle\frac{\partial}{\partial t} \rho, \phi\right\rangle_{H^{-1}\left(\mathbb{T}^{2}\right) \times H^{1}\left(\mathbb{T}^{2}\right)}-\langle\mathbf{v} \cdot \nabla \rho, \phi\rangle_{L^{2}\left(\mathbb{T}^{2}\right)}+\kappa\langle\nabla \rho, \nabla \phi\rangle_{L^{2}\left(\mathbb{T}^{2}\right)} d t=-\frac{\partial \Phi}{\partial \mathcal{G}}(\mathbf{v}) \cdot \mathcal{O}[\tilde{\phi}]
$$

for all test functions $\phi \in L^{2}\left(0, T ; H^{1}\left(\mathbb{T}^{2}\right)\right)$, where $\tilde{\phi}(t, \mathbf{x}):=\phi(T-t, \mathbf{x})$. Then the Fréchet derivative of $\Phi$ at $\mathbf{v}$ in the direction $\tilde{\mathbf{v}}$ is given by

$$
D_{\tilde{\mathbf{v}}} \Phi(\mathbf{v})=\langle\tilde{\mathbf{v}} \cdot \nabla \theta, \tilde{\rho}\rangle_{L^{2}\left([0, T] \times \mathbb{T}^{2}\right)}
$$

where $\tilde{\rho}(t, \mathbf{x}):=\rho(T-t, \mathbf{x})$

Proof. Since the observation operator $\mathcal{O}$ is linear and bounded (and therefore continuous), we have

$$
\begin{aligned}
D_{\tilde{\mathbf{v}}} \mathcal{G}(\mathbf{v}) & =D_{\tilde{\mathbf{v}}} \mathcal{O}(\theta(\mathbf{v}))=\lim _{\epsilon \rightarrow 0} \frac{1}{\epsilon}[\mathcal{O}(\theta(\mathbf{v}+\epsilon \tilde{\mathbf{v}}))-\mathcal{O}(\theta(\mathbf{v}))]=\lim _{\epsilon \rightarrow 0} \mathcal{O}\left(\frac{1}{\epsilon}[\theta(\mathbf{v}+\epsilon \tilde{\mathbf{v}})-\theta(\mathbf{v})]\right) \\
& =\mathcal{O}\left(\lim _{\epsilon \rightarrow 0} \frac{1}{\epsilon}[\theta(\mathbf{v}+\epsilon \tilde{\mathbf{v}})-\theta(\mathbf{v})]\right)=\mathcal{O}\left[D_{\tilde{\mathbf{v}}}(\theta(\mathbf{v}))\right] .
\end{aligned}
$$

Then application of the chain rule yields

$$
D_{\tilde{\mathbf{v}}} \Phi(\mathbf{v})=\frac{\partial \Phi}{\partial \mathcal{G}}(\mathbf{v}) \cdot D_{\tilde{\mathbf{v}}} \mathcal{G}(\mathbf{v})=\frac{\partial \Phi}{\partial \mathcal{G}}(\mathbf{v}) \cdot \mathcal{O}\left[D_{\tilde{\mathbf{v}}} \theta(\mathbf{v})\right]
$$

Denote $D_{\tilde{\mathbf{v}}} \theta(\mathbf{v})$ by $\psi(\mathbf{v}, \tilde{\mathbf{v}})$. Then by applying the advection-diffusion equation (1.1) to 
$\theta(\mathbf{v}+\epsilon \tilde{\mathbf{v}})$ and $\theta(\mathbf{v})$, subtracting, taking the $\epsilon \rightarrow 0$ limit, and using the definition of the Fréchet derivative, we see that $\psi \in L^{2}\left(0, T ; H^{1}\left(\mathbb{T}^{2}\right)\right)$ satisfies

$$
\left\langle\frac{\partial}{\partial t} \psi, \phi\right\rangle_{H^{-1}\left(\mathbb{T}^{2}\right) \times H^{1}\left(\mathbb{T}^{2}\right)}+\langle\mathbf{v} \cdot \nabla \psi, \phi\rangle_{L^{2}\left(\mathbb{T}^{2}\right)}+\kappa\langle\nabla \psi, \nabla \phi\rangle_{L^{2}\left(\mathbb{T}^{2}\right)}=-\langle\tilde{\mathbf{v}} \cdot \nabla \theta, \phi\rangle_{L^{2}\left(\mathbb{T}^{2}\right)}
$$

with $\psi(0)=0$, for all test functions $\phi \in H^{1}\left(\mathbb{T}^{2}\right)$ and almost all times $t \in[0, T]$.

Replacing $t$ with $T-t$ in (6.2) yields the following relationship for $\tilde{\rho}(t)=\rho(T-t)$ :

$$
\int_{0}^{T}\left\langle\frac{\partial}{\partial t} \tilde{\rho}, \phi\right\rangle_{H^{-1}\left(\mathbb{T}^{2}\right) \times H^{1}\left(\mathbb{T}^{2}\right)}+\langle\mathbf{v} \cdot \nabla \tilde{\rho}, \phi\rangle_{L^{2}\left(\mathbb{T}^{2}\right)}-\kappa\langle\nabla \tilde{\rho}, \nabla \phi\rangle_{L^{2}\left(\mathbb{T}^{2}\right)} d t=\frac{\partial \Phi}{\partial \mathcal{G}}(\mathbf{v}) \cdot \mathcal{O}[\phi]
$$

with $\tilde{\rho}(T)=0$. Then, applying (6.5), (6.7), and (6.6) in succession yields

$$
\begin{aligned}
D_{\tilde{\mathbf{v}}} \Phi(\mathbf{v}) & =\frac{\partial \Phi}{\partial \mathcal{G}}(\mathbf{v}) \cdot \mathcal{O}[\psi] \\
& =\int_{0}^{T}\left\langle\frac{\partial}{\partial t} \tilde{\rho}, \psi\right\rangle_{H^{-1}\left(\mathbb{T}^{2}\right) \times H^{1}\left(\mathbb{T}^{2}\right)}+\langle\mathbf{v} \cdot \nabla \tilde{\rho}, \psi\rangle_{L^{2}\left(\mathbb{T}^{2}\right)}-\kappa\langle\nabla \tilde{\rho}, \nabla \psi\rangle_{L^{2}\left(\mathbb{T}^{2}\right)} d t \\
& =\int_{0}^{T}-\left\langle\tilde{\rho}, \frac{\partial}{\partial t} \psi\right\rangle_{H^{1}\left(\mathbb{T}^{2}\right) \times H^{-1}\left(\mathbb{T}^{2}\right)}-\langle\tilde{\rho}, \mathbf{v} \cdot \nabla \psi\rangle_{L^{2}\left(\mathbb{T}^{2}\right)}-\kappa\langle\nabla \tilde{\rho}, \nabla \psi\rangle_{L^{2}\left(\mathbb{T}^{2}\right)} d t \\
& =\langle\tilde{\mathbf{v}} \cdot \nabla \theta, \tilde{\rho}\rangle_{L^{2}\left([0, T] \times \mathbb{T}^{2}\right)} .
\end{aligned}
$$

Remark 6.2. Note that if the observation operator $\mathcal{O}$ can be written in terms of a kernel $K \in L^{2}\left(0, T ; H^{-1}\left(\mathbb{T}^{2}\right)\right)$ such that

$$
\mathcal{O}[\phi]=\int_{0}^{T}\langle K, \phi\rangle_{H^{-1}\left(\mathbb{T}^{2}\right) \times H^{1}\left(\mathbb{T}^{2}\right)} d t
$$

for all test functions $\phi \in L^{2}\left(0, T ; H^{1}\left(\mathbb{T}^{2}\right)\right)$, then solving (6.2) amounts to finding the weak 
solution of

$$
\frac{\partial}{\partial t} \rho(t, \mathbf{x})-\mathbf{v} \cdot \nabla \rho(t, \mathbf{x})-\kappa \Delta \rho(t, \mathbf{x})=-\frac{\partial \Phi}{\partial \mathcal{G}}(\theta(t, \mathbf{x}, \mathbf{v})) \cdot K(T-t, \mathbf{x}), \quad \rho(0, \mathbf{x})=0
$$

Example 6.3. Let $\eta_{j} \sim N\left(0, \sigma_{\eta}^{2}\right)$ for $j=1, \ldots, N$ so that $\Phi$ is given by (see Equation (3.27))

$$
\Phi(\mathbf{v} ; \mathcal{Y})=\sum_{j=1}^{N} \frac{1}{2 \sigma_{\eta}^{2}}\left(\mathcal{Y}_{j}-\mathcal{G}_{j}(\mathbf{v})\right)^{2}
$$

Let the observation operator be point observations given by

$$
\mathcal{O}_{j}[\theta]=\theta\left(t_{j}, \mathbf{x}_{j}\right)
$$

Note that these observations are linear and continuous by Corollary 3.10. Then the observation kernel (6.9) is given by

$$
K_{j}(t, \mathbf{x})=\delta\left(t-t_{j}\right) \delta\left(\mathbf{x}-\mathbf{x}_{j}\right)
$$

where $\delta$ is the Dirac delta function. Then solving (6.2) amounts to finding the weak solution of

$$
\frac{\partial}{\partial t} \rho(t, \mathbf{x})-\mathbf{v} \cdot \nabla \rho(t, \mathbf{x})-\kappa \Delta \rho(t, \mathbf{x})=\sum_{j} \frac{1}{\sigma_{\eta}^{2}}\left(\mathcal{Y}_{j}-\theta\left(t_{j}, \mathbf{x}_{j}, \mathbf{v}\right)\right) \delta\left(T-t_{j}-t\right) \delta\left(\mathbf{x}-\mathbf{x}_{j}\right)
$$

where $\rho(0, \mathbf{x})=0$.

To compute the full gradient $D \Phi$, we compute the integration (6.3) for $\tilde{\mathbf{v}}=\mathbf{e}_{\mathbf{k}}$ for each $\mathbf{k}$. The resulting algorithm is summarized in Algorithm 6.

Note that solving (6.6) and plugging into (6.5) would also yield the derivative of $\Phi$. However, 
this approach would require a PDE solve for each direction $\tilde{\mathbf{v}}$ in which we want to take the derivative. In particular, if we want the full gradient (the derivative with respect to an array of bases $\left\{\mathbf{e}_{\mathbf{k}}\right\}$ ), we have to do many PDE solves. By contrast, Algorithm 6 requires only one additional PDE solve per gradient calculation.

Moreover, note that (6.2) is equivalent to (1.1) with zero initial condition, a reversed vector field, and a forcing term. Thus, the same PDE solver can be used for both the forward and adjoint solves with minimal modification.

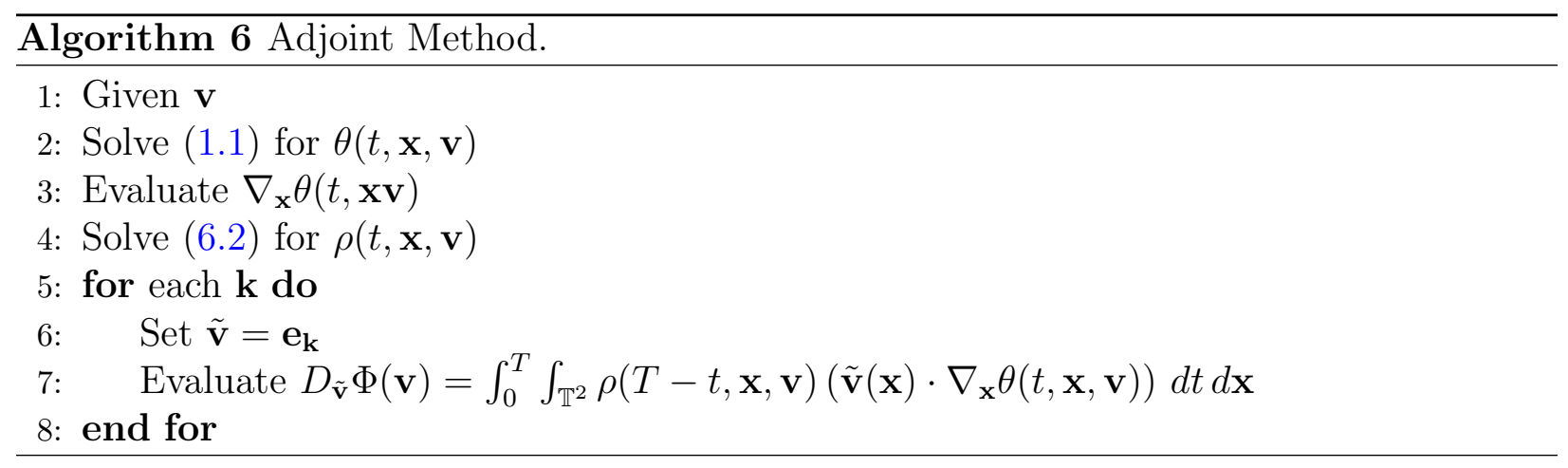




\section{Chapter 7}

\section{A Particle Method for Sparse Point Observations}

As described in Section 6.1, a straightforward method for evaluating $\mathcal{G}$ involves two steps:

1. Compute $\theta(\mathbf{v})$ via some numerical PDE solver

2. Compute observations $\mathcal{G}(\mathbf{v})$ from $\theta(\mathbf{v})$

This natural approach has the benefit of allowing the application of third-party "black-box" PDE solvers to the inverse problem.

However, computing $\mathcal{S}(\mathbf{v})$ involves approximating a solution that is infinite-dimensional, which can be very computationally expensive, requiring the evaluation of a PDE for many thousands or millions of basis functions. By contrast, the evaluation of $\mathcal{O}$ then involves projecting that PDE solution into a finite-dimensional space. As a result, much of the work involved in approximating $\mathcal{S}$ is, in some sense, discarded in the application of $\mathcal{O}$.

In this chapter, we present a numerical method that breaks this two-step paradigm by allowing computation of point observations of $\theta$ directly, without computation of the full solution. This method therefore bypasses the need to approximate a high-dimensional PDE solution at each step of the inverse problem, instead replacing the full PDE solve with an array of particle solutions that are less computationally expensive. Moreover, because the 
particle simulations are decoupled, they can be parallelized in a straightforward manner on modern computational architectures. The result is a dramatic speedup, particularly for problems in which the dimension of the unknowns is much larger than the dimension of the observations.

\subsection{Method}

In this section, we present a particle method that will allow point evaluation of $\theta$ directly from the unknown $\mathbf{v}$ without separate approximation of $\mathcal{S}$. The method will involve simulating an ensemble of particles (Itô diffusions), which is a well-known method for approximating $\theta \cdot[19,25]$

However, to leverage the sparse nature of the observations, for this application we will simulate the particles backward in time from their final condition to their initial condition. Doing so will allow us to avoid computing the entire field $\theta$ by computing it only where it is needed. A key ingredient is Kolmogorov's Backward Equation, which was introduced in Theorem 3.7 .

Kolmogorov's Backward Equation tells us that the value of $\theta$ at a particular time and location $(t, \mathbf{x})$ is given by the average value at time $t$ of the Itô diffu-

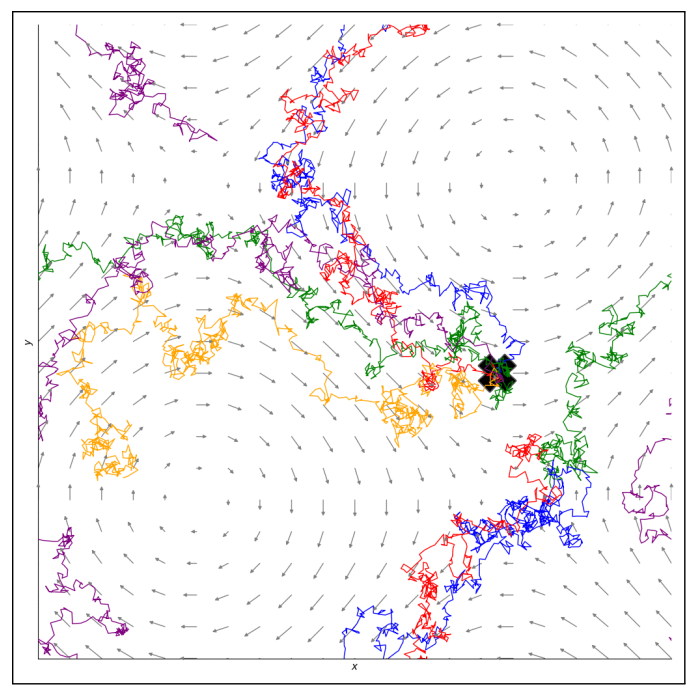

Figure 7.1: Traces of 5 simulated Itô diffusions with the same final position.

sion (3.11) initialized at $\mathbf{x}$. This suggests a numerical

method for evaluating $\mathcal{G}_{j}(\mathbf{v})=\theta\left(t_{j}, \mathbf{x}_{j}, \mathbf{v}\right)$ : (1) initialize a series of particles from $\mathbf{x}_{j} ;(2)$ simulate their movement to time $t_{j}$ according to $(3.11)$; (3) evaluate $\theta_{0}$ at that location; and 
(4) take the average.

Numerical integration of (3.11) can be computed, for example, with an Euler-Maruyama or Millstein approximation (which are equivalent for spatially-constant $\kappa$ ) $[43,47]$

$$
\mathbf{X}_{i+1}=\mathbf{X}_{i}-\mathbf{v}\left(\mathbf{X}_{i}\right) \Delta t_{i}+\sqrt{2 \kappa \Delta t_{i}} \xi_{i}
$$

where $\mathbf{X}_{i}=\mathbf{X}\left(t_{i}\right), \Delta t_{i}=t_{i+1}-t_{i}$, and $\xi_{i} \sim N(0,1)$. The resulting algorithm is described in Algorithm 7, where $N_{o}$ is the number of observations and $N_{p}$ is the number of particles used per observation.

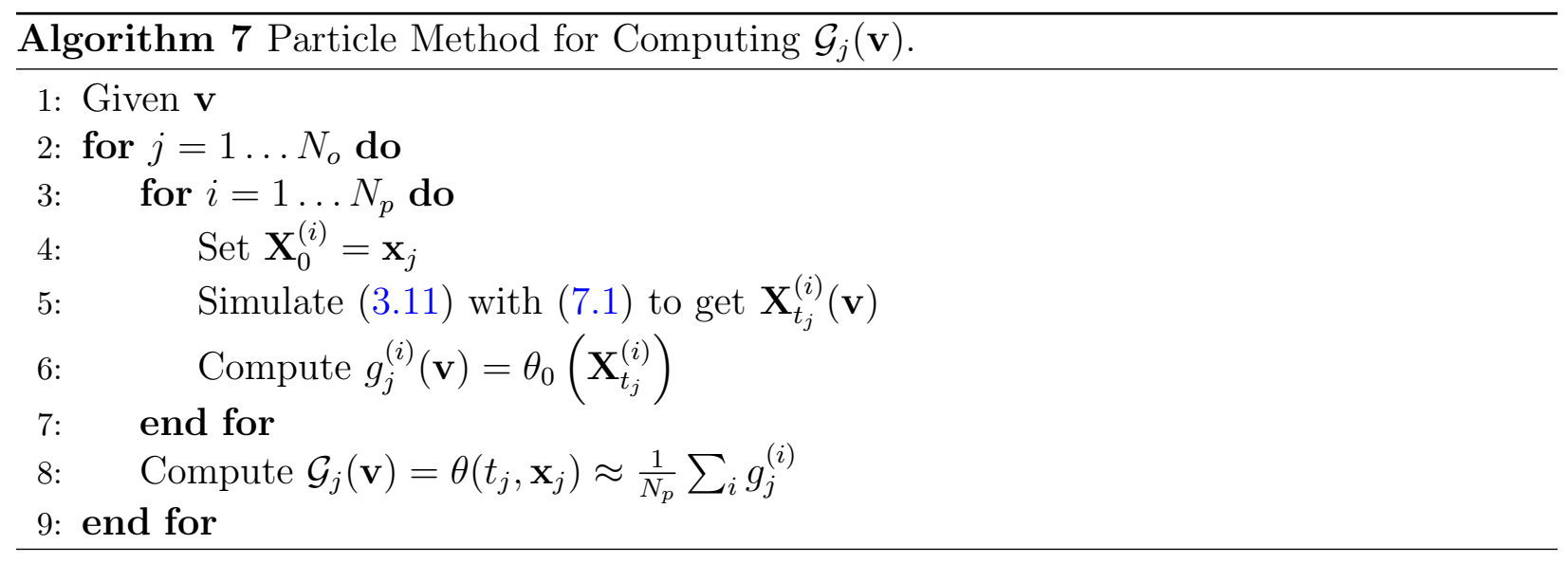

\subsection{Parallelization}

Note that for a given observation point $\left(t_{j}, \mathbf{x}_{j}\right)$, all but the final step of Algorithm 7 can be computed in parallel. In addition, the steps for separate observation points are entirely independent. As a result, the algorithm is largely embarrassingly parallel and can therefore be parallelized in a straightforward manner using any number of computational paradigms, such as message passing interface (MPI) processes or OpenMP threads, and naturally vectorizes to leverage "single instruction multiple data" (SIMD) capabilities on modern CPUs 
or GPUs.

For example, Figure 7.2, from NVIDIA's documentation, [67] illustrates the layout of threads and blocks on an NVIDIA GPU. Each thread is a single execution unit that is grouped into a block and then run across one or more SIMD. Algorithm 7 can be ported to this architecture in a natural fashion: each observation can be assigned to a block or group of blocks, with each particle run in a thread. A single GPU can execute thousands of threads at a time, allowing thousands of particles to be simulated simultaneously on a single chip. Moreover, larger problems can be spread across multiple GPUs or multiple machines with MPI to gain even greater efficiency; the overhead

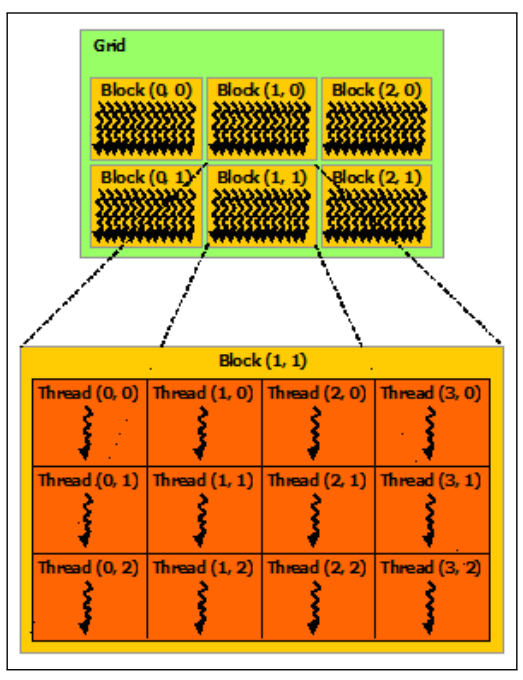

Figure 7.2: GPU thread blocks[67]. is minimal as only the average value for each observation need be returned to the master process.

\subsection{Computational Complexity}

In this section, we will compare the computational costs of the particle method with that of a reference Galerkin-based PDE solve. Consider the case where $\mathbf{v}$ is approximated by a basis expansion $\mathbf{v} \approx \sum_{i=1}^{N_{\mathbf{v}}} v_{i} \mathbf{e}_{i}$ (as in Section 3.1) and evaluation of each basis function $\mathbf{e}_{i}$ has computational cost $C_{b}$. We use the Euler-Maruyama approximation (7.1) and assume $N_{t}$ timesteps per observation. Further, we assume evaluating $\mathcal{G}$ requires $N_{o}$ observations and use $N_{p}$ particles per observation. Then the computational cost of Algorithm 7 for $P$ parallel 
processes/threads is given by

$$
C_{\text {particle }}=O\left(\frac{1}{P} N_{o} N_{p} N_{t} N_{\mathbf{v}} C_{b}\right)
$$

Since the observations and particle simulations are almost entirely independent, they can be executed in parallel (see Section 7.2). So for large $P$ we have the limit

$$
C_{\text {particle }} \rightarrow O\left(N_{t} N_{\mathbf{v}} C_{b}\right)
$$

A more traditional method of solving the PDE would be to use a Galerkin projection, as in Section 6.1, which would involve projecting the PDE (1.1) onto a set of basis functions $\{\phi\}_{l=1}^{N_{b}}$ to get a system of ODEs:

$$
\begin{aligned}
M \dot{\vec{\theta}} & =A \vec{\theta} \\
M_{l m} & =\left\langle\phi_{l}, \phi_{m}\right\rangle \\
A_{l m} & =\left\langle\phi_{l},-\mathbf{v}(\mathbf{x}) \cdot \nabla \phi_{m}+\kappa \Delta \phi_{m}\right\rangle .
\end{aligned}
$$

The bases $\phi_{l}$ could, for example, be Fourier or finite element basis functions. The system (7.4) is then integrated by repeated iteration of some combination of $M, A$ (explicit, Runge-Kutta methods) and/or their inverses (implicit methods). Algorithm 8 outlines this algorithm for Explicit Euler time integration.

The computational costs of assembling the matrix $A$ are heavily dependent on the choice of $\mathbf{v}$ and $\{\phi\}$ and therefore difficult to characterize in general. The cost of computing the observations is typically small. Ignoring these two factors, the computational cost of Algorithm 8 is dominated by the time integration of the system, which is made up of a series of matrix-vector multiplications. In general, the cost of this computation for $N_{b}$ basis 


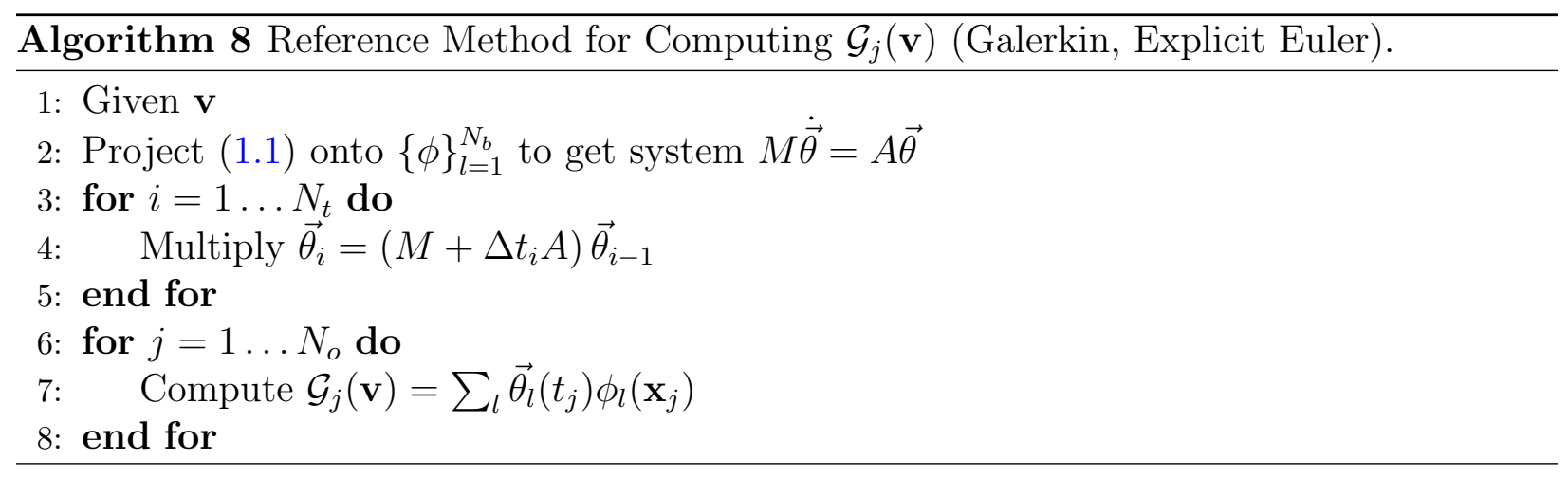

functions and $N_{t}$ time steps is

$$
C_{\text {reference }} \rightarrow O\left(N_{t} N_{b}^{2}\right)
$$

Note also that to model an unknown of dimension $N_{\mathbf{v}}$ requires $N_{b} \geq N_{\mathbf{v}}$. In practice, however, accurate modeling of $\theta$ may require $N_{b} \gg N_{\mathbf{v}}$, particularly for small diffusion. Thus the ratio of the cost of the particle method (7.2) to the reference method (7.5) is

$$
\frac{C_{\text {particle }}}{C_{\text {reference }}} \leq O\left(\frac{C_{b}}{N_{b}}\right)
$$

Thus for applications with small enough number of observations or sufficient parallelism that a substantial proportion of the particles can be computed in parallel, the particle method should provide substantial speedup for large $N_{b}$ and in particular for problems with highdimensional unknowns.

We have of course, made some simplifications in this analysis: For some choices of $\{\phi\}$ (e.g., discontinuous Galerkin[42]), the matrix $A$ may be sparse or block diagonal, reducing the cost of the matrix multiply and increasing the effectiveness of parallelization. However, we have also ignored some of the costs of Algorithm 8. Most of the performance improvement comes from not computing the full field $\theta$, so the computational complexity for large $N_{\mathbf{v}}$ should be 
as indicated here for a large class of reference algorithms.

\subsection{Limitations}

For completeness, we now identify two key limitations of using Algorithm 7 to compute $\mathcal{G}$ :

- It is sometimes desirable to compute charateristics of $\theta$ beyond what is contained in $\mathcal{G}$. For example, in Chapter 8 we present the statistics of the variance and variance dissipation of $\theta$ according to the Bayesian posterior on $\mathbf{v}$. Of course, because Algorithm 7 does not involve computing the full field $\theta$, we cannot compute characteristics of $\theta$ beyond the values contained in $\mathcal{G}$. However, we note that the proposed particle algorithm could be used to compute the solution to the inverse problem, e.g., the maximum a posteriori $\mathbf{v}_{M A P}$. Then a single, computationally-expensive PDE solve could be used to compute the characteristics of $\theta\left(\mathbf{v}_{M A P}\right)$.

- Many approaches to inverse methods require computing the Fréchet derivative $D \mathcal{G}(\mathbf{v})$, which cannot be computed via the particle method in its current form. The gradient of $\mathcal{G}$ would therefore have to be approximated via a finite-difference approximation involving multiple computations of $\mathcal{G}$. 


\section{Chapter 8}

\section{Numerical Experiments}

In this section, we describe applications of the above methods to two sample problems. We begin by introducing a prior measure that may be useful to passive scalar communities, which we then use in each example. We then provide an example problem (Section 8.2) that yields a posterior measure with a relatively simple structure. We show that many different MCMC methods are successful in sampling from this posterior. We then introduce a second example (Section 8.3) for which the posterior measure exhibits an extremely complicated, multiwelled structure. This example is more challenging for MCMC methods to sample from, and thus a good discriminator between the simple and more advanced approaches introduced in Chapter 5. We also use this second example as a test case for the particle method (Section 8.3.6), extending the inversion from hundreds to thousands of dimensions. Finally, we provide a summary of the lessons learned from these two examples and implications for practitioners of MCMC methods.

In each example, we generate data $\mathcal{Y}$ by running a high-resolution simulation of the system for a given true vector field $\mathbf{v}^{\star}$ and then applying the observation operator $\mathcal{O}$. $\mathbf{v}^{\star}$ is the "true" vector field; in an ideal scenario we could invert the problem exactly to obtain it. However, as described in Section 3.3 we will not be able to do so in general.

Each of the examples, including the PDE solvers, adjoint solver, and MCMC methods, is implemented in the Julia numerical computing language [13] version 0.5.2. The particle method is written in a combination of $\mathrm{C}$ and CUDA. Thousands, or in some cases millions, 
of samples were generated using the computational resources at Virginia Tech; the particle computations in Section 8.3.6 used a single NVIDIA P100 GPU. We note that a multi-GPU implementation should yield further speedup for larger problem sizes, with minimal overhead due to the embarrassingly parallel nature of the particle method.

\subsection{A Prior for Passive Scalar Problems}

A key idea in the modeling of passive scalars in turbulent flows is the Kraichnan model [50], which models turbulent advection with a Gaussian random velocity field with a given energy spectrum (see, for example, equation 28 of [18])

$$
E(k)=E_{0} \sum_{i=0}^{N}\left(\frac{k}{k_{i}}\right)^{4} \exp \left[-\frac{3}{2}\left(\frac{k}{k_{i}}\right)^{2}\right] k_{i}^{-\xi}
$$

where $k_{i}=\sqrt{2}^{i}$ is the characteristic wave number of the $i$ th subfield, $N$ is the number of subfields, and $E_{0}$ controls the overall energy. The resulting spectrum exhibits $E(k) \propto k^{-\xi}$ for $1<k<k_{N}=2^{N / 2}$ and exponential decay for $k>k_{N}$; both of these features are shown in Figure 8.1. Note that when $\mathbf{v}$ is the solution of the two-dimensional Navier-Stokes equation, the cutoff wave number $k_{N}$ satisfies $k_{N}=\chi^{-\frac{1}{6}} \nu^{-\frac{1}{2}}$ where $\chi$ is the rate of cascade of mean-square vorticity and $\nu$ is the kinematic viscosity of the fluid; i.e., the power law range consumes a larger range of wave numbers for less viscous fluids. [6, 49]

A reasonable prior may then be

$$
\mu_{0}=N(0, \tilde{E})
$$

where $\tilde{E}_{i j}=\frac{1}{2 \pi\left\|\mathbf{k}_{i}\right\|_{2}} E\left(\left\|\mathbf{k}_{i}\right\|_{2}\right) \delta_{i j}$. Then for $\mathbf{v}=\sum_{\mathbf{k}} v_{k} \sim \mu_{0}$, the expected energy associated 
with wave number $k$ is

$$
\mathbb{E} \int_{S_{k}}\left|v_{\mathbf{k}}\right|^{2}=\int_{S_{k}} \mathbb{E}\left|v_{\mathbf{k}}\right|^{2}=\int_{S_{k}} \tilde{E}(k)=\int_{S_{k}} \frac{1}{2 \pi k} E(k)=E(k)
$$

where $S_{k}=\left\{\mathbf{k}:\|\mathbf{k}\|_{2}=k\right\}$ is the shell associated with wave numbers of norm $k$. The energy decay is shown in Figure 8.2; integrals of this surface along circles with radius $\|\mathbf{k}\|_{2}$ will yield the values shown in Figure 8.1.

In the examples in this section, we will use $\mu_{0}$ as defined in (8.2) for $E$ as defined in (8.1) with $\xi=\frac{3}{2}$, motivated by $[18,51]$. An example vector field drawn randomly from this prior (with $\|\mathbf{k}\|_{2} \leq 32$ ) is shown in Figure 8.3.

Note: The Kraichnan model of mixing typically involves a velocity field with energy spectrum (8.1) but that is white ( $\delta$-correlated) in time.[78] Here $\mathbf{v}$ is a background flow, i.e. constant in time; we simply use the Kraichnan model as motivation for the energy decay modeled in the prior.
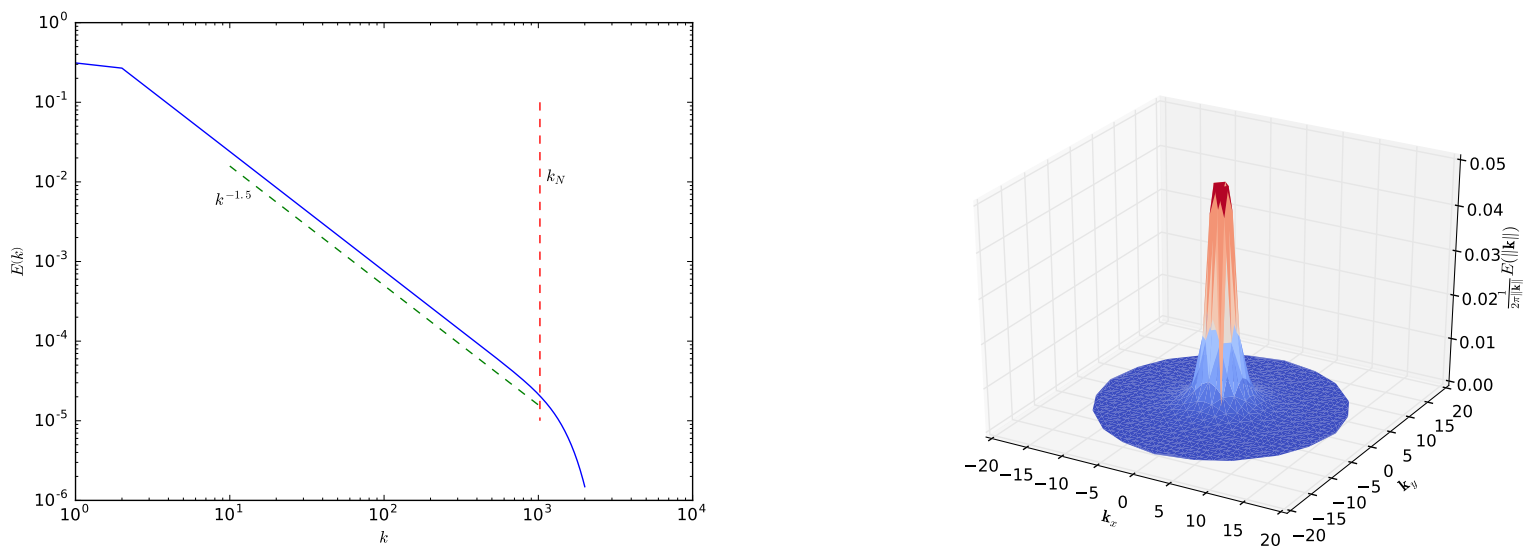

Figure 8.1: The Kraichnan energy spectrum

$E(k)$ (8.1) in one dimension (compare with Figure 8.2: The Kraichnan energy spectrum Figure 6 from [18]). $\frac{1}{2 \pi\|\mathbf{k}\|_{2}} E\left(\|\mathbf{k}\|_{2}\right)$ in two dimensions. 


\subsection{Example 1: Single-welled Posterior}

In this subsection, we construct an example that yields a posterior distribution with a simple, single-welled structure. This example will be relatively easy for several MCMC methods to sample from with a single chain. The problem parameters for this example are enumerated in Table 8.1. ${ }^{1}$ The true vector field $\mathbf{v}^{\star}$ is shown in Figure 8.3.

\begin{tabular}{|l|l|l|}
\hline Parameter & Symbol & Value \\
\hline \hline Observation operator & $\mathcal{O}$ & Point observations at 1,024 uniformly random $(t, x, y)$ \\
\hline Observed data & $\mathcal{Y}$ & $\mathcal{G}\left(\mathbf{v}^{\star}\right)$ for some $\mathbf{v}^{\star}$ \\
\hline True vector field & $\mathbf{v}^{\star}$ & Randomly drawn from Kraichnan prior, $\|\mathbf{k}\|_{2} \leq 32$ \\
\hline Sampling space & $H_{N}$ & $\|\mathbf{k}\|_{2} \leq 8(197$ components $)$ \\
\hline Prior distribution & $\mu_{0}$ & Kraichnan (see Section 8.1$)$ \\
\hline Diffusion parameter & $\kappa$ & 0.282, for water diffusing in air $[22]$ \\
\hline $\begin{array}{l}\text { Observational noise } \\
\text { distribution }\end{array}$ & $\mathbb{Q}_{0}$ & $N\left(0, \sigma_{\eta} I\right), \sigma_{\eta}=2^{-6}$ \\
\hline Initial condition & $\theta_{0}$ & $\frac{1}{2}-\frac{1}{4} \cos (2 \pi x)-\frac{1}{4} \cos (2 \pi y)$ \\
\hline
\end{tabular}

Table 8.1: Problem parameters for Example 1.

\subsubsection{Posterior Structure}

As described above, the output of a Bayesian inference is the posterior $\mu_{\mathcal{Y}}$, a probability measure on the space of divergence-free vector fields $H$ or, in practice, on a finite-dimensional approximation $H_{N}$ given by the truncated expansion of the basis described in Section 3.1. To approximate the exact posterior, we assembled a list of 10 million samples by running a series of $40 \mathrm{pCN}$ MCMC chains of 250,000 samples each, each beginning with a randomly-chosen

\footnotetext{
${ }^{1}$ Note that $\mathbf{v}^{\star}$ and observation locations/times were chosen randomly only to generate the scenario and data, with the random selection just used to ensure a sufficiently general problem. The data and observation operator then of course remain fixed during MCMC sampling.
} 

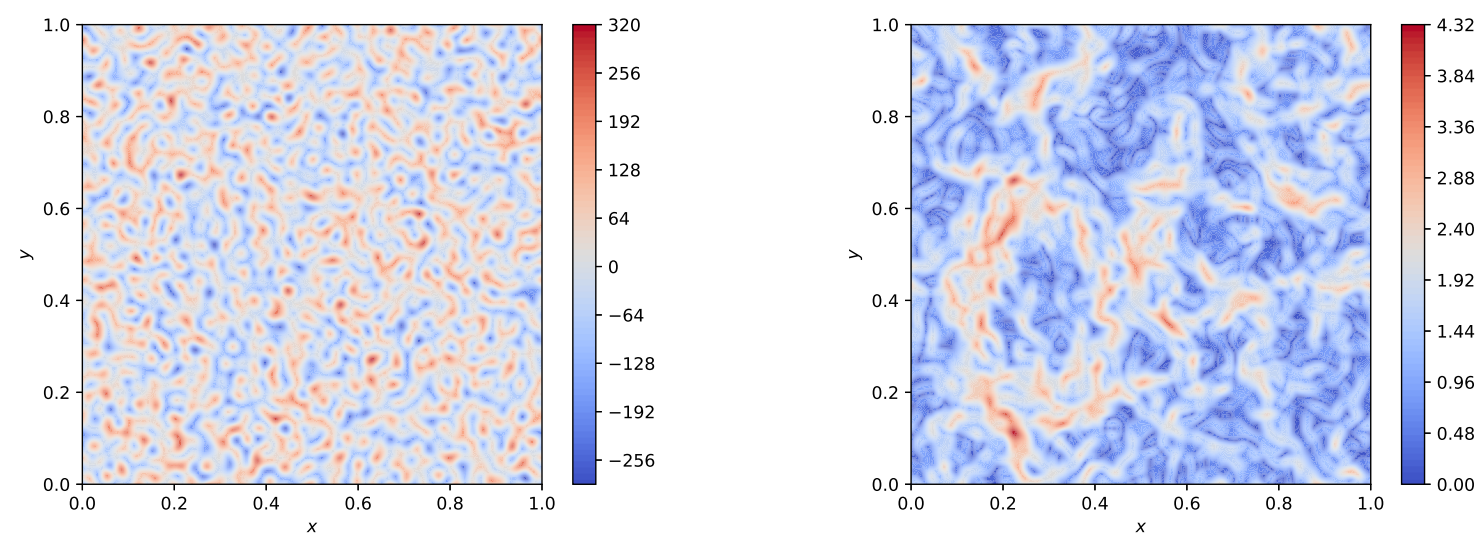

Figure 8.3: $\mathbf{v}^{\star}$ for Example 1. Left: Vorticity $\nabla \times \mathbf{v}^{\star}$, Right: $\left\|\mathbf{v}^{\star}\right\|$.

initial sample. (For a discussion of why multiple chains were used, see Section 8.3.)

Figure 8.4 presents histograms of the first few components of $\mathbf{v}$, where $\mathbf{v}$, incorporating the discretization and reality condition in (3.3), is constructed from the components as:

$$
\begin{aligned}
\mathbf{v}(\mathbf{x})= & {\left[v_{0}, v_{1}\right]+v_{2}[0, \cos (2 \pi y)]+v_{3}[0,-\sin (2 \pi y)]+v_{4}[\cos (2 \pi x), 0]+v_{5}[-\sin (2 \pi x), 0] } \\
& +v_{6}[0, \cos (4 \pi y)]+v_{7}[0,-\sin (4 \pi y)]+v_{8}[\cos (2 \pi x), \cos (2 \pi y)] \\
& +v_{9}[-\sin (2 \pi x),-\sin (2 \pi y)]+\ldots
\end{aligned}
$$

The histograms are normalized so that each represents the marginal probability density function of the component, according to posterior. For comparison, the density according to the prior is also shown (note: components with the same $\|\mathbf{k}\|_{2}$ have the same prior PDF, according to (8.1)). Two-dimensional histograms representing the approximate posterior joint probability density of pairs of vector field components are shown in Figure 8.5.

Note that each of these figures (and other plots omitted for brevity) show a contiguous mass of probability, indicating that one "class" of vector field matches both the prior and the data. For posteriors with more complicated structure, separate probability "humps" in 


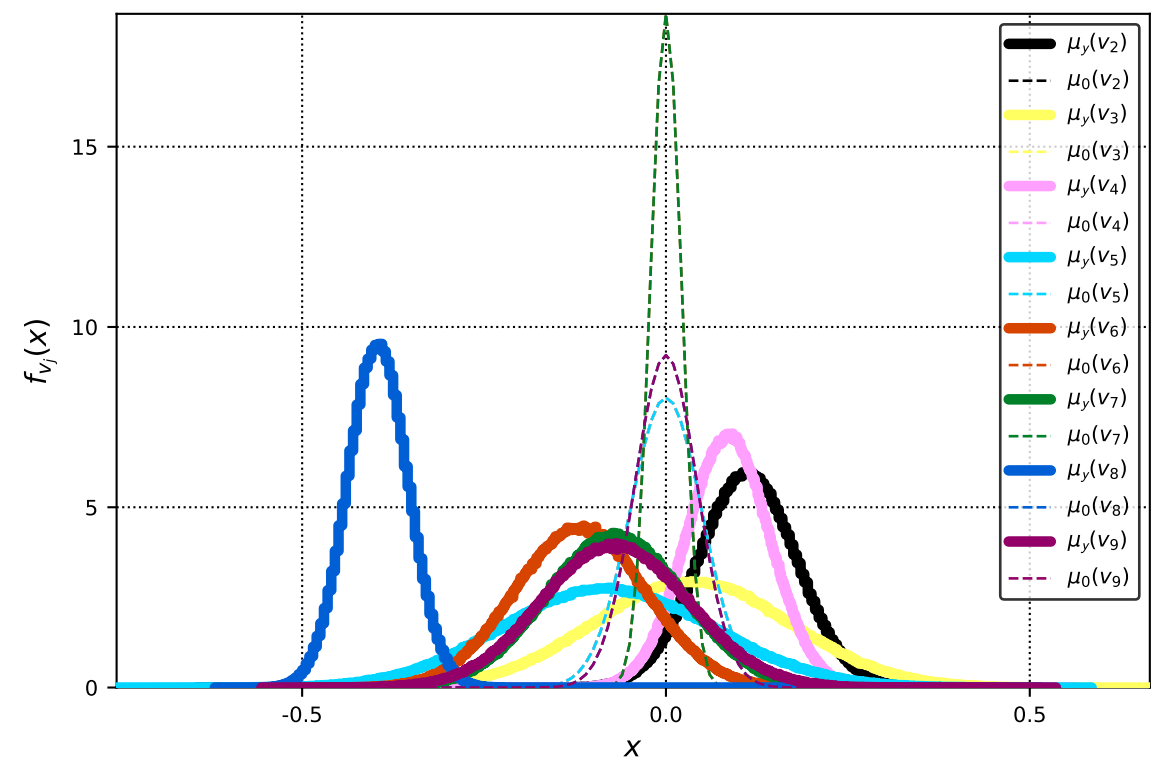

Figure 8.4: Prior $\left(\mu_{0}\right)$ and posterior $\left(\mu_{\mathcal{Y}}\right)$ marginal distributions for the first eight components of $\mathbf{v}$.

the posterior can also be identified, representing disparate classes of vector fields considered likely according to the posterior; Section 8.3 provides one such example. 


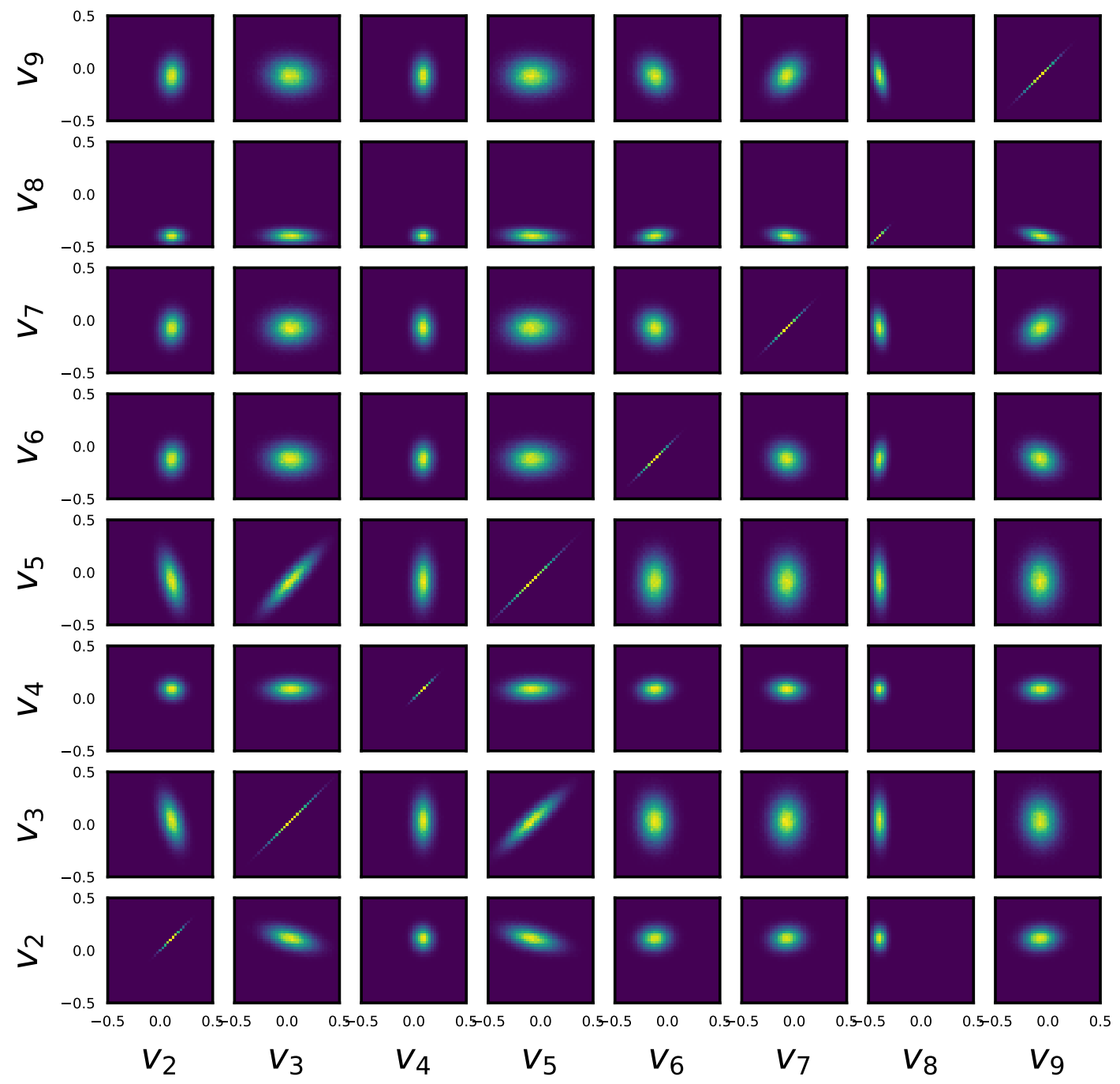

Figure 8.5: Posterior two-dimensional histograms for the first eight components of $\mathbf{v}$. 


\subsubsection{MCMC Sampling}

Each of the four MCMC methods introduced in Chapter 5 were applied to Example 1. Parameters used in the testing are listed in Table 8.2. The values for $\mathrm{pCN} \beta$ and MALA $h$ were chosen to match optimal acceptance rates from [71]. The values for HMC were chosen to yield high acceptance rate $(\epsilon)$ but still ensure large jumps $(\tau)$. A thorough explanation of parameter selection is provided in Section 8.3.2. The resulting acceptance ratios are shown in Table 8.3. As shown, the acceptance ratio for the independence sampler is very low even for a posterior with this relatively simple structure. MALA's $h$ parameter is small because the dimension of the data tends to make the gradient of $\Phi$ larger, which tends to make the jumps larger; this forced the choice of a small $h$ in order to avoid a large number of rejections. HMC avoids this limitation by using multi-step integration, allowing the user to tune both the step size via $\epsilon$ and the desired jump size via $\tau$.

\begin{tabular}{|c|c|c|}
\hline Method & Parameter & Value \\
\hline \hline $\mathrm{pCN}$ & $\beta$ & 0.150 \\
\hline MALA & $h$ & 0.005 \\
\hline HMC & $\epsilon$ & 0.125 \\
\hline HMC & $\tau$ & 1.000 \\
\hline
\end{tabular}

Table 8.2: MCMC Parameters Applied to Example 1.

\begin{tabular}{|c|c|}
\hline Method & Acceptance Ratio \\
\hline \hline IS & 0.00012 \\
\hline pCN & 0.23952 \\
\hline MALA & 0.53747 \\
\hline HMC & 0.81037 \\
\hline
\end{tabular}

Table 8.3: MCMC Acceptance Rates for Example 1. 
Figure 8.6 shows the trace and autocorrelation of the potential $\Phi$ (see (3.27)). Example 1 presents a case where the data are informative, i.e. the data disagree with the prior enough that the prior is not, in general, a good approximation of the posterior. As a result, when the Independence Sampler (see Section 5.2) is applied to this example, it generates proposals that are almost always rejected by the Metropolis-Hastings step. The acceptance ratio in this case was $0.012 \%$ and the chain concluded with thousands of consecutive rejections. Clearly, to get robust convergence, we need a method that incorporates either the previous sample (like pCN) or the data (like MALA and HMC) when generating proposals.

When either pCN or MALA are applied to this example, we see "random walk" behavior the samples move about the posterior, but are correlated with each other. MALA's samples are highly-correlated in this case - more highly than pCN despite the incorporation of a gradient computation - due to MALA's use of a small $h$. For HMC, the random walk effect is reduced. Samples exhibit independence from each after orders of magniture fewer iterations than for pCN or MALA. The chain explores the posterior more quickly as a result, as we will show in the next section.
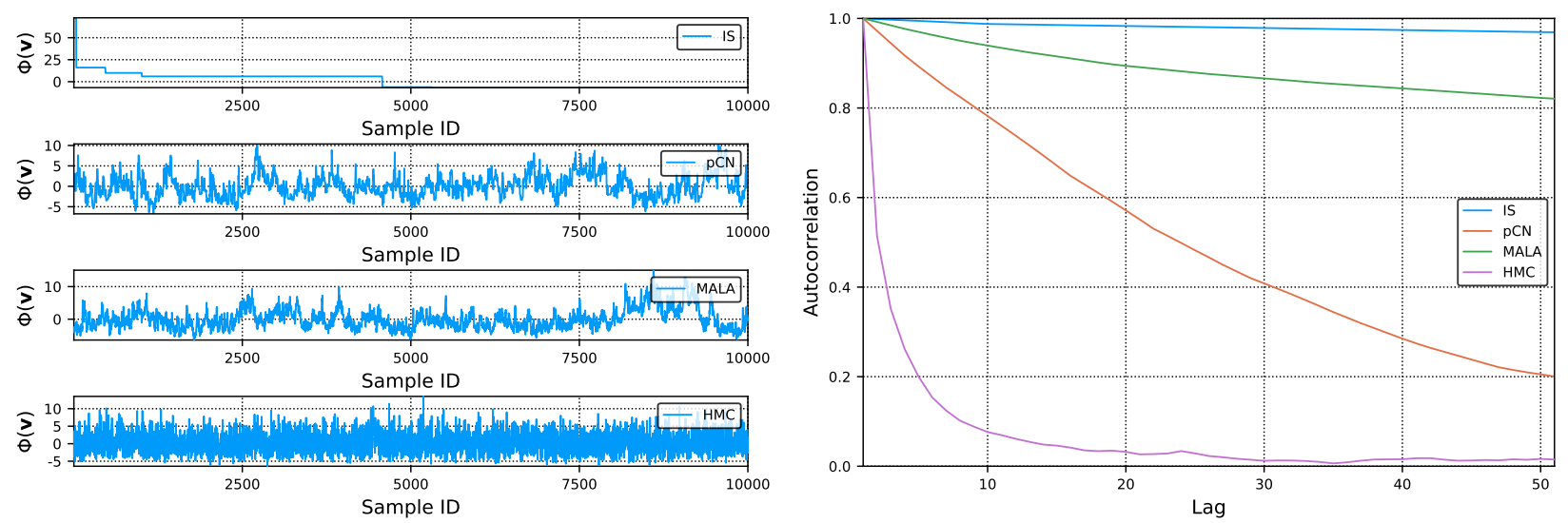

Figure 8.6: Trace (left) and autocorrelation (right) of the potential $\Phi$. 


\subsubsection{Convergence of Observables}

We will now compare convergence of features of the posterior as computed using various MCMC methods. In each case, the 40 pCN chains shown above will be used as the "true" posterior for comparison. We choose a series of observables involving both $\mathbf{v}$ and $\theta$ that are of interest to the passive scalar community, summarized in Table 8.4. Figure 8.7 shows the

\begin{tabular}{|c|c|}
\hline Observable & Formula \\
\hline \hline Mean Scalar Variance[84] & $\|\theta-\bar{\theta}\|_{L^{2}}^{2}$ \\
\hline Mean Scalar Dissipation Rate[78, 84] & $\epsilon_{\theta}=2 \kappa\|\nabla \theta\|_{L^{2}}^{2}$ \\
\hline Enstrophy [5, 52] & $\frac{1}{2}\|\nabla \times \mathbf{v}\|_{L^{2}}^{2}$ \\
\hline Enstrophy Dissipation Rate & $\|\nabla(\nabla \times \mathbf{v})\|_{L^{2}}^{2}$ \\
\hline
\end{tabular}

Table 8.4: Observables.

relative error in the mean value of each of the observables in Table 8.4 at $t=1$, through 10,000 samples. ${ }^{2}$ pCN and MALA converge at similar rates, while HMC converges an order of magnitude more quickly. The independence sampler does converge, to some extent, to the mean value of these observables despite its rejection rate, in that the method converges to a "good" vector field and then stays there.

More challenging observables to resolve are scalar differences[78, 84],

$$
\Delta_{r} \theta=\theta(\mathbf{x}+\mathbf{r}, t)-\theta(\mathbf{x}, t)
$$

which require resolving $\theta$ at two different locations. Figure 8.8 shows convergence results for the mean values (cumulative moving averages) of scalar differences with $\mathbf{x}=[0,0]$ and $\mathbf{r}_{\mathbf{i}}=2^{-i}[1,1]$ for $i=1,2,3,4$, up to 100,000 samples (10 times longer than the results shown

\footnotetext{
${ }^{2}$ The sharp downward dips in these relative error plots occur when a cumulative moving average drifts past the "true" value. At these points, the relative error is zero, so a log plot exhibits a downward dip because $\log (0)=-\infty$.
} 

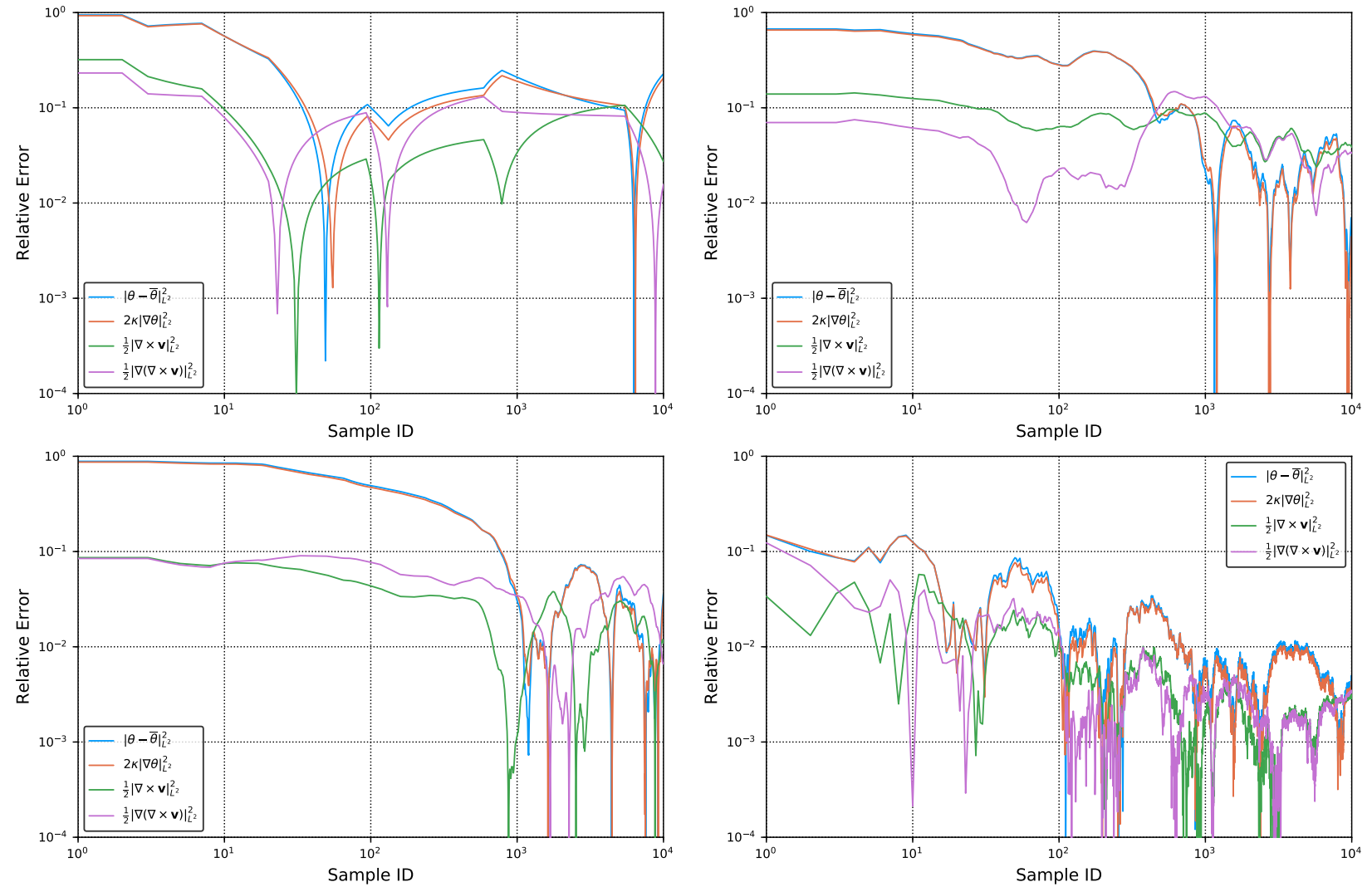

Figure 8.7: Relative error for the mean value (cumulative moving average) of observables scalar variance, scalar dissipation rate, enstrophy, and enstrophy dissipation for 10,000 samples, IS (top left), pCN (top right), MALA (bottom left), and HMC (bottom right). 
in Figure 8.7).

The results show no convergence at all (within the 100,000 sample window) for the independence sampler. pCN and MALA again show noisy but similar convergence. The mean for HMC, by contrast, stabilizes very quickly but then exhibits slow convergence to converge to the "true" value.
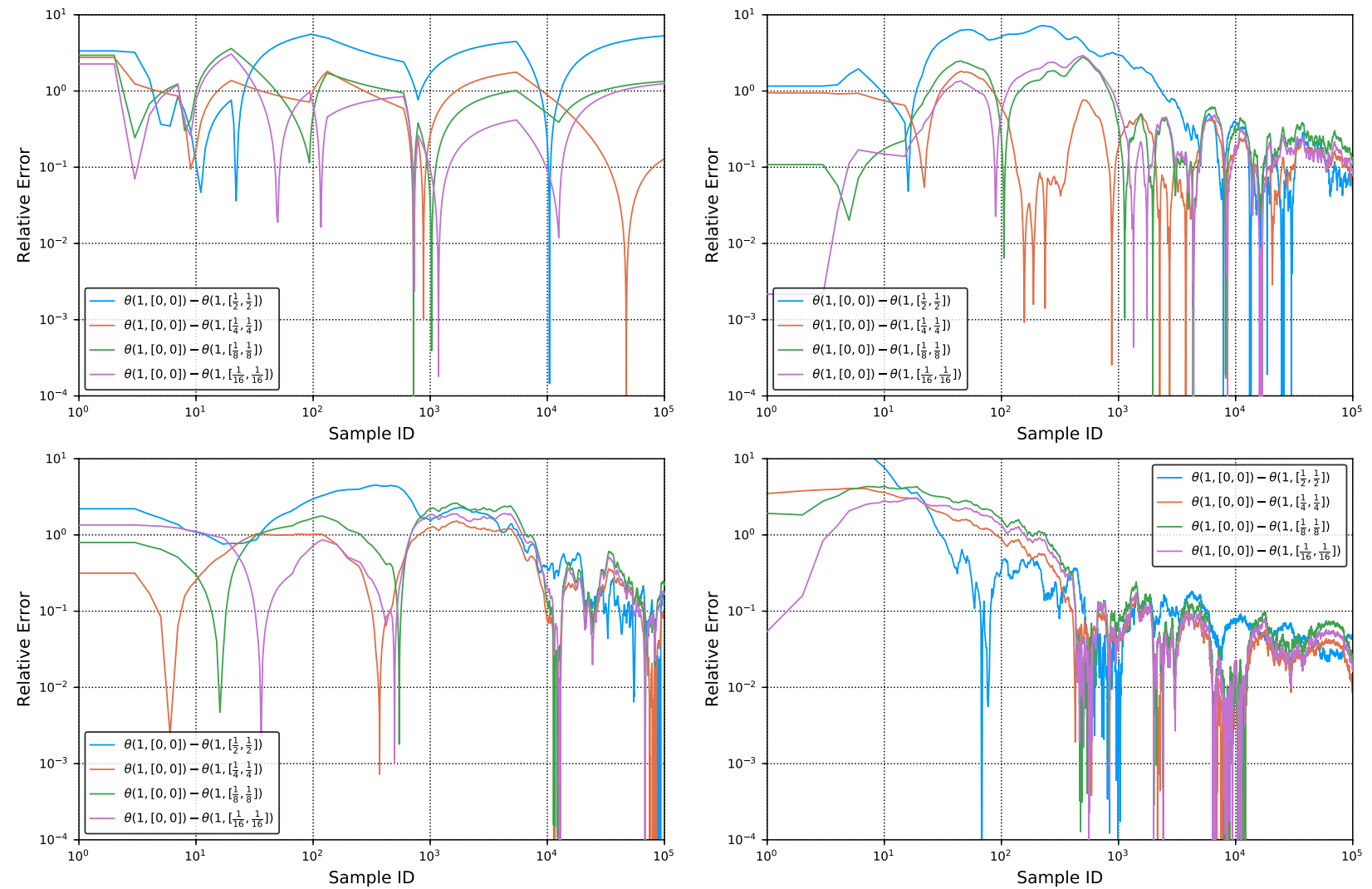

Figure 8.8: Relative error for the mean value (cumulative moving average) of scalar differences at $t=1$ between the origin and $\left[\frac{1}{2}, \frac{1}{2}\right],\left[\frac{1}{4}, \frac{1}{4}\right],\left[\frac{1}{8}, \frac{1}{8}\right],\left[\frac{1}{16}, \frac{1}{16}\right]$ for 100,000 samples, IS (top left), pCN (top right), MALA (bottom left), and HMC (bottom right).

\subsubsection{Convergence of Measures}

We now consider how the computed probability density functions (normalized histograms) compare for each of the MCMC methods. Figure 8.9 shows the computed marginal dis- 
tributions for the first few vector field components after 10,000 samples. This figure can be compared with the "true" marginal distributions shown in Figure 8.4. The difference between these distributions can be computed via the total variation distance

$$
d_{k}\left(\mu, \mu^{(N)}\right)=\frac{1}{2} \int\left|\frac{d \mu}{d \mu_{0}}\left(v_{k}\right)-\frac{d \mu^{(N)}}{d \mu_{0}}\left(v_{k}\right)\right| \mu_{0}\left(d v_{k}\right)
$$

between the "true" $\mu$ and $N$-sample $\mu^{(N)}$ marginal distributions. Convergence of the MCMC chains to the true marginal distributions are shown in Figure 8.10. The figure shows HMC achieves a close approximation to the posterior marginal distributions within a few hundred iterations. Convergence is somewhat faster for $\mathrm{pCN}$ than for MALA despite the absence of a $D \Phi$ computation in the former method. 

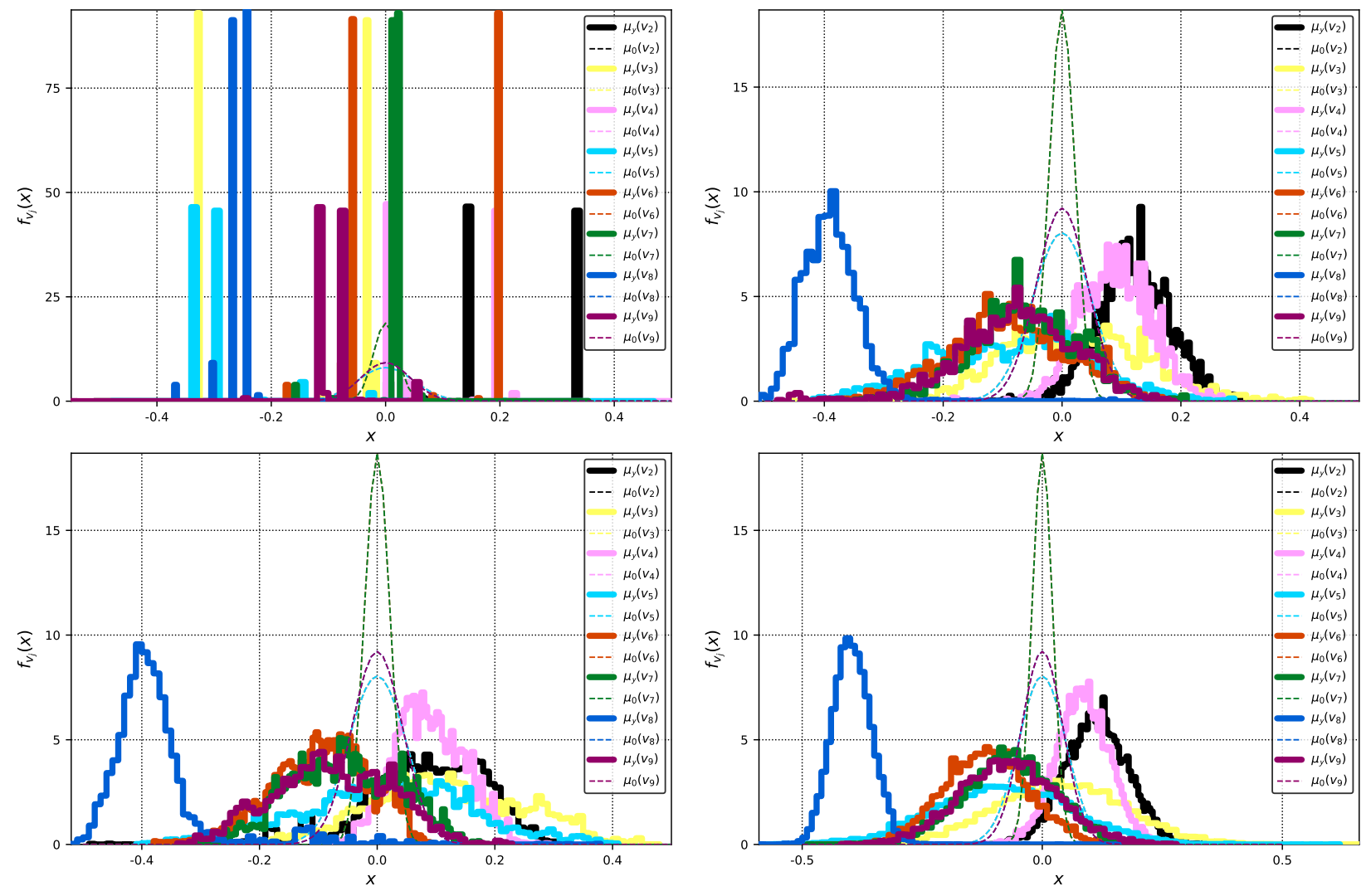

Figure 8.9: Computed marginal distribution for each of the first eight vector field components for 10,000 samples, IS (top left), pCN (top right), MALA (bottom left), and HMC (bottom right). 

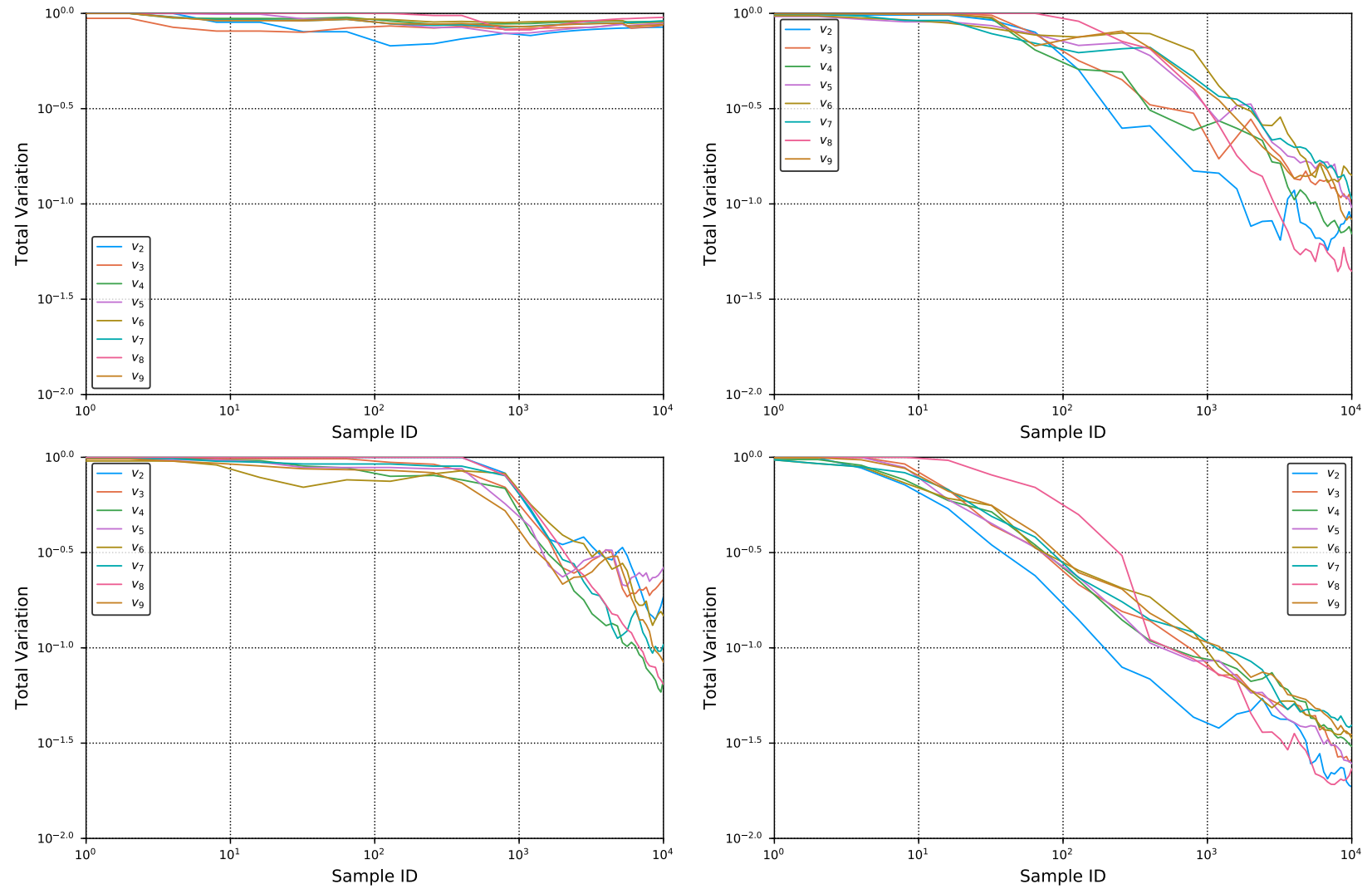

Figure 8.10: Total variation norm between computed and "true" marginal probability density function for $v_{2}, \ldots, v_{9}$ for 10,000 samples, IS (top left), pCN (top right), MALA (bottom left), and HMC (bottom right). 


\subsubsection{Equal Runtime Comparison}

Recall from Table 8.1 that the parameters used for HMC were $\epsilon=0.125$ and $\tau=1.0$, meaning that $\frac{\tau}{\epsilon}=8 \mathrm{PDE}$ and adjoint solves (see Table 5.1) are required per HMC sample. Because of these solves and the additional steps required for the gradient computation (see Algorithm 6) and time integration (see Section 5.5), each HMC sample took the time of approximately 39 IS or pCN samples to compute. Similarly, an HMC sample took an average of 7.7 times as long to generate as a MALA sample, which requires only one PDE and adjoint solve each per sample. Thus, we can reweight IS and pCN samples by 39 and MALA samples by 7.7 to get a comparison of the sampling accuracy per unit time. Figure 8.11 and Figure 8.12 show the convergence of observables and total variation norm for chains of runtime equal to 10,000 samples of HMC; the results can be compared with Figure 8.7 and Figure 8.10, respectively. We see that chains of equal runtime are largely equivalent across methods when applied to Example 1 (except the independence sampler); the faster convergence of HMC is mostly eliminated by the larger amount of computation required to generate the samples. 

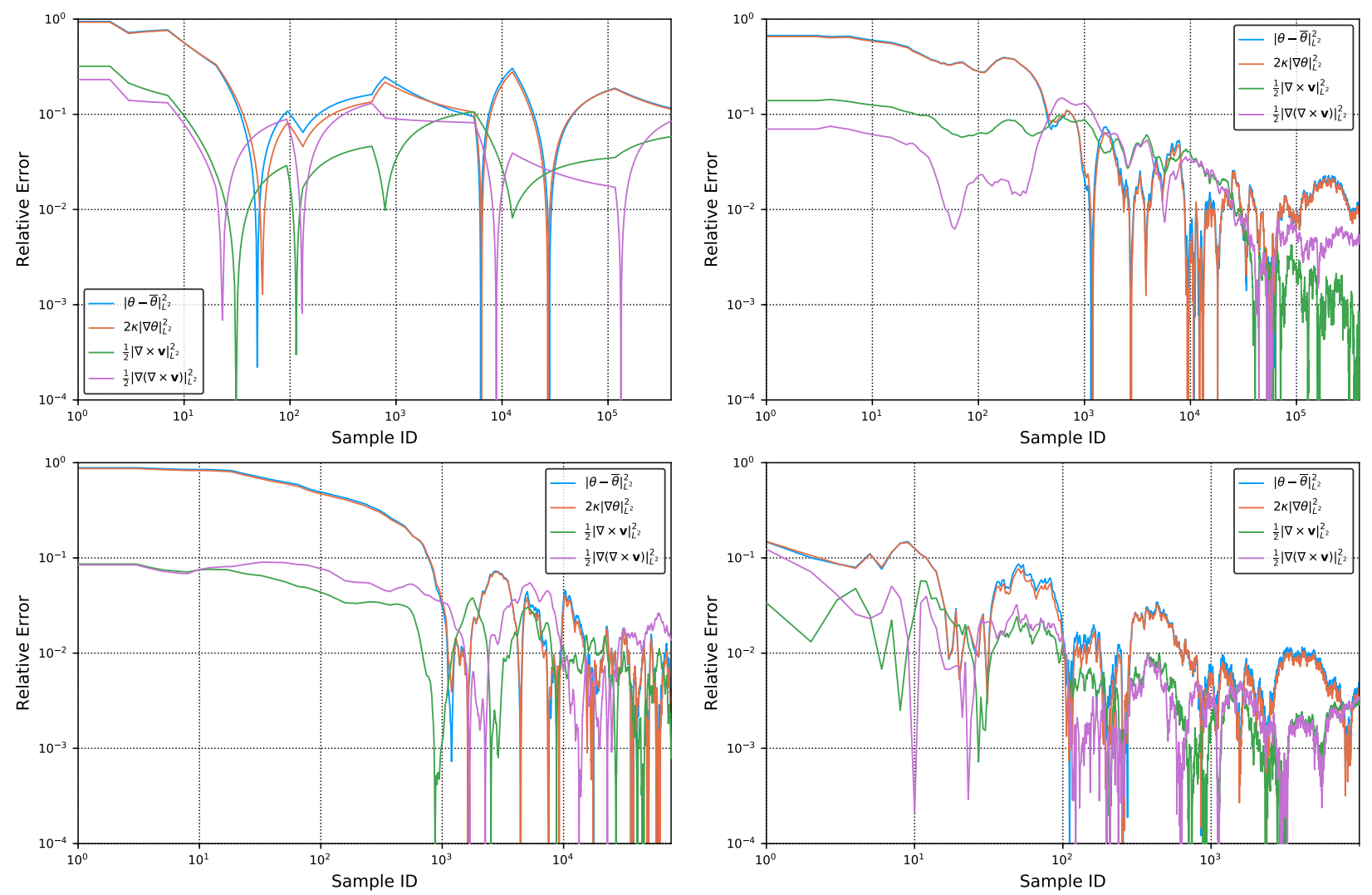

Figure 8.11: Relative error for the mean value (cumulative moving average) of observables scalar variance, scalar dissipation rate, enstrophy, and enstrophy dissipation for runtime equivalent to 10,000 HMC samples, IS (top left), pCN (top right), MALA (bottom left), and HMC (bottom right). 

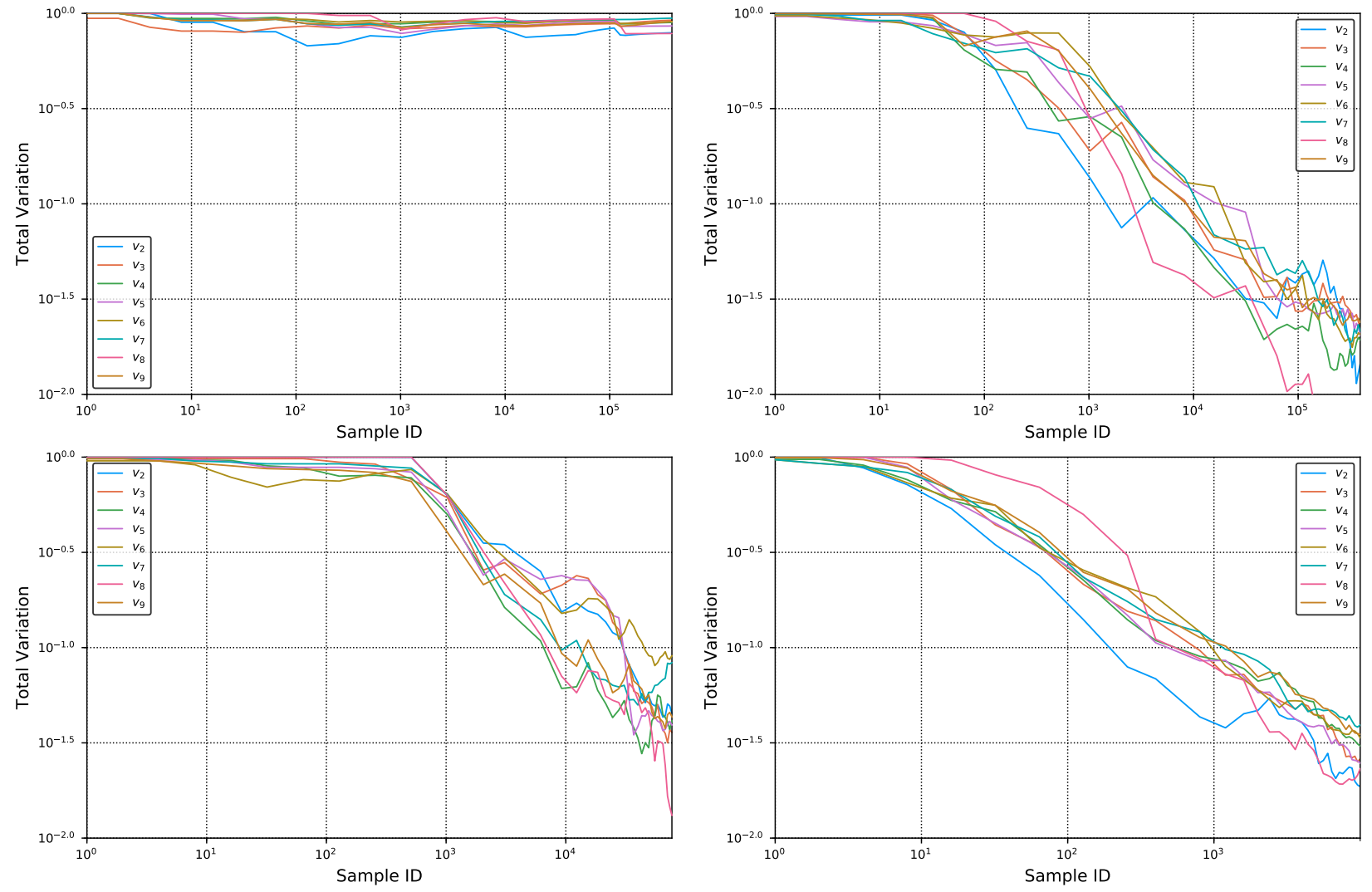

Figure 8.12: Total variation norm between computed and "true" marginal probability density function for $v_{2}, \ldots, v_{9}$ for runtime equivalent to 10,000 HMC samples, IS (top left), pCN (top right), MALA (bottom left), and HMC (bottom right). 


\subsection{Example 2: Multi-welled Posterior}

In this section we present an example where the prior and data interact to produce a more complex posterior. We will see that the example produces a posterior with multiple wells (regions of mass); posteriors of this kind are difficult for MCMC methods to resolve because chains have trouble jumping between the wells. We take the initial condition:

$$
\theta_{0}(\mathbf{x})=\frac{1}{2}-\frac{1}{4} \cos 2 \pi x-\frac{1}{4} \cos 2 \pi y
$$

This initial condition is shown in Figure 8.13.

Let the true vector field be

$$
\mathbf{v}^{\star}=\left[\begin{array}{c}
8 \cos 2 \pi y \\
8 \cos 2 \pi x
\end{array}\right]
$$

This vector field is shown in Figure 8.14. Note that only two Fourier components of $\mathbf{v}^{\star}$ are non-zero.

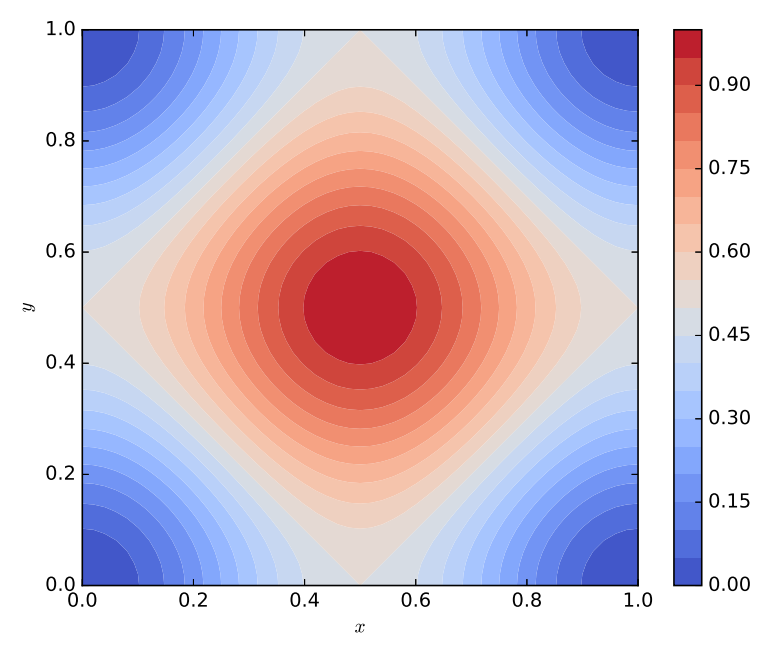

Figure 8.13: $\theta_{0}(\mathbf{x})$ for Example 2.

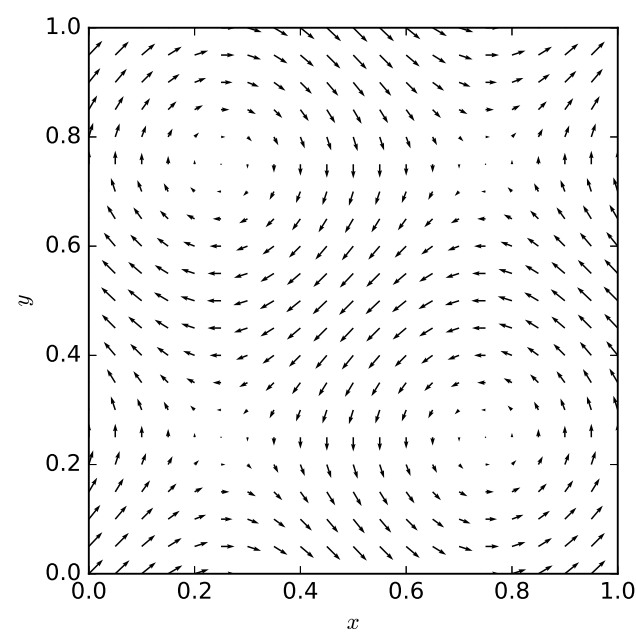

Figure 8.14: $\mathbf{v}^{\star}$ for Example 2 . 
Symmetry guarantees that for $\mathbf{x}_{1}=[0,0]$ and $\mathbf{x}_{2}=\left[\frac{1}{2}, \frac{1}{2}\right]$ we have

$$
\begin{aligned}
& \theta\left(\mathbf{v}^{\star}, t, \mathbf{x}_{1}\right)=\theta\left(-\mathbf{v}^{\star}, t, \mathbf{x}_{1}\right) \\
& \theta\left(\mathbf{v}^{\star}, t, \mathbf{x}_{2}\right)=\theta\left(-\mathbf{v}^{\star}, t, \mathbf{x}_{2}\right)
\end{aligned}
$$

(In fact, there are more points for which this is true; however, two points suffice for the purposes of this example.)

Let the data $\mathcal{Y}$ be point measurements $\theta(t, \mathbf{x})$ from $t=0.001$ to 0.050 in intervals of 0.001 at each of $\mathbf{x}_{1}$ and $\mathbf{x}_{2}$. Then by (8.8), we have $\Phi\left(\mathbf{v}^{\star}\right)=\Phi\left(-\mathbf{v}^{\star}\right)$, i.e. both $\mathbf{v}^{\star}$ and $-\mathbf{v}^{\star}$ match the data equally well.

Finally, we use the mean-zero Kraichnan prior described in Section 8.1, which assigns both $\mathbf{v}^{\star}$ and $-\mathbf{v}^{\star}$ the same probability according to the prior. Since both $\mathbf{v}^{\star}$ and $-\mathbf{v}^{\star}$ are given the same probability by both the prior and the data, each will be equally likely according to the posterior - that is, we expect the posterior distribution to have multiple wells as long as our observations are sufficiently precise to "draw" the single-welled prior toward vector fields that match the data.

The problem parameters for this example are listed in Table 8.5. We will see that the symmetry of the problem setup results in multiple distinct probability masses in the posterior; this structure can be difficult for MCMC methods to resolve efficiently, as the chains tend to get stuck in one of the wells and have trouble jumping to the others. We will explore this behavior in detail in Section 8.3.3. 


\begin{tabular}{|l|l|l|}
\hline Parameter & Symbol & Value \\
\hline \hline Observation operator & $\mathcal{O}$ & Point observations at $\mathbf{x}_{1}=[0,0]$ and $\mathbf{x}_{2}=\left[\frac{1}{2}, \frac{1}{2}\right]$ \\
\hline Prior distribution & $\mu_{0}$ & Kraichnan (see Section 8.1$)$ \\
\hline Observed data & $\mathcal{Y}$ & $\mathcal{G}\left(\mathbf{v}^{\star}\right)$ for some $\mathbf{v}^{\star}$ \\
\hline True vector field & $\mathbf{v}^{\star}$ & {$[8 \cos 2 \pi y, 8 \cos 2 \pi x]$} \\
\hline Diffusion parameter & $\kappa$ & $3 \times 10^{-5}$ Table I, $[18]$ \\
\hline $\begin{array}{l}\text { Observational noise } \\
\text { distribution }\end{array}$ & $\mathbb{Q}_{0}$ & $N\left(0, \sigma_{\eta} I\right), \sigma_{\eta}=2^{-3}$ \\
\hline Initial condition & $\theta_{0}$ & $\frac{1}{2}-\frac{1}{4} \cos (2 \pi x)-\frac{1}{4} \cos (2 \pi y)$ \\
\hline
\end{tabular}

Table 8.5: Problem parameters for Example 2.

\subsubsection{Posterior Structure}

As in Example 1, we approximate the exact posterior via a large number of pCN samples; in this case 25 million samples were generated from 100 pCN chains of 250,000 samples each, each beginning with a randomly-chosen initial sample. (For a discussion of why multiple chains were used, see Section 8.3.) Figure 8.15 presents the resulting marginal distributions for the first few components of $\mathbf{v}$ (see (8.4) for interpretation of the components). Note that the symmetry of the problem results in multiple large humps both $v_{2}$ and $v_{4}$, as well as in several smaller bumps in the distributions of the other components.

Moreover, unlike in Example 2, these vector field components are highly correlated with each other, even though the Kraichnan prior assumes independence of the components. Twodimensional histograms representing the approximate posterior joint probability density of pairs of vector field components are shown in Figure 8.16 (compare with Figure 8.5 for Example 1). The posterior shows a number of complicated structures, such as the Star Wars "TIE Fighter" [85] between $v_{2}$ and $v_{3}$, the "baseball diamond" shape[1] between $v_{2}$ and $v_{4}$, the "X" shape between $v_{3}$ and $v_{8}$, and the circular structure between, e.g., $v_{3}$ and $v_{9}$. 


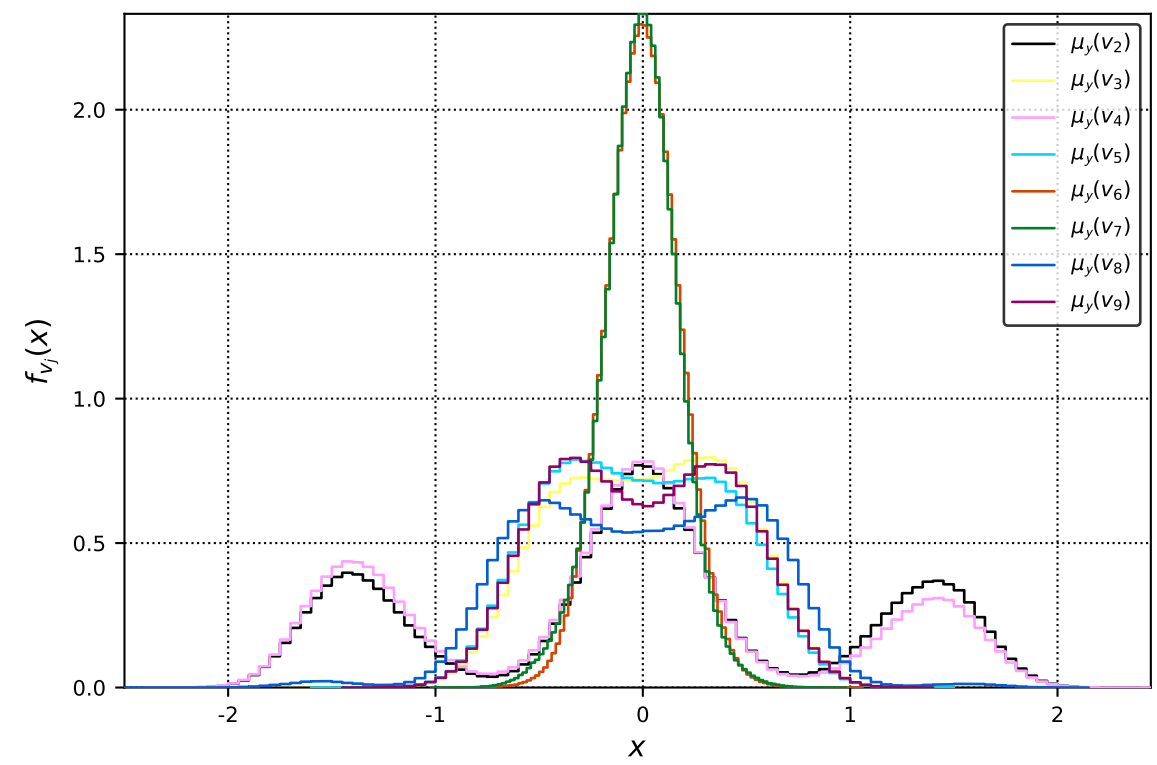

Figure 8.15: Posterior $\left(\mu_{y}\right)$ marginal distributions for the first eight components of $\mathbf{v}$.

It is worth noting that the posterior contains these correlation structures even though the Kraichnan prior assumes independence of the components.

Finally, it is worth noting that not all observables of the posterior will exhibit complicated structures. In fact, the symmetry of the problem guarantees that some will not. Figure 8.17 shows the computed posterior probability density function for one such example, vector field enstrophy. Since enstrophy is the same for $\mathbf{v}$ and $-\mathbf{v}$, this distribution contains a single mass of probability.

\subsubsection{Sampling and Selection of Free Parameters}

As described in Chapter 5, several of the MCMC methods have free parameters that can be tuned to the problem. In this section, we discuss the effect of these parameters and how we chose their values for Example 2. The plots below were generated by running a number of short examples with various parameter values; longer runs for selected values matched very 


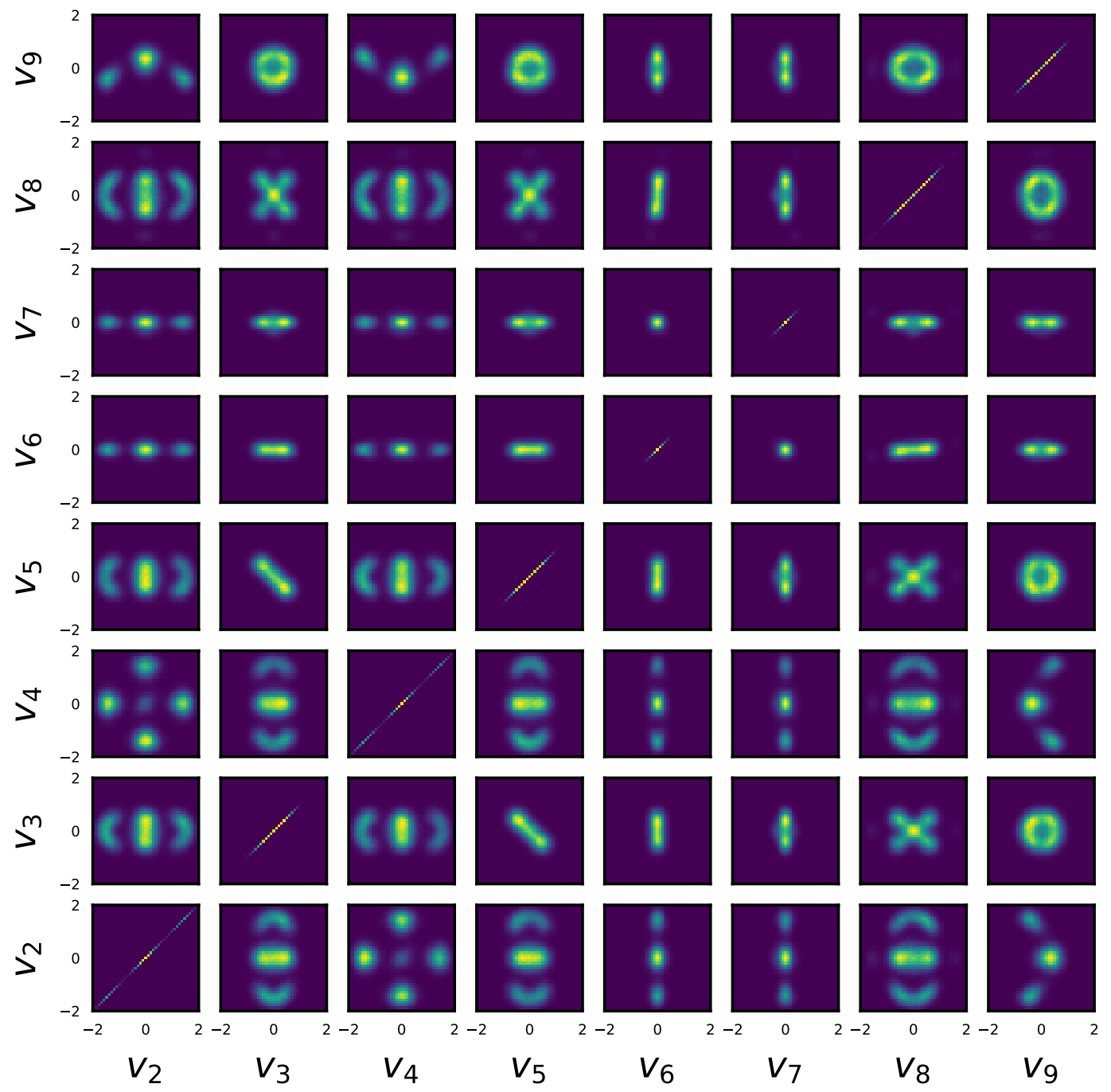

Figure 8.16: Posterior two-dimensional histograms for the first eight components of $\mathbf{v}$. 


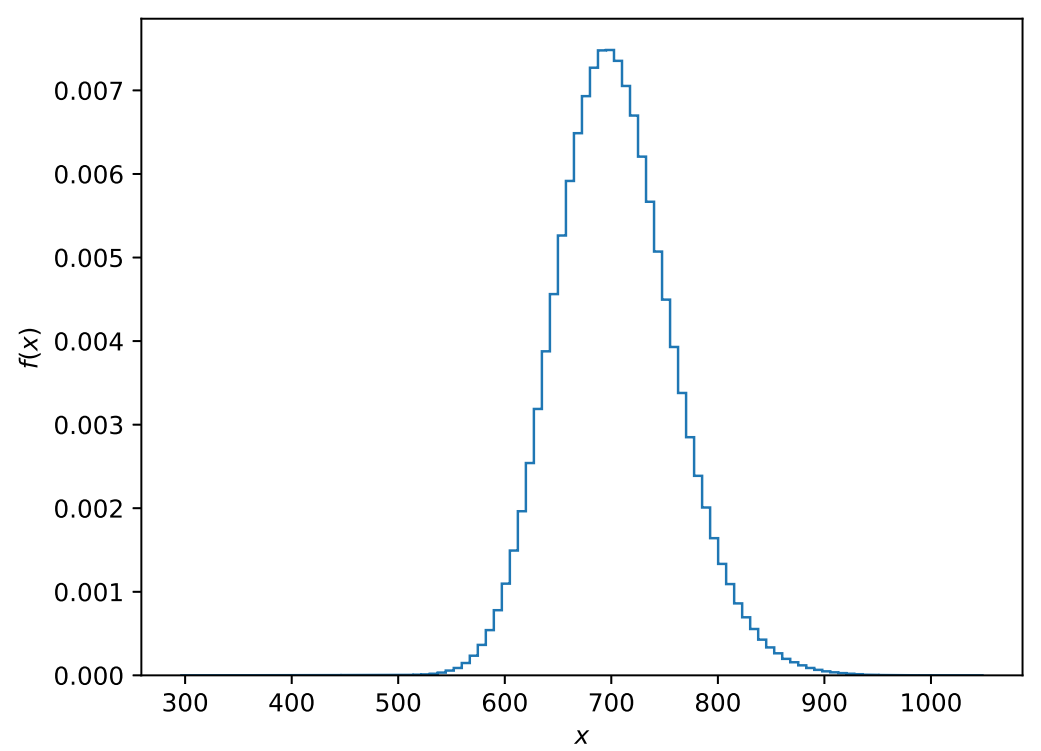

Figure 8.17: Posterior probability density function for vector field enstrophy $\left(\frac{1}{2}\|\nabla \times \mathbf{v}\|_{L^{2}}^{2}\right)$.

well the acceptance rates generated by the shorter runs.

Recall from Section 5.3 that the pCN algorithm includes a free parameter $\beta \in(0,1]$, which determines how aggressive to be in jumping from one sample to the following proposal. The effect of $\beta$ on the pCN acceptance rate for Example 2 is shown in Figure 8.18. Note that the acceptance rate rapidly decreases as $\beta$ increases. $(\beta=1$ corresponds to the Independence Sampler, where proposals are entirely independent of the preceding sample.) Acceptance rates of $\approx 23 \%$ have been shown to be optimal for some classes of high-dimensional MCMC algorithms [71], which for Example 2 corresponds roughly to $\beta=0.2$. This is the value that we selected for the following section.

As described in Section 5.4, the MALA algorithm has a parameter $h>0$, which is the step size used in the discretization of the Langevin SDE. Larger values allow larger jumps between the sample and subsequent proposal, but potentially also increase discretization error and therefore increase rejections (which would be 0 if we could sample from the Langevin equation 
without discretizing). Figure 8.19 shows the computed acceptance ratio as a function of $h$ for the MALA algorithm applied to Example 2, and show rapid deteroriation as $h$ increases. We selected $h=0.01$, which corresponds roughly to the optimal acceptance rate of 0.57 from $[71]$

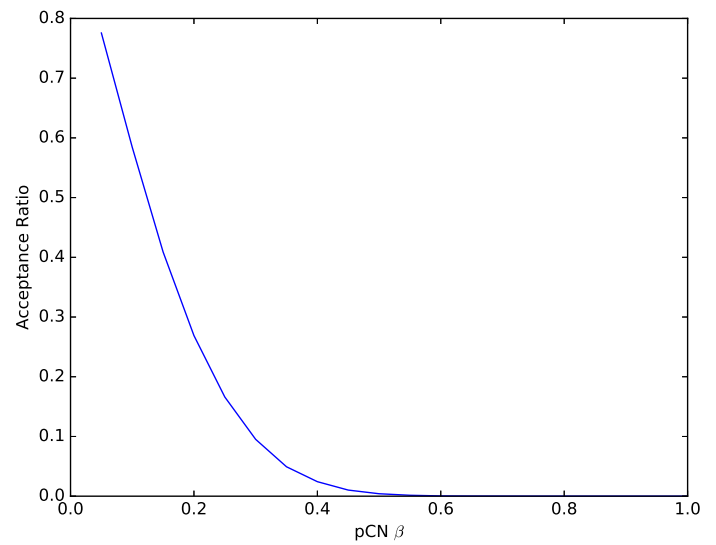

Figure 8.18: Acceptance ratio by $\mathrm{pCN} \beta$.

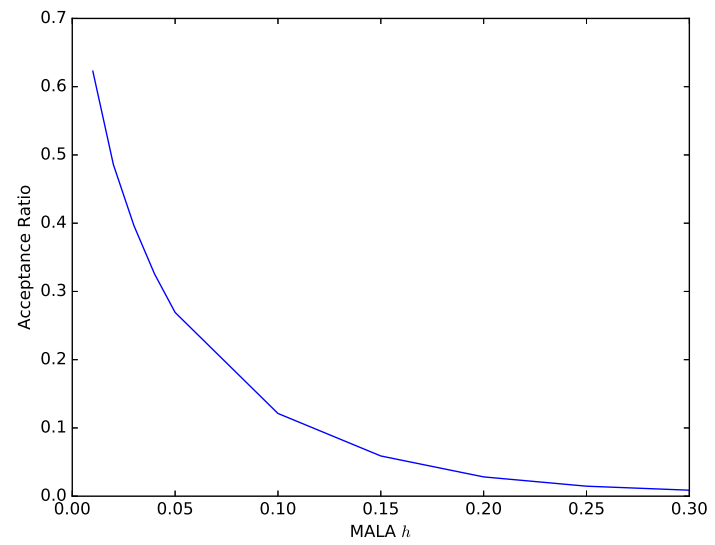

Figure 8.19: Acceptance ratio by MALA $h$.

The HMC algorithm, discussed in Section 5.5, includes two parameters, $\epsilon$ and $\tau$. $\epsilon>0$ is a discretization step size, similar to MALA's $h . \tau>0$ is the integration end time; larger values increase the size of potential jumps between samples but also the computational cost of generating each sample. (Note that one of these two variables could also be replaced with $I:=\frac{\tau}{\epsilon}$, the number of integration steps required per sample.) The acceptance ratio as a function of $\tau$ and $I$ is shown in Figure 8.20. We note that the acceptance rate depends very weakly on $\tau$; for example, $\tau=1, I=8$ and $\tau=0.5, I=4$ (both $\epsilon=0.125$ ) have nearly the same acceptance rate. In practice, we decided how far we wanted to integrate (e.g., to $\tau=1$ ) and then chosen the value of $\epsilon$ that gave a reasonable acceptance rate.

Finally, we note that, in contrast to the other algorithms, the HMC parameters have considerable effect not only on acceptance rate but also on the computational cost required to generate each sample. MALA is much more costly than $\mathrm{pCN}$, requiring an adjoint solve 
per sample and a series of integrations to evaluate the gradient of the potential $\Phi$ (see Section 6.2). HMC for $I=1$ is similar cost to MALA; however, for $I=2$ samples take twice as long to generate, $I=4$ four times as long, and so on. The sampling rate (samples per unit time, normalized so that the Independence Sampler rate is 1) is shown in Figure 8.21.

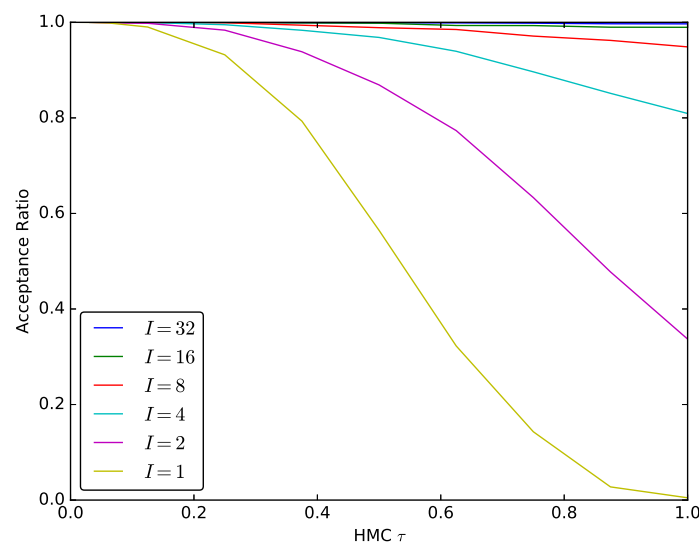

Figure 8.20: Acceptance ratio by HMC $\tau$ and $I=\frac{\tau}{\epsilon}$.

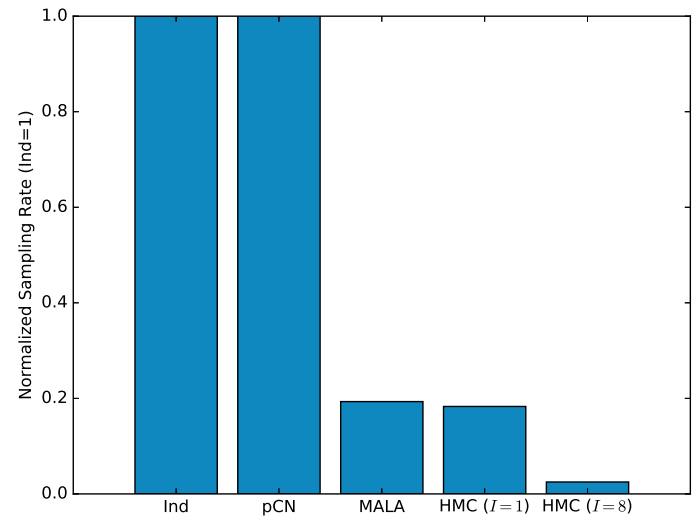

Figure 8.21: Normalized sampling rate by MCMC method (Higher is better, Independence Sampler $=1$ ).

Parameters applied to Example 2 are summarized in Table 8.2. As in Example 1, when the Independence Sampler is applied to Example 2 the acceptance ratio is too low to properly explore the posterior. We therefore omit it from further analysis in this section.

\begin{tabular}{|c|c|c|}
\hline Method & Parameter & Value \\
\hline \hline pCN & $\beta$ & 0.200 \\
\hline MALA & $h$ & 0.001 \\
\hline HMC & $\epsilon$ & 0.125 \\
\hline HMC & $\tau$ & 4.000 \\
\hline
\end{tabular}

Table 8.6: MCMC Parameters Applied to Example 1.

As described earlier, the multi-hump structure of the posterior for Example 2 is typical of distributions that are difficult for MCMC methods to resolve efficiently, as the chains have 
difficulty moving across the regions of low probability between the regions of mass. We will use this structure to test the viability of each MCMC method in resolving complicated posteriors. We will emphasize pCN and HMC, which represent the "cheap" and "expensive" ends of the computational spectrum, though we will show some results for MALA as well.

Figure 8.22 shows the trace and autocorrelation of the potential $\Phi$ (see (3.27)) for MCMC sampling of Example 2. As in Example 1, we see "random walk" behavior for both pCN and MALA algorithms, whereas for HMC many fewer iterations are required to achieve statistical independence between samples. Unlike Example 1, however, the HMC chains for Example 2 exhibit negative autocorrelation between consecutive samples. The author plans to investigate this phenomenon, which may be related to the multi-hump structure of the problem, in later work.
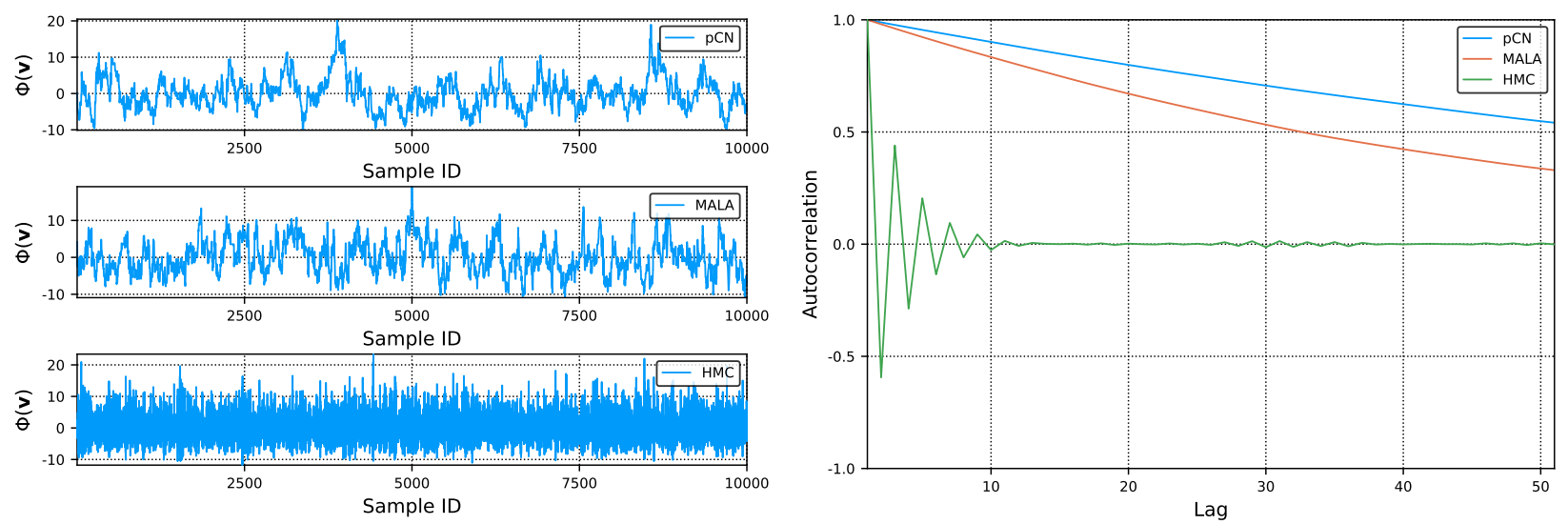

Figure 8.22: Trace (left) and autocorrelation (right) of the potential $\Phi$.

We can also see the contrast between $\mathrm{pCN}$ and $\mathrm{HMC}$ in the traces of vector field components shown in Figure 8.23 and Figure 8.24, respectively. pCN samples move within a relatively limited range (a single probability mass), while the HMC samples occasionally jump between the different probability regions. In parameter testing, we observed that the frequency of these jumps increased roughly linearly with $\tau: \tau=4$ produced twice as many jumps as $\tau=2$, for example - because longer integration times allowed the Hamiltonian system to 
evolve further, overcoming the areas of low probability separating the humps.

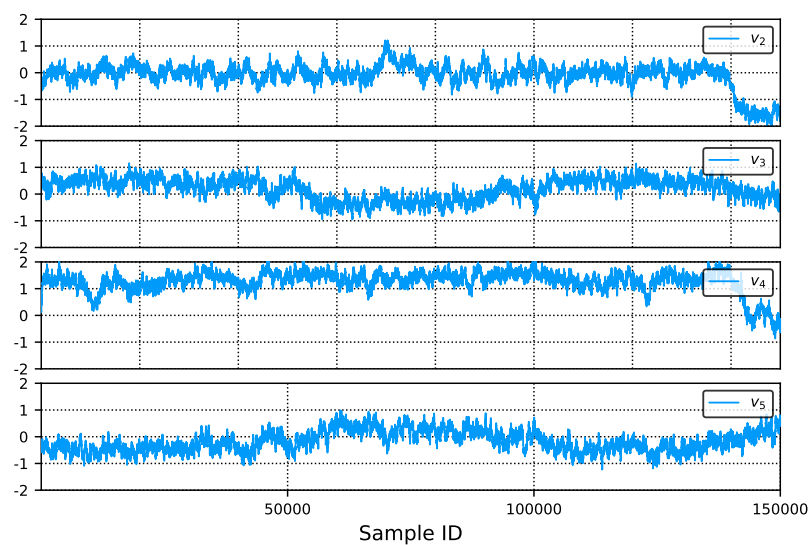

Figure 8.23: Trace of $v_{2}, \ldots, v_{5}$ by sample number, $\mathrm{pCN}$.

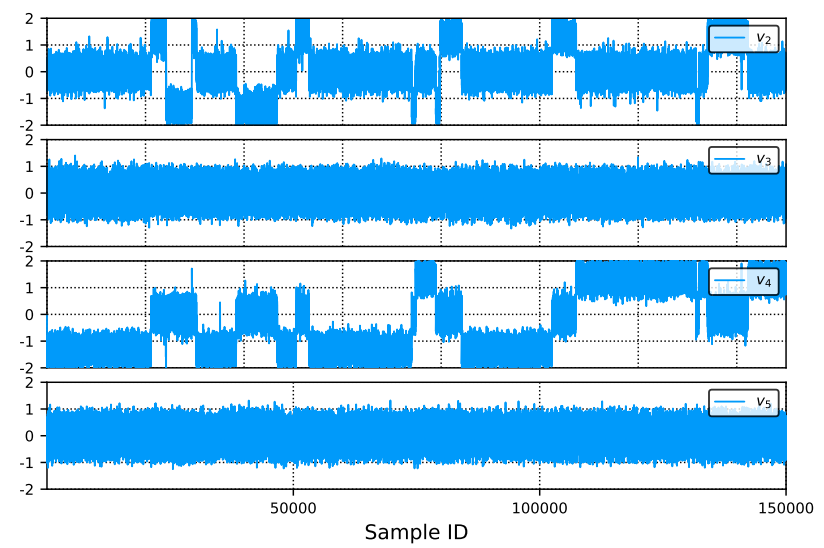

Figure 8.24: Trace of $v_{2}, \ldots, v_{5}$ by sample number, HMC.

\subsubsection{Convergence of Observables}

As in Example 1, we compare convergence of several observables relevant to the passive scalar community, summarized in Table 8.4. The posterior samples described in Section 8.3.1 are used as the "true" values for comparison. Figure 8.25 shows the cumulative moving average of scalar variance, scalar dissipation rate, enstrophy, and enstrophy dissipation for pCN and HMC. The means for both methods converge, though HMC converges in an order of magnitude fewer samples than pCN.

Figure 8.26 shows similar convergence plots for scalar differences, which proved much harder for the MCMC methods to resolve; the figure shows results through 150,000 samples (15 times more samples than in Figure 8.25). pCN fails to converge for two of the values; this is due to the fact that the pCN chain fails to resolve all regions of the multi-hump distribution. HMC does eventually start to converge to the true value. The sawtooth shape of the mean values are reflective of the jumping between humps seen in Figure 8.24; convergence in this case involves balancing the number of samples that the chain produces in each of the various 
probability humps.
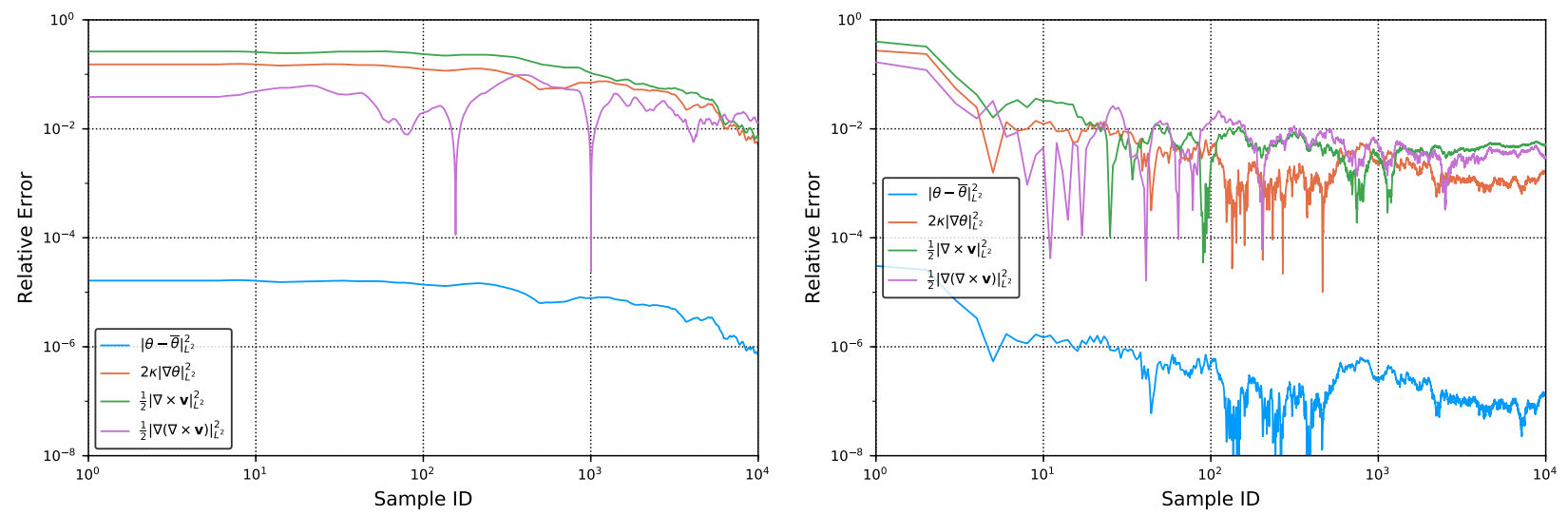

Figure 8.25: Relative error for the mean value of observables scalar variance, scalar dissipation rate, enstrophy, and enstrophy dissipation for 10,000 samples, pCN (left) and HMC (right).
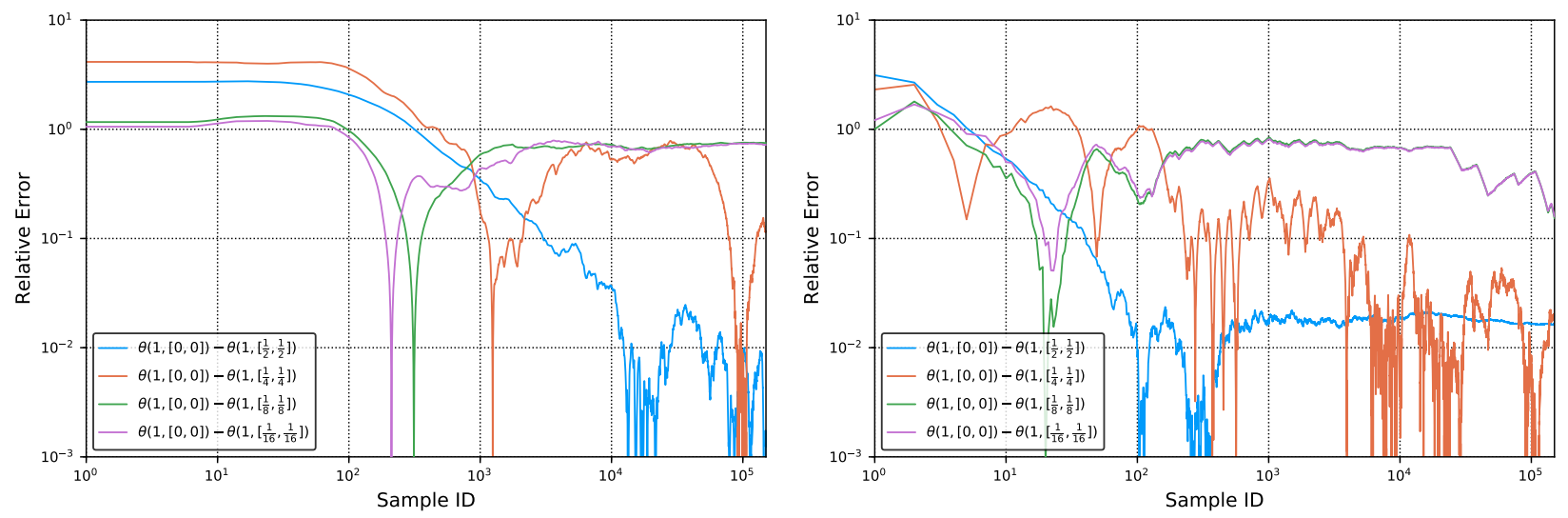

Figure 8.26: Relative error for scalar differences between various points for 150,000 samples, pCN (left) and HMC (right).

\subsubsection{Convergence of Measures}

We now consider how the computed probability density functions (normalized histograms) compare for each of the MCMC methods. Figure 8.27 shows the computed marginal distributions for the first few vector field components after 150,000 samples. This figure can be compared with the "true" marginal distributions shown in Figure 8.15. The pCN chain 
appears to have resolved only some portions of the distribution. Many of the computed marginal distributions are asymmetric; the distributions for $v_{2}$ and $v_{4}$, in particular, only show one or two of the three humps shown in Figure 8.15. By contrast, the marginal distributions for $\mathrm{HMC}$ are more symmetric about the origin, and $\mathrm{HMC}$ has been able to resolve the multiple probability humps that the pCN method could not. (The asymmetry in $v_{4}$ and $v_{9}$ indicates that the chain has perhaps not fully converged yet.)

As in Example 1, we can compute the difference between these distributions via the total variation distance (8.6) between the "true" $\mu$ and $N$-sample $\mu^{(N)}$ marginal distributions. Convergence of the MCMC chains to the true marginal distributions are shown in Figure 8.28. As in Figure 8.26, we see a sawtooth behavior for HMC, as the number of samples in each hump of, for example, $v_{4}$ slowly balances.
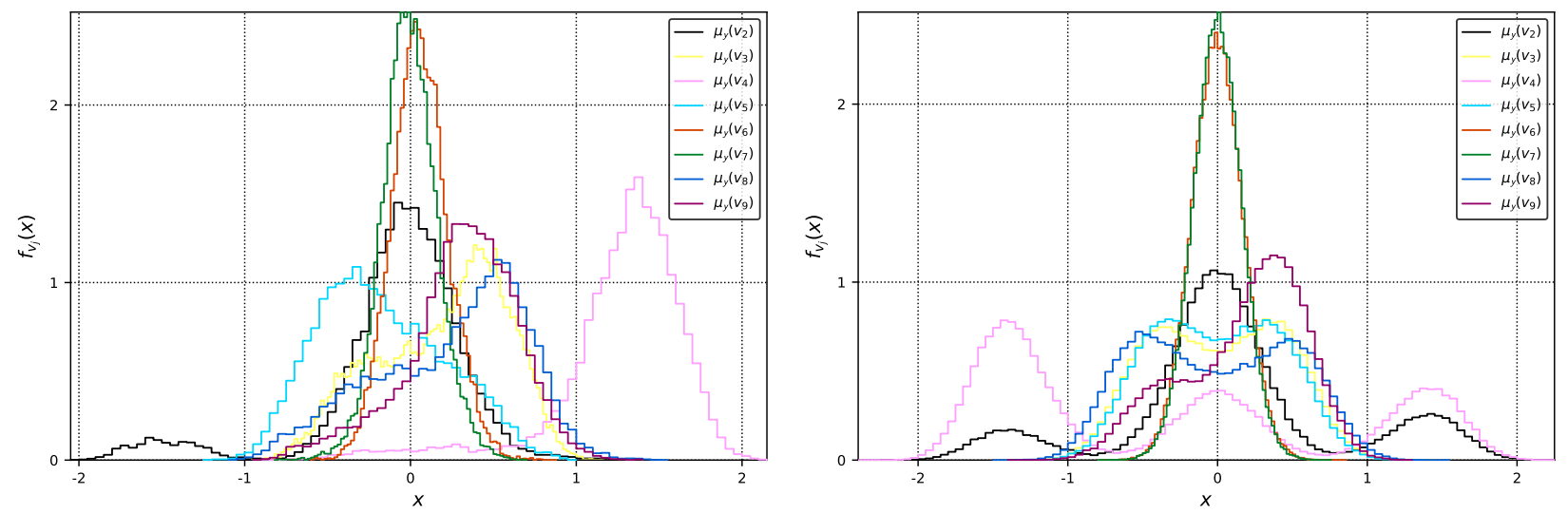

Figure 8.27: Computed marginal distribution for each of the first eight vector field components for 150,000 samples, pCN (left) and HMC (right).

Finally, we present for comparison two-dimensional histograms for the pCN and HMC chains in Figure 8.29 and Figure 8.30, respectively. These can be compared with the "true" histograms in Figure 8.16. The histograms for pCN show only some of the many distinct probability regions in the posterior, as the chain failed to jump across the regions of low probability. The histograms for HMC, by contrast, resolve all major features in the poste- 

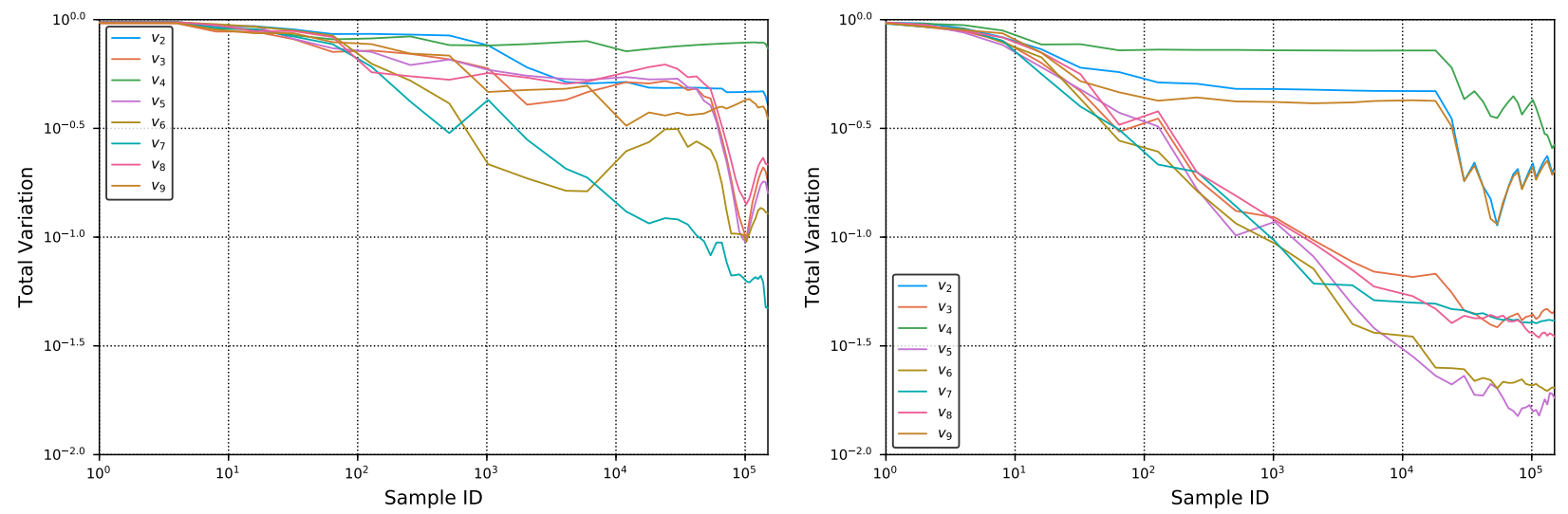

Figure 8.28: Total variation norm between computed (150,000 samples) and "true" marginal probability density function for $v_{2}, \ldots, v_{9}$, pCN (left) and HMC (right).

rior, though the some of the features still exhibited imbalance when the chain terminated. For example, each of the four the "bases" in the $v_{2} / v_{4}$ "baseball diamond" should have equal weight, while HMC gives the $v_{4} \approx-2$ base more weight. This imbalance would probably improve for longer runtimes, as the chain spends equal time in all probability humps. 

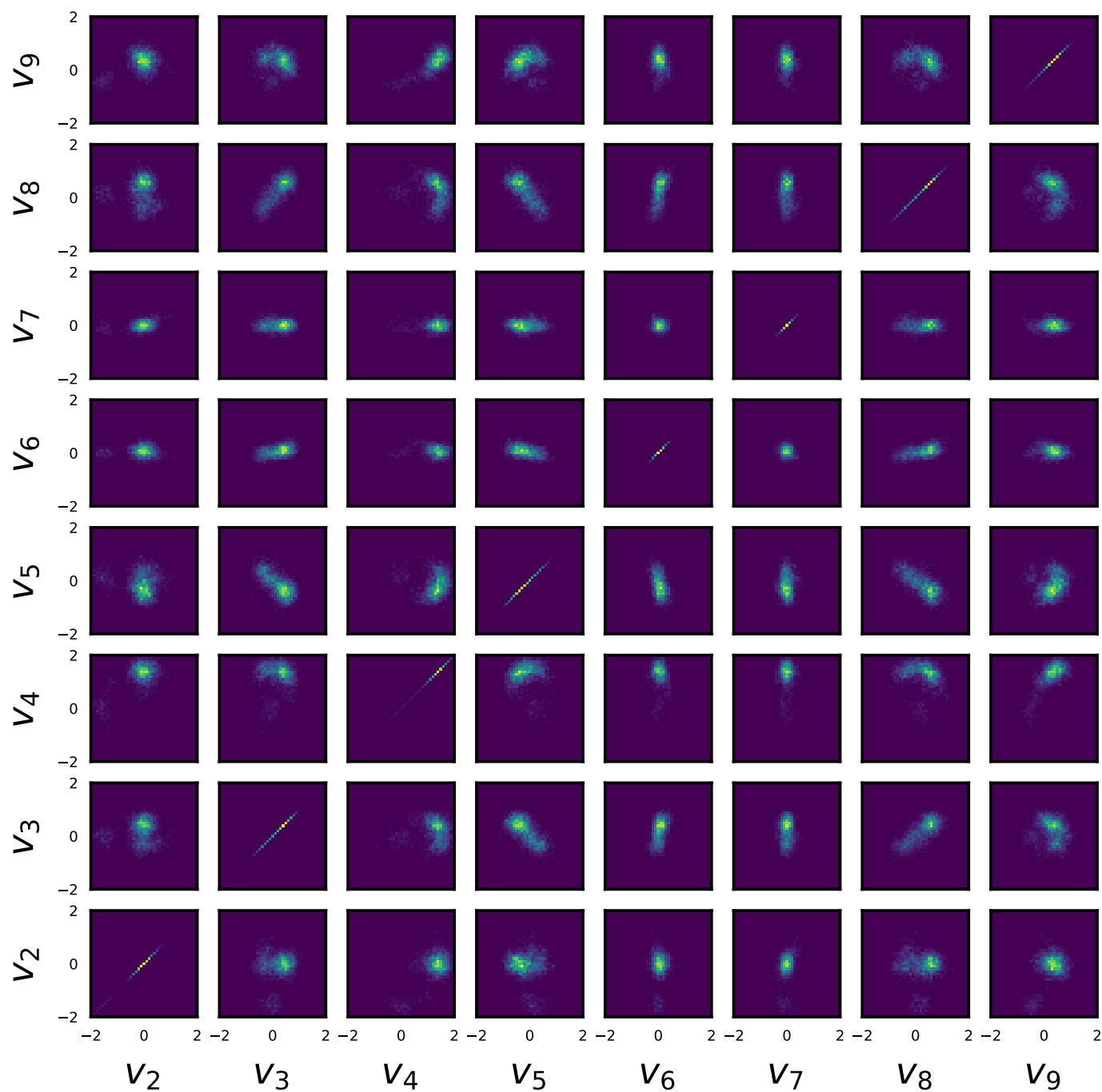

Figure 8.29: Posterior two-dimensional histograms for the first eight components of $\mathbf{v}, \mathrm{pCN}$ sampler (Single chain). 

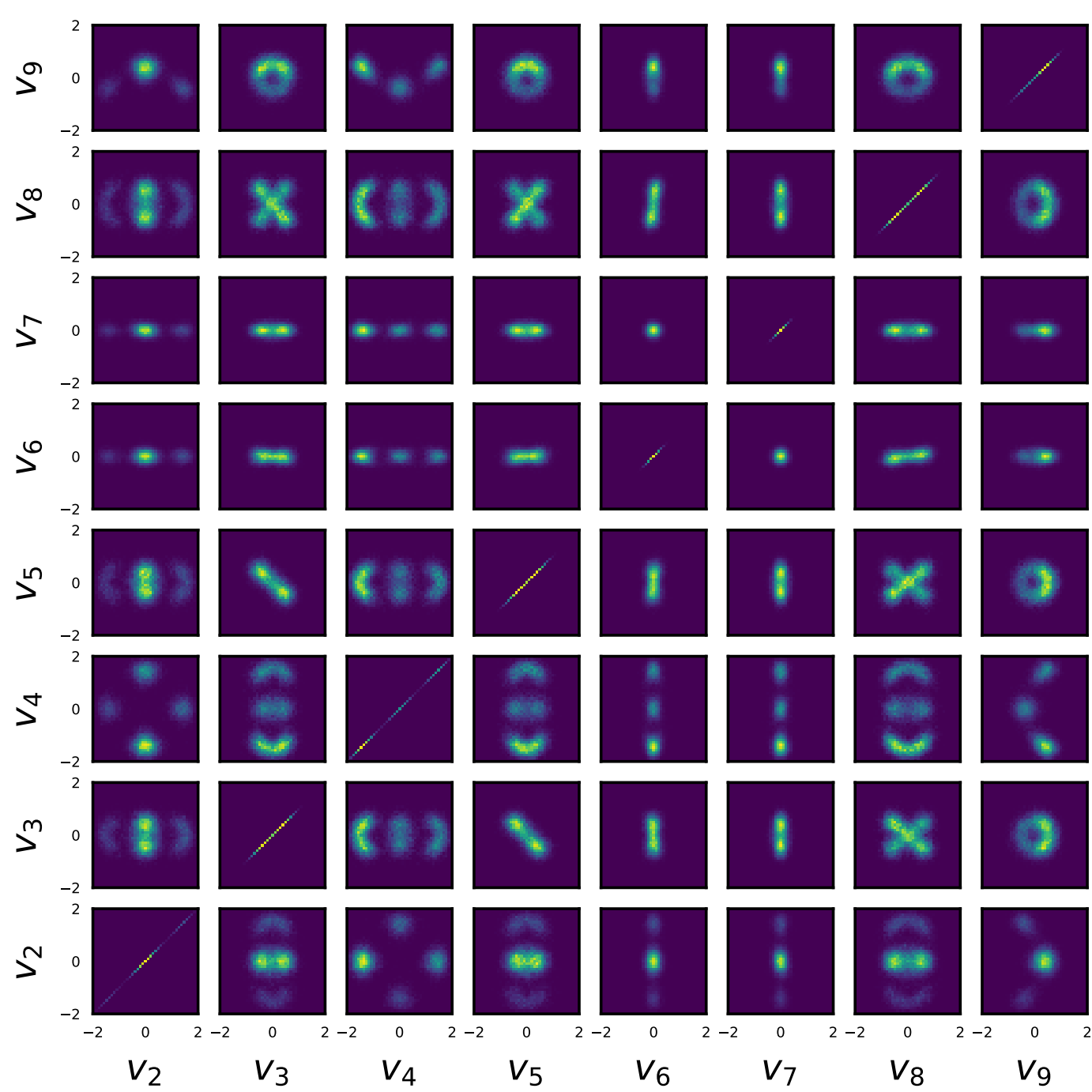

Figure 8.30: Posterior two-dimensional histograms for the first eight components of $\mathbf{v}$, HMC sampler (Single chain). 


\subsubsection{Equal Runtime Comparison}

We note, however, that the flavor of HMC that we used above required 32 PDE and 32 adjoint solves per sample, making it quite expensive relative to pCN. In our implementation, we were able to compute 125 pCN samples for each HMC sample. As a result, almost 19 million pCN samples could be computed in the time required to generate $150,000 \mathrm{HMC}$ samples. For an equal comparison in terms of computational cost, we now present the results of a single 19 million-sample pCN chain with the 150,000 sample HMC run shown earlier. Figure 8.31 compares the trace of the first few vector field components by sample number. We see that pCN does eventually achieve the jumps between states that HMC shows; however, the jumps are much less frequent for $\mathrm{pCN}$, even when weighted by run-time, than for HMC. As a result, the times spent in the various regions are less balanced for pCN than for HMC. Figure 8.32 compares the evolution of the total variation norm between the computed and "true" marginal distributions for $\mathrm{pCN}$ and $\mathrm{HMC}$ chains of equal runtime. We observe that the marginals appear to be converging for both $\mathrm{pCN}$ and HMC at roughly equal rates.
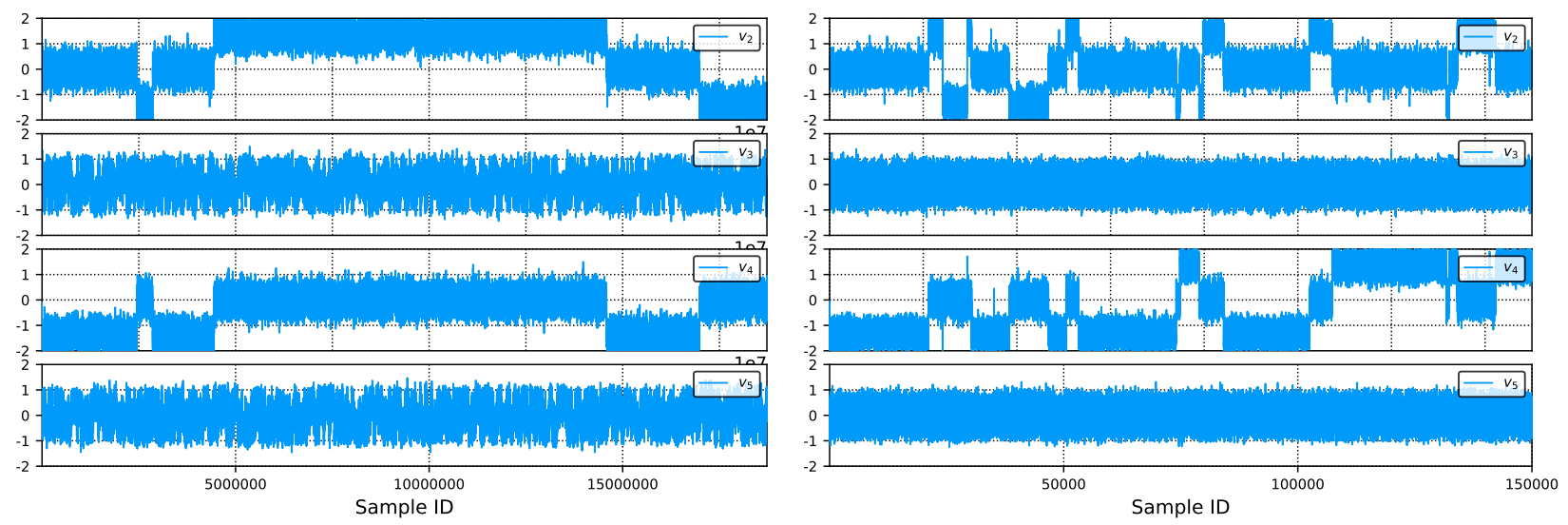

Figure 8.31: Trace of $v_{2}, \ldots, v_{5}$ by sample number for runtime equivalent to 150,000 HMC samples, pCN (left) and HMC (right).

Alternatively, we can split the 19 million pCN samples across several different chains and then assemble the results into a single aggregated list. For example, for 100 pCN chains, 


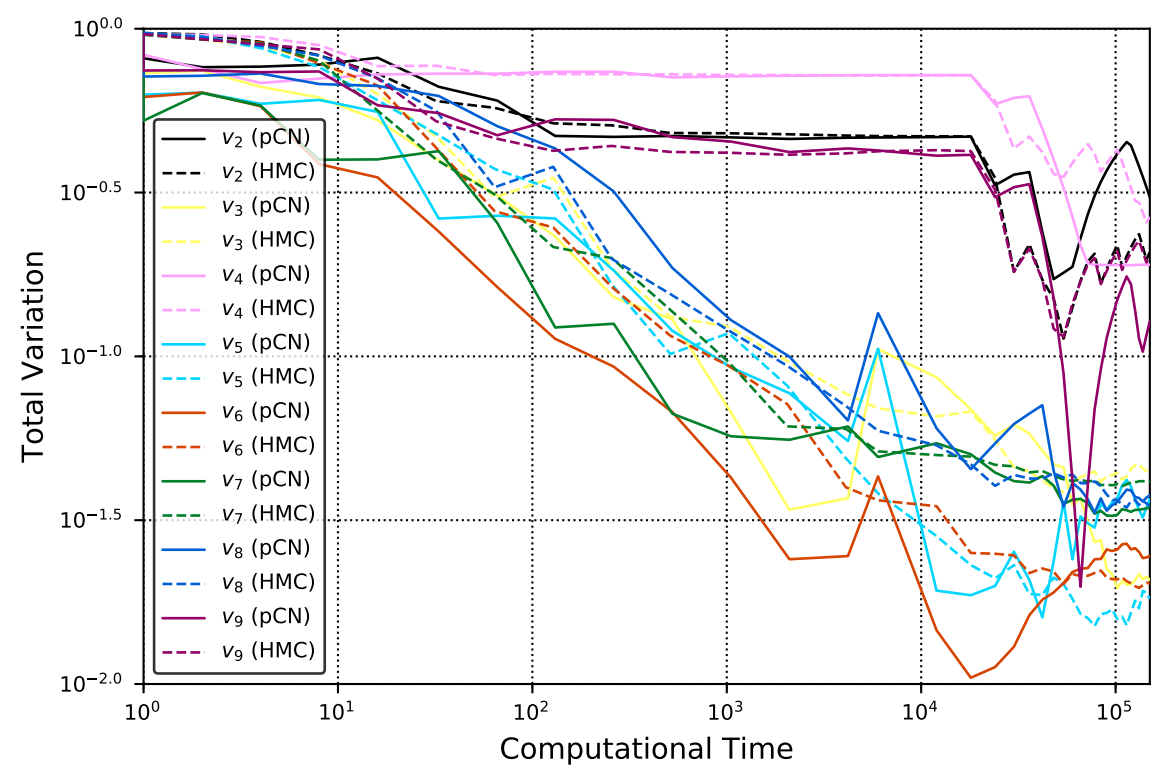

Figure 8.32: Total variation norm between computed and "true" marginal probability density function for $v_{2}, \ldots, v_{9}$ for $\mathrm{pCN}$ and $\mathrm{HMC}$, by computational time (1 computational unit = 1 HMC sample).

Sample 1 of the aggregate would be Sample 1 from Chain 1, Sample 2 of the aggregate would be Sample 1 from Chain 2, Sample 100 of the aggregate would be Sample 1 from Chain 100, Sample 101 of the aggregate would be Sample 2 from Chain 1, and so on. This approach has two benefits:

- Different chains should wind up in different probability regions, mitigating the local limitations of the method

- The multiple chains can be run simultaneously, reducing the time to attain results

Figure 8.33 shows the convergence in total variation norm resulting this aggregation method applied to 100 separate pCN chains, compared to an HMC chain with the same total runtime. $^{3}$ The observed convergence is similar to HMC or single-chain pCN for some components, but much faster for the components $v_{2}, v_{4}$, and $v_{8}$, which have multiple distinct

\footnotetext{
${ }^{3}$ The $\mathrm{pCN}$ chains used in this test case were different from those used to estimate the true posterior in Section 8.3.1.
} 
probability masses in the posterior. This is because some chains fall into and explore each probability well, minimizing the need for a single chain to jump between wells.

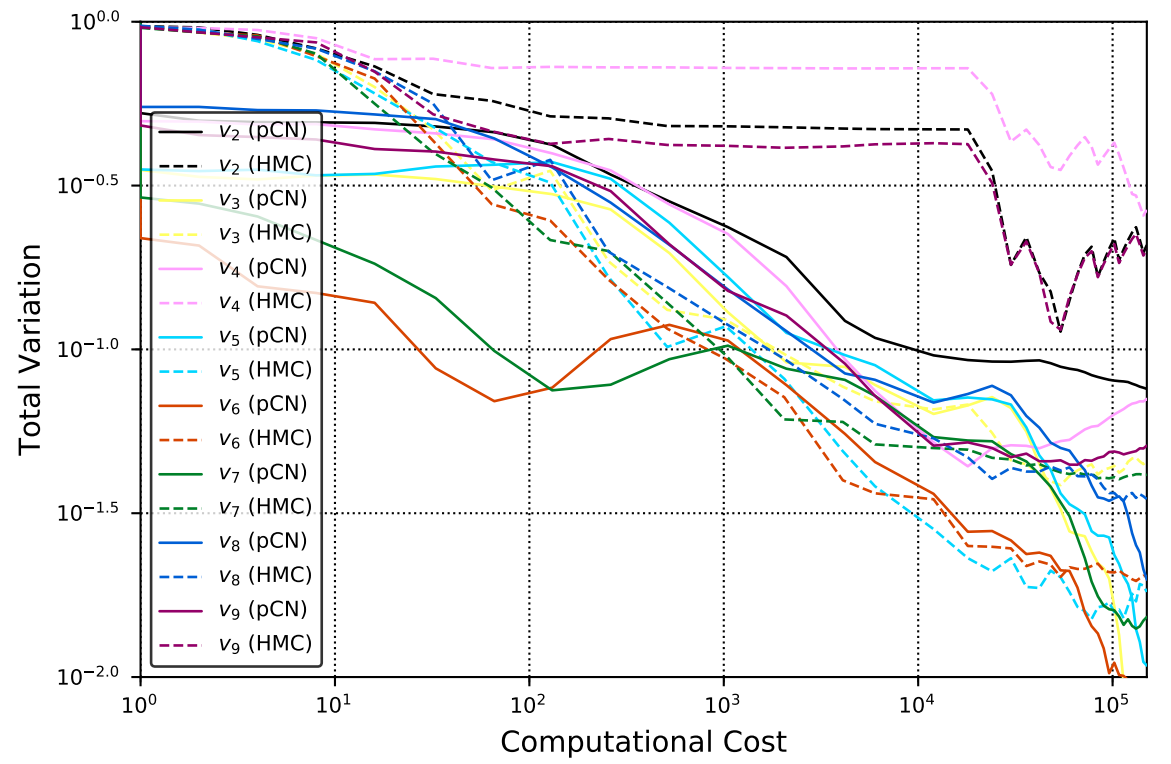

Figure 8.33: Total variation norm between computed and "true" marginal probability density function for $v_{2}, \ldots, v_{9}$ across $100 \mathrm{pCN}$ chains and $1 \mathrm{HMC}$ chain, by computational cost (1 computational unit $=1 \mathrm{HMC}$ sample on one computer). 


\subsubsection{Extension to Higher Dimensions via Particle Method}

In this example, we repeat Example 2 (Section 8.3) in higherdimensions to show the promise of the particle method described in Chapter 7. We therefore relax the previous assumption that the background flow has wave numbers with $\|\mathbf{k}\|_{2} \leq 8$ (197 total basis functions); here we allow $\|\mathbf{k}\|_{2} \leq 32$ (3,209 basis functions). When computed using the Galerkin reference method, the 16-fold increase in the dimension of $\mathbf{v}$ yielded an increase of over 300 in the computational cost of each sample, making it computationally intractable to generate enough samples to resolve the complex structure of the posterior distri-

\begin{tabular}{|c|c|}
\hline Method & $\frac{T_{2}}{T_{1}}$ \\
\hline \hline Reference & 302.3 \\
\hline Particle (CPU) & 35.2 \\
\hline Particle (GPU) & 8.9 \\
\hline
\end{tabular}

Table 8.7: Time to compute $\mathcal{G}$ for 197 basis functions $\left(T_{1}\right)$ vs. 3,209 basis functions $\left(T_{2}\right)$, by Method. bution. By contrast, the computational cost of the GPU-based particle method only increased by a factor of 9 , as some of the linear computational cost (7.2) was absorbed by the parallelism in the GPU. Table 8.7 compares the cost increase of the reference and particle methods for $\|\mathbf{k}\|_{2} \leq 8$ (197 basis functions, $\left.T_{1}\right)$ vs. $\|\mathbf{k}\|_{2} \leq 32$ (3,209 basis functions, $\left.T_{2}\right)$.

To approximate the posterior, we generated 2.5 million samples via 100 separate 25,000sample chains. Figure 8.34 shows the resulting posterior marginal distributions and Figure 8.35 shows the resulting two-dimensional posterior histograms. When compared with Figure 8.4 and Figure 8.5, respectively, we see that the posterior shows additional regions of probability mass - see, for example, the peaks for $v_{8} \approx \pm 1.5$. This indicates that the restriction of $\mathbf{v}$ to Fourier modes with $\|\mathbf{k}\| \leq 8$ caused some possible candidate vector fields to be missed or de-emphasized. 


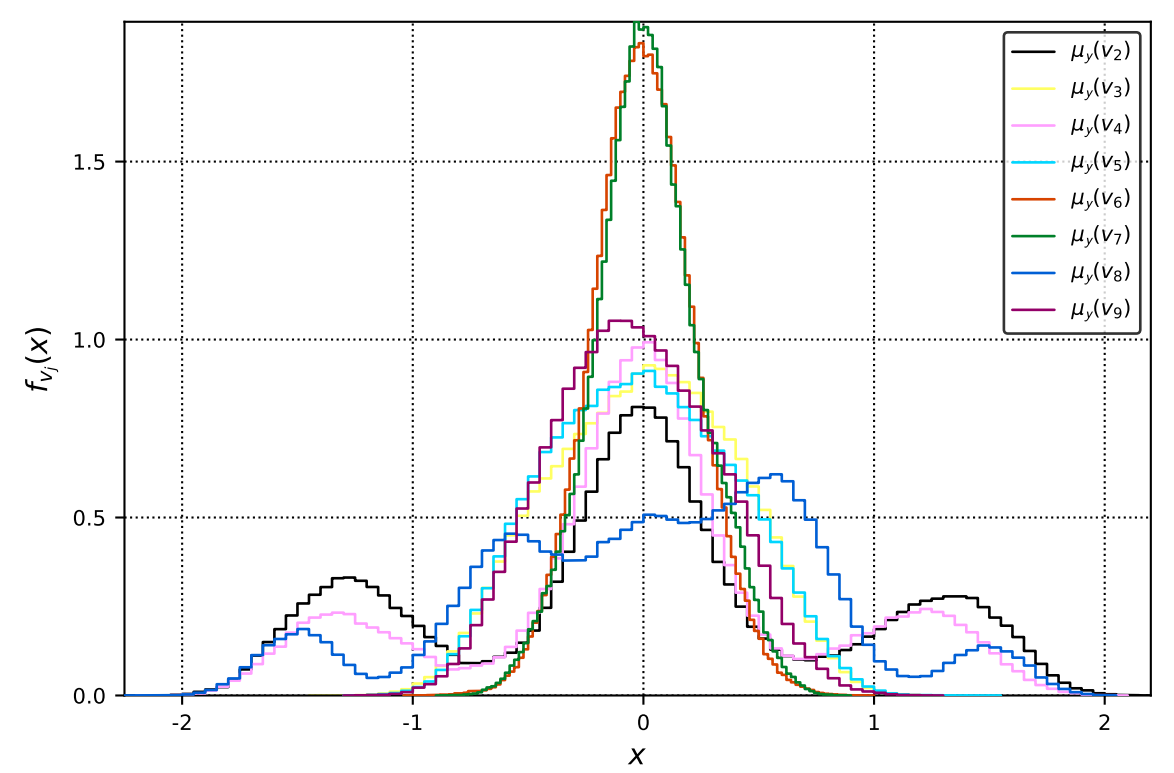

Figure 8.34: Posterior marginal distributions for the first eight components of $\mathbf{v}$. 


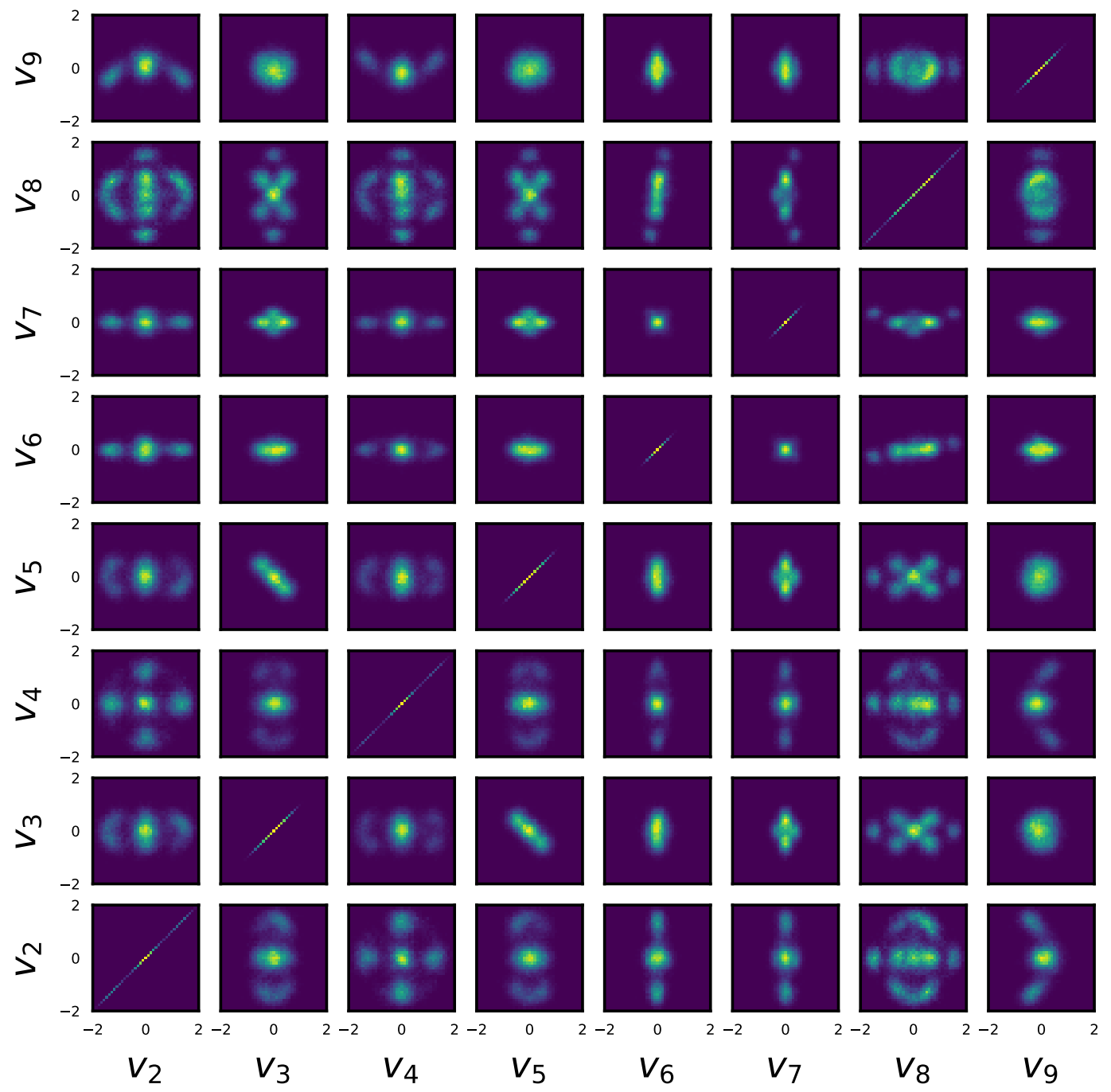

Figure 8.35: Posterior two-dimensional histograms for the first eight components of $\mathbf{v}$. 


\subsection{Summary of Results of Numerical Experiments}

To summarize the results of Examples 1-2, we see trade-offs between the MCMC methods:

- The independence sampler (IS) failed to provide an accurate approximation in either of the two examples that we tested, as the posterior was different enough from the prior that the prior proved to be a poor proposal distribution.

- pCN allows the practitioner an inexpensive method to generate samples and explore local regions of a probability measure. It also includes a parameter $\beta$ that can be tuned to the problem. For posterior distributions with simple structure, as in Example 1, this may be sufficient to achieve near-HMC convergence in a single MCMC chain, especially when compared on an equal run-time basis.

- MALA provided similar performance to $\mathrm{pCN}$ for the two examples that we describe, despite requiring much more time for computation (and code development) because of the need to compute $D \Phi$. The step size $h$ for MALA had to be very small for both Example 1 and Example 2 to keep the acceptance rate usable. This meant that consecutive samples tended to be as correlated with each other as for pCN.

- HMC samples are more computationally expensive to generate; for the HMC test cases reported here, each HMC sample took 39-125 times as much time as one pCN sample, though in general this ratio will be dictated by the cost of the gradient computation and the choice of number of integration steps $I=\frac{\tau}{\epsilon}$. However, HMC allows a practitioner to generate samples that approximate even complicated posteriors in a single chain. HMC uniformly produced the best convergence for observables and measures on a per-sample basis of any of the methods tested for both Example 1 and Example 2.

- Much of the difference between convergence of pCN and HMC may be eliminated when 
the methods are compared on the basis of runtime (rather than number of samples). Equal runtime results were similar for $\mathrm{pCN}$ and $\mathrm{HMC}$ in both Example 1 and Example 2. However, jumps between masses of probability in Example 2 were still significantly less frequent for $\mathrm{pCN}$ than $\mathrm{HMC}$, even when weighted by runtime. This may leave convergence of pCN more subject to random fluctuations than HMC.

- The weaknesses of pCN for complicated posteriors may be overcome in some cases by combining the results of an ensemble of different pCN chains. The different chains may wind up in different regions of the posterior and as an ensemble may allow resolution of multiple probability masses. For the example in this section, combining multiple pCN chains offered substantially better resolution of the posterior measure when compared with HMC using the same computational resources.

- Implementation of MALA and HMC is much more involved than IS or pCN. With a working PDE solver, pCN can be implemented in a matter of minutes or hours. Developing a gradient solver and implementing the HMC leap frog integration (and debugging both) can require on the order of days, weeks, or even months of development time. The implementation complexity is related to the complexity of the PDE solver. Debugging any of these methods is complicated by the randomness inherent in a given run and by the Metropolis-Hastings step, which can "correct for" improperlyimplemented proposals in a way that makes incorrect results look plausible.

We end by noting that in this case we have investigated only one possible failure mode for MCMC algorithms (a multiple-hump posterior). It is possible that there are other failure cases that will be overcome by HMC but not by combining multiple pCN chains. 


\section{Chapter 9}

\section{Conclusions and Future Work}

In this work we considered the inverse problem of estimating a background fluid flow $\mathbf{v}$ from noisy observations of a pollutant or other solute $\theta$ advecting and diffusing within the fluid. After showing that the traditional inverse problem was ill-posed - that is, may have many

or no solution(s) - we adopted a Bayesian approach, in which the problem is regularized by introducing statistical models of the fluid flow and observational noise.

In Chapter 4, we showed that, under certain regularity (smoothness) assumptions on the true background flow and compatibility assumptions on the prior measure, the true background flow can be recovered almost surely from point observations of the solute concentration, as the number of such observations grows infinitely large. We also demonstrated cases in which the assumptions were violated and convergence would not occur due to symmetries or translational invariances in the problem.

In Chapter 7, we introduced a particle method for computing point observations of $\theta$ from $\mathbf{v}$. The method leverages the nature of the inverse problem to bypass computation of the entire $\theta$ field, computing values only where they are needed. The method offers the potential to produce large speedup over traditional "compute, then observe" methods, especially for problems with high-dimensional $\mathbf{v}$ but low-dimensional observations.

In Chapter 8, we introduced two example problems with very different posterior structure; Section 8.2 produced a nearly-Gaussian posterior, while Section 8.3 yielded a highly complex 
posterior with several disparate masses of probability. We used these examples to conduct an in-depth benchmark of the applicability and convergence of the Independence, pCN, MALA, and HMC MCMC methods. The results indicated that HMC is uniformly superior on a per-sample basis. However, the computational cost can be much higher than for simpler methods so that, when compared on a computational cost basis, pCN and HMC exhibited largely similar convergence.

The parameter estimation problem provides a number of possible avenues for future research. The consistency argument in Chapter 4 could be generalized to a broader set of inverse problems; it also seems likely that some of the assumptions on $\mu_{0}$ and $\theta_{0}$ could be streamlined. The particle method in Chapter 7 could be applied to additional sample problems, such as those with a highly turbulent velocity field, or to inverse problems other than the one that we have considered here. The example problem in Section 8.3 exhibits a number of interesting features, which could be examined in more depth. More generally, the Bayesian method offers an extremely useful approach to computational inverse problems that can be applied to a number of application areas, and may show particular promise where observations are sufficiently noisy that they are difficult to interpret without a formalized, statistical framework. 


\section{Bibliography}

[1] Oriole Park at Camden Yards. URL https://en.wikipedia.org/wiki/Oriole_Park_ at_Camden_Yards.

[2] Volkan Akçelik, George Biros, Omar Ghattas, Kevin R Long, and Bart van Bloemen Waanders. A variational finite element method for source inversion for convectivediffusive transport. Finite Elements in Analysis and Design, 39(8):683-705, 2003.

[3] Moritz Allmaras, Wolfgang Bangerth, Jean Marie Linhart, Javier Polanco, Fang Wang, Kainan Wang, Jennifer Webster, and Sarah Zedler. Estimating parameters in physical models through Bayesian inversion: A complete example. SIAM Review, 55(1):149-167, 2013.

[4] Richard Askey and James Arthur Wilson. Some basic hypergeometric orthogonal polynomials that generalize Jacobi polynomials, volume 319. American Mathematical Society, 1985.

[5] Marco Baiesi and Christian Maes. Enstrophy dissipation in two-dimensional turbulence. Physical Review E, 72(5):056314, 2005.

[6] George K Batchelor. Computation of the energy spectrum in homogeneous twodimensional turbulence. The Physics of Fluids, 12(12):II-233, 1969.

[7] Thomas Bayes. An essay towards solving a problem in the doctrine of chances. Philosophical Transactions of the Royal Society of London Series I, 53:370-418, 1763.

[8] Alexandros Beskos and Andrew Stuart. MCMC methods for sampling function space. In 
Invited Lectures, Sixth International Congress on Industrial and Applied Mathematics, ICIAM07, Editors Rolf Jeltsch and Gerhard Wanner, pages 337-364, 2009.

[9] Alexandros Beskos, Gareth Roberts, Andrew Stuart, and Jochen Voss. MCMC methods for diffusion bridges. Stochastics and Dynamics, 8(03):319-350, 2008.

[10] Alexandros Beskos, Gareth Roberts, Andrew Stuart, et al. Optimal scalings for local Metropolis-Hastings chains on nonproduct targets in high dimensions. The Annals of Applied Probability, 19(3):863-898, 2009.

[11] Alexandros Beskos, Frank J Pinski, Jesús Marıa Sanz-Serna, and Andrew M Stuart. Hybrid Monte Carlo on Hilbert spaces. Stochastic Processes and their Applications, 121 (10):2201-2230, 2011.

[12] Alexandros Beskos, Mark Girolami, Shiwei Lan, Patrick E Farrell, and Andrew M Stuart. Geometric MCMC for infinite-dimensional inverse problems. Journal of Computational Physics, 335:327-351, 2017.

[13] Jeff Bezanson, Alan Edelman, Stefan Karpinski, and Viral B Shah. Julia: A fresh approach to numerical computing. SIAM review, 59(1):65-98, 2017.

[14] Nawaf Bou-Rabee, Martin Hairer, and Eric Vanden-Eijnden. Non-asymptotic mixing of the MALA algorithm. IMA Journal of Numerical Analysis, 33, 082010.

[15] Tan Bui-Thanh and Mark Girolami. Solving large-scale PDE-constrained Bayesian inverse problems with Riemann manifold Hamiltonian Monte Carlo. Inverse Problems, 30(11):114014, 2014.

[16] Tan Bui-Thanh, Omar Ghattas, James Martin, and Georg Stadler. A computational framework for infinite-dimensional Bayesian inverse problems Part I: The linearized case, 
with application to global seismic inversion. SIAM Journal on Scientific Computing, 35 (6):A2494-A2523, 2013.

[17] Bob Carpenter, Andrew Gelman, Matt Hoffman, Daniel Lee, Ben Goodrich, Michael Betancourt, Michael A Brubaker, Jiqiang Guo, Peter Li, and Allen Riddell. Stan: A probabilistic programming language. Journal of Statistical Software, 20:1-37, 2016.

[18] Shiyi Chen and Robert H Kraichnan. Simulations of a randomly advected passive scalar field. Physics of Fluids (1994), 10(11), 1998.

[19] Alexandre Joel Chorin. Numerical study of slightly viscous flow. Journal of Fluid Mechanics, 57(4):785-796, 1973. doi: 10.1017/S0022112073002016.

[20] Ramon Codina. On stabilized finite element methods for linear systems of convectiondiffusion-reaction equations. Computer Methods in Applied Mechanics and Engineering, 188(1):61-82, 2000.

[21] Simon L Cotter, Gareth O Roberts, Andrew M Stuart, and David White. MCMC methods for functions: modifying old algorithms to make them faster. Statistical Science, 28 (3):424-446, 2013.

[22] Edward Lansing Cussler. Diffusion: mass transfer in fluid systems. Cambridge University Press, 2009.

[23] Giuseppe Da Prato and Jerzy Zabczyk. Stochastic equations in infinite dimensions, volume 152. Cambridge University Press, 2014.

[24] Masoumeh Dashti and Andrew M Stuart. The Bayesian approach to inverse problems. Handbook of Uncertainty Quantification, pages 311-428, 2017. 
[25] Pierre Degond and S Mas-Gallic. The weighted particle method for convection-diffusion equations. I. the case of an isotropic viscosity. Mathematics of computation, 53(188): 485-507, 1989.

[26] Persi Diaconis and David Freedman. On the consistency of Bayes estimates. The Annals of Statistics, pages 1-26, 1986.

[27] Joseph L Doob. Application of the theory of martingales. Le calcul des probabilites et ses applications, pages 23-27, 1949.

[28] Arnaud Doucet, Simon J Godsill, and Christian P Robert. Marginal maximum a posteriori estimation using Markov chain Monte Carlo. Statistics and Computing, 12(1): $77-84,2002$.

[29] Simon Duane, Anthony D Kennedy, Brian J Pendleton, and Duncan Roweth. Hybrid Monte Carlo. Physics letters B, 195(2):216-222, 1987.

[30] AW Eggels, RPJ Kunnen, Barry Koren, and AS Tijsseling. Infotaxis in a turbulent 3D channel flow. Journal of Computational and Applied Mathematics, 310:44-58, 2017.

[31] Lawrence C. Evans. Partial Differential Equations. Graduate studies in mathematics. American Mathematical Society, second edition, 2010. ISBN 9780821807729.

[32] David A Fournier, Hans J Skaug, Johnoel Ancheta, James Ianelli, Arni Magnusson, Mark N Maunder, Anders Nielsen, and John Sibert. AD Model Builder: using automatic differentiation for statistical inference of highly parameterized complex nonlinear models. Optimization Methods and Software, 27(2):233-249, 2012.

[33] David A Freedman. On the asymptotic behavior of Bayes' estimates in the discrete case. The Annals of Mathematical Statistics, pages 1386-1403, 1963. 
[34] Andrew Gelman, John B Carlin, Hal S Stern, David B Dunson, Aki Vehtari, and Donald B Rubin. Bayesian Data Analysis. CRC Press, third edition, 2014.

[35] Roger G Ghanem and Pol D Spanos. Stochastic finite elements: a spectral approach. Courier Corporation, 2003.

[36] Mark Girolami and Ben Calderhead. Riemann manifold Langevin and Hamiltonian Monte Carlo methods. Journal of the Royal Statistical Society: Series B (Statistical Methodology), 73(2):123-214, 2011.

[37] Ulf Grenander and Michael I. Miller. Representations of knowledge in complex systems. Journal of the Royal Statistical Society. Series B (Methodological), 56(4):549-603, 1994. ISSN 00359246. URL http://www. jstor.org/stable/2346184.

[38] Martin Hairer and Jonathan C Mattingly. Yet another look at Harris' ergodic theorem for Markov chains. In Seminar on Stochastic Analysis, Random Fields and Applications VI, pages 109-117. Springer, 2011.

[39] Martin Hairer, Jonathan C Mattingly, and Michael Scheutzow. Asymptotic coupling and a general form of Harris' theorem with applications to stochastic delay equations. Probability Theory and Related Fields, 149(1-2):223-259, 2011.

[40] Martin Hairer, Andrew M Stuart, Sebastian J Vollmer, et al. Spectral gaps for a Metropolis-Hastings algorithm in infinite dimensions. The Annals of Applied Probability, 24(6):2455-2490, 2014.

[41] W Keith Hastings. Monte Carlo sampling methods using Markov chains and their applications. Biometrika, 57(1):97-109, 1970.

[42] Jan S Hesthaven and Tim Warburton. Nodal discontinuous Galerkin methods: algorithms, analysis, and applications. Springer Science \& Business Media, 2007. 
[43] Desmond J Higham. An algorithmic introduction to numerical simulation of stochastic differential equations. SIAM review, 43(3):525-546, 2001.

[44] Michael Hinze, René Pinnau, Michael Ulbrich, and Stefan Ulbrich. Optimization with PDE constraints, volume 23 of Mathematical Modelling: Theory and Applications. Springer Science \& Business Media, 2009.

[45] Willem Hundsdorfer and Jan G Verwer. Numerical solution of time-dependent advectiondiffusion-reaction equations, volume 33. Springer Science \& Business Media, 2013.

[46] Jari Kaipio and Erkki Somersalo. Statistical and computational inverse problems, volume 160 of Applied Mathematical Sciences. Springer Science \& Business Media, 2005.

[47] Peter E Kloeden and Eckhard Platen. Numerical Solution of Stochastic Differential Equations. Springer, 1999.

[48] Andrei Nikolaevich Kolmogorov. On the analytic methods of probability theory. Uspekhi matematicheskikh nauk, 5:5-41, 1938.

[49] Robert H Kraichnan. Inertial ranges in two-dimensional turbulence. The Physics of Fluids, 10(7):1417-1423, 1967.

[50] Robert H Kraichnan. Small-scale structure of a scalar field convected by turbulence. The Physics of Fluids, 11(5):945-953, 1968.

[51] Robert H Kraichnan. Stochastic modeling of isotropic turbulence. In New Perspectives in Turbulence, pages 1-54. Springer, 1991.

[52] Antti Kupiainen. Statistical theories of turbulence, 2000. Lectures given at the workshop Random media 2000. 
[53] Pierre-Simon Laplace. Mémoire sur les approximations des formules qui sont fonctions de très grands nombres et sur leur applications aux probabilités. Memoires de l'Academie des Sciences de Paris, 1810.

[54] Lucien Le Cam and Grace Lo Yang. Asymptotics in Statistics: Some Basic Concepts. Springer Science \& Business Media, 2000.

[55] Lucien LeCam. On some asymptotic properties of maximum likelihood estimates and related Bayes estimates. Univ. California Pub. Statist., 1:277-330, 1953.

[56] Andrew J Majda and Andrea L Bertozzi. Vorticity and incompressible flow, volume 27. Cambridge University Press, 2002.

[57] Youssef M Marzouk and Habib N Najm. Dimensionality reduction and polynomial chaos acceleration of Bayesian inference in inverse problems. Journal of Computational Physics, 228(6):1862-1902, 2009.

[58] Youssef M Marzouk, Habib N Najm, and Larry A Rahn. Stochastic spectral methods for efficient Bayesian solution of inverse problems. Journal of Computational Physics, 224(2):560-586, 2007.

[59] Jonathan C Mattingly, Natesh S Pillai, Andrew M Stuart, et al. Diffusion limits of the random walk metropolis algorithm in high dimensions. The Annals of Applied Probability, 22(3):881-930, 2012.

[60] Nicholas Metropolis, Arianna W Rosenbluth, Marshall N Rosenbluth, Augusta H Teller, and Edward Teller. Equation of state calculations by fast computing machines. The Journal of Chemical Physics, 21(6):1087-1092, 1953.

[61] Keith W Morton. Numerical solution of convection-diffusion problems. Chapman \& Hall, 1996. 
[62] MK Mudunuru and KB Nakshatrala. On enforcing maximum principles and achieving element-wise species balance for advection-diffusion-reaction equations under the finite element method. Journal of Computational Physics, 305:448-493, 2016.

[63] Habib N Najm. Uncertainty quantification and polynomial chaos techniques in computational fluid dynamics. Annual Review of Fluid Mechanics, 41:35-52, 2009.

[64] Radford M Neal. Bayesian Learning for Neural Networks. Springer-Verlag New York, Inc., 1996.

[65] Radford M Neal et al. MCMC using Hamiltonian dynamics. Handbook of Markov Chain Monte Carlo, 2:113-162, 2011.

[66] Whitney K Newey and Daniel McFadden. Large sample estimation and hypothesis testing. Handbook of econometrics, 4:2111-2245, 1994.

[67] NVIDIA. NVIDIA CUDA C programming guide. NVIDIA Corporation, 2018.

[68] Bernt Øksendal. Stochastic Differential Equations. An Introduction with Applications, volume 5. Springer, New York, 6th edition, 2013.

[69] Noemi Petra, James Martin, Georg Stadler, and Omar Ghattas. A computational framework for infinite-dimensional Bayesian inverse problems, Part II: Stochastic Newton MCMC with application to ice sheet flow inverse problems. SIAM Journal on Scientific Computing, 36(4):A1525-A1555, 2014.

[70] Natesh S Pillai, Andrew M Stuart, Alexandre H Thiéry, et al. Optimal scaling and diffusion limits for the Langevin algorithm in high dimensions. The Annals of Applied Probability, 22(6):2320-2356, 2012.

[71] Gareth O Roberts and Jeffrey S Rosenthal. Optimal scaling for various MetropolisHastings algorithms. Statistical Science, 16(4):351-367, 2001. 
[72] Gareth O Roberts and Richard L Tweedie. Exponential convergence of Langevin distributions and their discrete approximations. Bernoulli, 2(4):341-363, 1996.

[73] Gareth O Roberts, Andrew Gelman, Walter R Gilks, et al. Weak convergence and optimal scaling of random walk Metropolis algorithms. The annals of applied probability, 7(1):110-120, 1997.

[74] James C Robinson. Infinite-dimensional Dynamical Systems: An introduction to dissipative parabolic PDEs and the theory of global attractors, volume 28. Cambridge University Press, 2001.

[75] FW Scholz. Maximum likelihood estimation. Encyclopedia of statistical sciences, 1985.

[76] Lorraine Schwartz. On Bayes procedures. Zeitschrift für Wahrscheinlichkeitstheorie und verwandte Gebiete, 4(1):10-26, 1965.

[77] Xiaotong Shen and Larry Wasserman. Rates of convergence of posterior distributions. Annals of Statistics, pages 687-714, 2001.

[78] Boris I Shraiman and Eric D Siggia. Scalar turbulence. Nature, 405(6787):639-646, 2000.

[79] KR Sreenivasan. On local isotropy of passive scalars in turbulent shear flows. Proc. $R$. Soc. Lond. A, 434(1890):165-182, 1991.

[80] Andrew M Stuart. Inverse problems: a Bayesian perspective. Acta Numerica, 19: $451-559,2010$.

[81] Martin Stynes. Numerical methods for convection-diffusion problems or the 30 years war. arXiv preprint arXiv:1306.5172, 2013. 
[82] Luke Tierney. A note on Metropolis-Hastings kernels for general state spaces. Annals of Applied Probability, pages 1-9, 1998.

[83] Sebastian J Vollmer. Dimension-independent MCMC sampling for inverse problems with non-Gaussian priors. SIAM/ASA Journal on Uncertainty Quantification, 3(1): 535-561, 2015.

[84] Z Warhaft. Passive scalars in turbulent flows. Annual Review of Fluid Mechanics, 32 (1):203-240, 2000.

[85] Star Wars. Darth Vader's TIE fighter. URL https://www.starwars.com/databank/ darth-vader-s-tie-fighter.

[86] Norbert Wiener. The homogeneous chaos. American Journal of Mathematics, 60(4): 897-936, 1938.

[87] Dongbin Xiu and George Em Karniadakis. The Wiener-Askey polynomial chaos for stochastic differential equations. SIAM Journal on Scientific Computing, 24(2):619644, 2002.

[88] Dongbin Xiu and George Em Karniadakis. A new stochastic approach to transient heat conduction modeling with uncertainty. International Journal of Heat and Mass Transfer, 46(24):4681-4693, 2003.

[89] Sergiy Zhuk, Tigran T Tchrakian, Stephen Moore, Rodrigo Ordóñez-Hurtado, and Robert Shorten. On source-term parameter estimation for linear advection-diffusion equations with uncertain coefficients. SIAM Journal on Scientific Computing, 38(4): A2334-A2356, 2016. 